JOURNAL OF THE

AMERICAN MATHEMATICAL SOCIETY

Volume 15, Number 4, Pages 929-978

S 0894-0347(02)00393-4

Article electronically published on June 21, 2002

\title{
FINITE QUOTIENTS OF THE MULTIPLICATIVE GROUP OF A FINITE DIMENSIONAL DIVISION ALGEBRA ARE SOLVABLE
}

\author{
ANDREI S. RAPINCHUK, YOAV SEGEV, AND GARY M. SEITZ
}

\section{INTRODUCTION}

The purpose of this paper is to prove the following.

Main Theorem. Let $D$ be a finite dimensional division algebra. Then any finite quotient of the multiplicative group $D^{\times}$is solvable.

This result is a culmination of research done in the last several years in order to restrict the structure of finite quotients of $D^{\times}$. One of the principal motivations for this research was the work done on the Margulis-Platonov conjecture (MP) for anisotropic algebraic groups of inner type $A_{n}$ over global fields; these are precisely the groups of the form $\mathbf{S L}_{1, D}$ associated with the group $S L(1, D)$ of elements having reduced norm 1 in some finite dimensional division algebra $D$. Referring the reader to Ch. IX in [15] and Appendix A in 20] for a discussion of (MP), we only point out here that (MP) for $\mathbf{S L}_{1, D}$ was reduced in [19] to the following statement which is meaningful for division algebras over arbitrary fields: $D^{\times}$does not have quotients that are nonabelian finite simple groups. This fact was verified in 19] for division algebras of degree 2 and 3 , and stated for arbitrary finite dimensional division algebras as a conjecture. The affirmative resolution of this conjecture was obtained in [23] and [26]. In 23] techniques were developed for analyzing finite quotients $D^{\times} / N$ using the commuting graph 11 of $D^{\times} / N$, and some of the constructions in [23] were basically equivalent to proving the openness of $N$ with respect to a nontrivial valuation of $D$ under the assumption that the commuting graph of $D^{\times} / N$ either has diameter $\geq 5$ or is "balanced". Valuations were explicitly used for the first

Received by the editors February 28, 2001 and, in revised form, January 24, 2002.

1991 Mathematics Subject Classification. Primary 16K20, 16U60; Secondary 20G15, 05C25.

Key words and phrases. Division algebra, multiplicative group, finite homomorphic images, valuations.

The first author was partially supported by grants from the NSF and by BSF grant no. 9700042 .

The second author was partially supported by BSF grant no. 97-00042. Portions of this work were written while the author visited the Forschungsinstitut für Mathematik ETH, Zurich, in the summer of 2000, and the author gratefully acknowledges the hospitality and support.

The third author was partially supported by grants from the NSF and by BSF grant no. 9700042 .

${ }^{1}$ We recall that the commuting graph $\Delta_{H}$ of a finite group $H$ is the graph whose vertex set is $H \backslash\{1\}$ and whose edges are pairs of distinct commuting elements; of course, $\Delta_{H}$ has a natural distance function $d_{H}$ which, in particular, allows one to talk about the diameter of $\Delta_{H}$, denoted $\operatorname{diam}\left(\Delta_{H}\right)$. 
time in 20] where the following "openness theorem" was obtained: if the diameter of the commuting graph of $D^{\times} / N$ is $\geq 4$, then $N$ is open in $D^{\times}$with respect to a nontrivial valuation of $D$ (Theorem 1 in [20]).

Let us indicate now how the openness of $N$ can be used to restrict the structure of the finite quotient $D^{\times} / N$ (and eventually to eliminate nonsolvable finite groups as quotients of $D^{\times}$). First, since $D$ is finite dimensional, Wedderburn's theorem allows us to assume that $K$ is infinite (else $D$ is finite and hence commutative). Now, in $\S 8$ of [20], the following "nonexistence" result was actually established (it was however stated in a slightly less general form).

Nonexistence Theorem. Let $\mathcal{G}$ be a class of finite groups. Call a member $G \in \mathcal{G}$ minimal if no proper quotient of $G$ belongs to $\mathcal{G}$. Assume that

(1) the members of $\mathcal{G}$ are not solvable;

(2) if $G \in \mathcal{G}$ and $M \triangleleft G$ with $G / M$ solvable, then $M \in \mathcal{G}$;

(3) if $G \in \mathcal{G}$ and $M \triangleleft G$ is a solvable normal subgroup, then $G / M \in \mathcal{G}$;

(4) if $G \in \mathcal{G}$ is a minimal member, then given a finite dimensional division algebra $D$ over a finitely generated field and a surjective homomorphism $\phi: D^{\times} \rightarrow G$, the kernel $\operatorname{Ker} \phi$ is open in $D^{\times}$with respect to a nontrivial height one valuation of $D$.

Then no member of $\mathcal{G}$ can be a quotient of the multiplicative group of any finite dimensional division algebra.

We refer the reader to the beginning of $\S 2$ for the definition of a (height one) valuation and for the notion of openness. We note that a sketch of the proof of the Nonexistence Theorem is given at the beginning of $\S 6$.

In view of the Nonexistence Theorem, the above mentioned "openness theorem" (Theorem 1 in [20]) implies that if $\mathcal{G}$ is a class of finite groups satisfying (1) - (3) of the Nonexistence Theorem, and whose minimal members $G$ have the property that $\operatorname{diam}\left(\Delta_{G}\right) \geq 4$, then no member of $\mathcal{G}$ is a quotient of any $D^{\times}$(cf. "Nonexistence Theorem at Diameter $\geq 4$ " in 20]). This theorem applies to some important classes. For example, since by $[26$ the commuting graph of nonabelian finite simple groups has diameter $\geq 4$, it applies to the class $\mathcal{G}$ of nonabelian finite simple groups, eliminating them as possible quotients of $D^{\times}$. However, Theorem 1 of 20] falls short of extending the Nonexistence Theorem to the class $\mathcal{G}=\mathcal{N} \mathcal{S}$ of all finite nonsolvable groups. The reason is that the diameter of the commuting graph of minimal nonsolvable groups (i.e. nonsolvable finite groups all of whose proper quotients are solvable; these are called MNS groups in the sequel) may be equal to 3 (and is always $\geq 3$; cf. 25). At the same time, there are examples (cf. 8.4 in 20]) where $\operatorname{diam}\left(\Delta_{D \times / N}\right)=3$, but $N$ is not open with respect to any nontrivial valuation of $D$. So the mere consideration of diameters of the commuting graphs does not lead to an openness result sufficient to apply the Nonexistence Theorem to the class $\mathcal{N} S$. The success in the current paper is achieved through the use of a new, more refined condition (see Property $\left(3 \frac{1}{2}\right)$ below), which, on the one hand, holds for all MNS groups and, on the other hand, is strong enough to prove an "openness theorem".

We pause to mention that the assertion of the Main Theorem was conjectured by Segev in [24]. This conjecture was formulated in view of the results in [23], [26] and also the results with L. Rowen [21] and [22] that finite quotients of $D^{\times}$, where $D$ is a division algebra of degree 3 or 5 , are solvable (the last two results were obtained using very different tools, e.g., "Wedderburn's factorization theorem"). 
Here is a precise formulation of the property of commuting graphs which is crucial to this work.

Property $\left(3 \frac{1}{2}\right)$. There are two elements $x, y \in H \backslash\{1\}$ such that for all $a, b \in$ $H \backslash\{1\}$ satisfying $[x, a]=[y, b]=1$, there exists $h \in H$ with the property $d_{H}\left(x^{h}, y\right) \geq$ 3 and $\left[a^{h}, b\right] \neq 1$, where as usual $x^{h}=h^{-1} x h$ and $[x, a]=x^{-1} a^{-1} x a$.

Clearly, Property $\left(3 \frac{1}{2}\right)$ implies that $\operatorname{diam}\left(\Delta_{H}\right) \geq 3$. On the other hand, any elements $x, y \in H \backslash\{1\}$ with $d_{H}(x, y) \geq 4$ will satisfy Property $\left(3 \frac{1}{2}\right)$ as one can take $h=1$. Thus, Property $\left(3 \frac{1}{2}\right)$ is stronger than the condition $\operatorname{diam}\left(\Delta_{H}\right) \geq 3$, but weaker than the condition $\operatorname{diam}\left(\Delta_{H}\right) \geq 4$. Now we are in a position to formulate the following (cf. Theorem 6.1).

Theorem 1. Let $D$ be a finite dimensional division algebra over a finitely generated infinite field, and let $N \subseteq D^{\times}$be a normal subgroup of finite index. If $H=D^{\times} / N$ satisfies Property $\left(3 \frac{1}{2}\right)$, then $N$ is open in $D^{\times}$with respect to a nontrivial height one valuation of $D$.

As Property $\left(3 \frac{1}{2}\right)$ follows from the condition $\operatorname{diam}\left(\Delta_{H}\right) \geq 4$, this theorem contains Theorem 1 of $\left[20\right.$, on the openness of $N$ if $\operatorname{diam}\left(\Delta_{D^{\times} / N}\right) \geq 4$. The following theorem warrants application of Theorem 1 in the case where $D^{\times} / N$ is a minimal nonsolvable group.

Theorem 2. Let $H$ be a minimal finite nonsolvable group. Then $H$ has Property $\left(3 \frac{1}{2}\right)$.

The proof of Theorem 2 is carried out in $\S 7$; it depends on the classification of finite simple groups and uses detailed information about their structure. As we explained above, Theorems 1 and 2, in conjunction with the Nonexistence Theorem, yield the Main Theorem.

It should be pointed out that our Main Theorem has important implications even in the case where $D$ is a finite dimensional central division algebra over a global field $K$. In particular, combined with a theorem due to Margulis and Prasad (see [13], [17] and $\S 6$ ), it implies that all finite quotients of $S L(1, D)$ are solvable (Corollary 6.3). The latter fact enables one to give a short proof of the Margulis-Platonov conjecture (MP) for the group $\mathbf{S L}_{1, D}$, which in this set-up claims the openness of an arbitrary noncentral normal subgroup $M$ of $S L(1, D)$ in the topology defined by the (finite) set $T$ of all nontrivial valuations of $D$. Indeed, by the above-mentioned theorem of Margulis and Prasad, $M$ has finite index in $S L(1, D)$. By our Corollary 6.3, the quotient $S L(1, D) / M$ is then solvable, so $M$ contains some term of the derived series of $S L(1, D)$. Furthermore, by a theorem of Raghunathan [18, all terms of the derived series are open with respect to the topology in question. The openness of $M$ follows, proving conjecture (MP) for the group $\mathbf{S L}_{1, D}$ (see Theorem 6.4). We observe that the original proof of (MP) for the group $\mathbf{S L}_{1, D}$, obtained in [23] and [26], used the reduction given in [19], which the above proof does not require.

We now briefly describe the methods employed in the proof of Theorem 1. First, we show in $\S \$ 2$ - 3 that a required valuation can be constructed given a homomorphism $\varphi: N \rightarrow \Gamma$ satisfying some special properties, where $\Gamma$ is a partially ordered group. A result of this kind was proved in [20] assuming that $\Gamma$ is totally ordered and $\varphi$ is a valuation-like map, i.e. there exists a nonnegative $\alpha \in \Gamma$, called a level 
of $\varphi$, such that

$$
N_{<-\alpha}+1 \subseteq N_{<-\alpha},
$$

where for $\gamma \in \Gamma$ we let $N_{<\gamma}:=\{n \in N \mid \varphi(n)<\gamma\}$. In order to prove Theorem 1 we need to deal with the situation where $\varphi$ still has a level, but the group $\Gamma$ is no longer guaranteed to be totally ordered (in this case we call $\varphi$ a leveled map). In $\S \S 2-3$ we define the notion of a valuation associated with a leveled map. We then single out a set of conditions on a given leveled map that ensure the existence of a valuation associated with it and the openness of $N$ with respect to this valuation. We then show in 5 how to construct a leveled map $\varphi: N \rightarrow \Gamma$ satisfying these conditions given that $D^{\times} / N$ has Property $\left(3 \frac{1}{2}\right)$ (without getting into technical details, we point out that the conditions include the requirements that $\Gamma_{K}:=\varphi\left(K^{\times}\right)$be a nontrivial totally ordered subgroup of $\Gamma$ and that $\varphi$ has a level in $\Gamma_{K}$; note that since $\operatorname{diam}\left(\Delta_{D^{\times} / N}\right) \geq 3$, we have $\left.K^{\times} \subseteq N\right)$. The argument in $\$ 5$ involves a new concept of strongly leveled maps, some properties of which are analyzed in 8 . We note that both the notion of a leveled map and of a strongly leveled map are closely related to condition (U3) of the U-hypothesis in $\S 3$ of 23 .

We conclude the introduction with two questions that naturally arise in the context of the investigation of the normal subgroup structure of algebraic groups over arbitrary fields. In the first question we ask what version of Theorem 1 can be proved under the mere assumption that the diameter of $\Delta_{D^{\times} / N}$ is $\geq 3$.

Question 1. Let $D$ be a finite dimensional division algebra over a finitely generated infinite field, and let $N \subseteq D^{\times}$be a normal subgroup of finite index. Does the fact that the commuting graph of $D^{\times} / N$ has diameter $\geq 3$ imply that $N$ is open in $D^{\times}$ with respect to a finite set $T$ of nontrivial height one valuations of $D$ ?

This question was first raised in [20], but still remains unresolved. We remark that the Nonexistence Theorem holds true even when in (4), Ker $\phi$ is required to be open with respect to a finite set $T$ of nontrivial height one valuations of $D$, but this more general version of the Nonexistence Theorem is not used in this paper (cf. Remark 8.3 in 20]). It follows that an affirmative answer to Question 1 would give an alternative proof of the Main Theorem which instead of the technically complicated Theorem 2 would rely only on the fact, proved in [25], that the diameter of the commuting graph of any MNS group is $\geq 3$. Other applications would include detailed information about possible finite quotients of $D^{\times}$which may eventually lead to some form of their classification (we recall that finite subgroups of $D^{\times}$were classified by Amitsur [1]).

The second question that came up in discussions of G. Prasad with Rapinchuk deals with extending our Main Theorem to other types of algebraic groups.

Question 2 (Prasad, Rapinchuk). Let $G$ be an absolutely simple algebraic group over an infinite field $K$. Is it true that all finite quotients of $G(K)$ are solvable?

We recall that if $G$ is $K$-isotropic, then the subgroup $G(K)^{+}$generated by the $K$-rational points of the unipotent radicals of $K$-defined parabolics does not have proper noncentral normal subgroups (Tits 31]), so any finite quotient of $G(K)$ is in fact a quotient of $W(G, K)=G(K) / G(K)^{+}$which was termed the Whitehead group of $G$ by Tits [32]. Since $W(G, K)$ is known to be abelian for most isotropic groups of classical types, this implies the affirmative answer to Question 2 in all these cases. The situation for $K$-anisotropic groups is different as very little is 
known about them when $K$ is a general field. However, some optimism regarding Question 2 is associated with the fact that results on the normal subgroup structure of the groups of type $A_{n}$ over global fields constitute a basis for consideration of other types (cf. [15], Ch. IX), and the Main Theorem strongly suggests the affirmative answer to Question 2 for anisotropic inner forms of type $A_{n}$.

We are grateful to Gopal Prasad for the inspiring interest he has shown in this work, for reading and listening to portions of this manuscript very carefully and for his helpful detailed remarks.

\section{The Existence of A VAluation Associated With A LEVELED MAP}

Throughout this paper, $D$ is a finite dimensional central division algebra over an infinite field $K$, and $N \subseteq D^{\times}$is a finite index subgroup such that $-1 \in N$. Recall that a valuation of $D$ is a group homomorphism $v: D^{\times} \rightarrow \tilde{\Gamma}$, from $D^{\times}$onto a linearly ordered group $\tilde{\Gamma}$ satisfying $v(x+y) \geq \min \{v(x), v(y)\}$ whenever $x+y \neq 0$. The group $\tilde{\Gamma}$ and the valuation $v$ are said to have height one if $\tilde{\Gamma}$ is isomorphic to a subgroup of the additive group $(\mathbb{R},+)$ of the reals. Throughout, $\Gamma$ denotes a partially ordered group 2 such that $\Gamma_{>0}:=\{\gamma \in \Gamma \mid \gamma>0\}$ is nonempty. We will consider surjective homomorphisms $\varphi: N \rightarrow \Gamma$.

Definitions 2.1. Let $\Gamma$ be a partially ordered group such that the set of positive elements of $\Gamma$ is nonempty. Let $\varphi: N \rightarrow \Gamma$ be a surjective homomorphism. Then

(1) We say that $\varphi$ is a leveled map if there exists a nonnegative $\alpha \in \Gamma$ (called a level of $\varphi$ ) such that

$$
N_{<-\alpha}+1 \subseteq N_{<-\alpha},
$$

where $N_{<-\alpha}=\{x \in N \mid \varphi(x)<-\alpha\}$. Note that we automatically have $N_{<-\alpha}$ $\neq \emptyset$.

(2) A valuation $v: D^{\times} \rightarrow \tilde{\Gamma}$ is associated with $\varphi$ if there exists a nontrivial homomorphism $\theta: \Gamma \rightarrow \tilde{\Gamma}$ of (partially) ordered groups such that the diagram

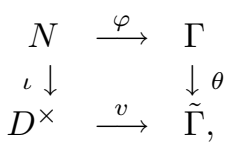

in which $\iota$ is the inclusion map, commutes.

We note that the notion of a leveled map extends the notion of a valuation-like map from 20 and will eventually lead to valuations. We fix the following notation.

Notation 2.2. Let $\Gamma$ be a nontrivial partially ordered group (not necessarily abelian but written additively!), and let $\varphi: N \rightarrow \Gamma$ be a (surjective) homomorphism (which will always be clear from the context).

(1) For $\beta \in \Gamma$, we let $\Gamma_{<\beta}$ (resp., $\Gamma_{\leq \beta}, \Gamma_{>\beta}$, etc.) denote the set of $\gamma \in \Gamma$ satisfying $\gamma<\beta$ (resp., $\gamma \leq \beta, \gamma>\beta$, etc.).

(2) For a subset $M \subseteq N, M_{<\beta}$ (resp., $M_{\leq \beta}, M_{>\beta}$, etc.) denotes the set of $m \in M$ satisfying $\varphi(m)<\beta$ (resp., $\varphi(m) \leq \beta, \varphi(m)>\beta$, etc.).

(3) For a subfield $L$ of $D$, write $N_{L}:=N \cap L, \varphi_{L}:=\left.\varphi\right|_{N_{L}}: N_{L} \rightarrow \Gamma_{L}$, where $\Gamma_{L}:=\varphi\left(N_{L}\right)$.

\footnotetext{
${ }^{2}$ The following conventions will be kept throughout the paper: the operation on $\Gamma$ will be denoted additively, though $\Gamma$ is not assumed to be commutative; the order relation will be denoted $\leq$ (so, for any $\alpha, \beta, \gamma, \delta \in \Gamma$ with $\alpha \leq \gamma$ and $\beta \leq \delta$ we have $\alpha+\beta \leq \gamma+\delta$ ).
} 
(4) Given a (surjective) valuation $v: D^{\times} \rightarrow \tilde{\Gamma}$ (note that $\tilde{\Gamma}$ is necessarily abelian; cf. Remark 2.2 in [20]), we let $\mathcal{O}_{D, v}=\left\{x \in D^{\times} \mid v(x) \geq 0\right\} \cup\{0\}$ denote the valuation ring of $v$. For any $\delta \in(\tilde{\Gamma})_{>0}, \mathfrak{m}_{D, v}(\delta)=\left\{x \in D^{\times} \mid v(x)>\delta\right\} \cup\{0\}$ will denote the corresponding ideal of $\mathcal{O}_{D, v}$. These ideals form a fundamental system of open neighborhoods of zero for the natural topology on $D$ associated with $v$ which is sometimes referred to as the $v$-adic topology. We will write $\mathcal{O}_{v}$, or simply $\mathcal{O}$, instead of $\mathcal{O}_{D, v}$ if this will not lead to confusion.

Theorem 5.1 of [20] asserts that given a nontrivial valuation-like map $\varphi: N \rightarrow \Gamma$ to a totally ordered group $\Gamma$, then (if $K$ is a finitely generated field) there exists a height one valuation $v$ of $D$ associated with $\varphi$, and $N$ is open in the $v$-adic topology. In $\S \S 2$ - 3 we extend this result and prove:

Theorem 2.3. Suppose $K$ is finitely generated and that $\varphi: N \rightarrow \Gamma$ is a surjective homomorphism onto a nontrivial partially ordered group $\Gamma$. Let $\mathcal{R}$ be the subring of $D$ generated by $N_{\geq 0}$. Assume that

(1) $N_{>0} \not \subseteq K$;

(2) $\Gamma_{K}:=\varphi\left(N \cap K^{\times}\right)$is a nontrivial totally ordered group;

(3) $\varphi$ is a leveled map having a level $\alpha \in\left(\Gamma_{K}\right)_{\geq 0}$;

(4) $\mathcal{R}$ is a proper subring of $D$.

Then there exists a height one valuation $v: D^{\times} \rightarrow \tilde{\Gamma}$ associated with $\varphi$. Suppose in addition that

$\left(4^{\prime}\right)$ there exists $\gamma \in \Gamma_{\geq 0}$ such that $\mathcal{R} \cap N \subseteq N_{>-\gamma}$

(which in particular implies (4)). Then $N$ is open with respect to the corresponding $v$-adic topology.

In this section we show that under hypotheses (1) - (4) of Theorem 2.3, there exists a height one valuation $v$ associated with $\varphi$. The next section focuses on proving that if in addition we assume hypothesis $\left(4^{\prime}\right)$, then $N$ is open with respect to any valuation associated with $\varphi$ (Theorem 3.1).

The assumptions that the group $\Gamma_{K}$ is totally ordered and the homomorphism $\varphi_{K}: N_{K} \rightarrow \Gamma_{K}$ admits a level $\alpha \in \Gamma_{K}$ mean that $\varphi_{K}$ is a valuation-like map in the sense of 20]. By Theorem 4.1.1 and Proposition 2.6 in [20], there exists a nontrivial valuation $v_{0}: K^{\times} \rightarrow \tilde{\Gamma}_{0}$ associated with $\varphi_{K}$, and $N_{K}$ is open in $K^{\times}$with respect to the topology defined by this valuation, i.e. there exists $\delta \in\left(\tilde{\Gamma}_{0}\right)_{\geq 0}$ such that

$$
1+\mathfrak{m}_{K, v_{0}}(\delta) \subseteq N_{K},
$$

where $\mathfrak{m}_{K, v_{0}}(\delta)=\left\{x \in K^{\times} \mid v_{0}(x)>\delta\right\} \cup\{0\}$. Furthermore, by Theorem 4.1.2 in [20], since $K$ is finitely generated, we may (and we will) assume that the height of $v_{0}$ is one. We will show that $v_{0}$ (uniquely) extends to a valuation $v: D^{\times} \rightarrow \tilde{\Gamma}$, and that this valuation is associated with $\varphi$.

We pick a basis $a_{1}, \ldots, a_{n^{2}}$ of $D$ over $K\left(\right.$ where $n^{2}=\operatorname{dim}_{K} D$ ) and we define a norm \|\|$_{v_{0}}$ on $D$ by

$$
\left\|\alpha_{1} a_{1}+\cdots+\alpha_{n^{2}} a_{n^{2}}\right\|_{v_{0}}=\max _{i=1, n^{2}}\left|\alpha_{i}\right|_{v_{0}},
$$

where ||$_{v_{0}}$ is the absolute value associated with $v_{0}$. (One easily shows that two norms of the form (iv) constructed using different bases are equivalent, hence the corresponding notions of boundedness and openness coincide.) The existence of an extension of $v_{0}$ will be derived from the following result analogous to Theorem 5.2 in [20]. 
Theorem 2.4. Let $D$ be a central division algebra of degree $n$ over an arbitrary field $K$, and let $v_{0}$ be a valuation of $K$ having height one. Assume there exists a subring $\mathcal{B} \varsubsetneqq D$ such that

(a) $\mathcal{B}$ is open in $D$ with respect to the topology defined by the norm \|\|$_{v_{0}}$;

(b) there exists a positive integer $k$ such that $d \mathcal{B} d^{-1} \subseteq \mathcal{B}$ for all $d \in\left(D^{\times}\right)^{k}$ $=\left\{x^{k} \mid x \in D^{\times}\right\}$.

Then $v_{0}$ extends to a height one valuation $v$ of $D$ such that $\mathcal{B}$ is contained in the corresponding valuation ring $\mathcal{O}_{v}$.

Proof. Let $A=D \otimes_{K} K_{v_{0}}$, where $K_{v_{0}}$ is the completion of $K$ with respect to $v_{0}$. Then $A \simeq M_{d}(\mathcal{D})$ for some integer $d \geq 1$ and some central division algebra $\mathcal{D}$ over $K_{v_{0}}$. The valuation $v_{0}$ extends from $K_{v_{0}}$ to a valuation $u$ on $\mathcal{D}$ by the formula

$$
u(x)=\frac{1}{l} v_{0}\left(\operatorname{Nrd}_{\mathcal{D} / K_{v_{0}}}(x)\right) \text { for any } \quad x \in \mathcal{D}^{\times},
$$

where $l$ is the degree of $\mathcal{D}$ (cf., for example, 14]), so it suffices to establish that our assumptions force $d=1$, since then $\mathcal{D}=D \otimes_{K} K_{v_{0}}$ and the restriction $\left.u\right|_{D}$ provides a height one extension of $v_{0}$. Note that since the height of $v_{0}$ is one, the definition of $u$ makes sense and the height of $u$ is one; in particular, $u$ admits an associated absolute value ||$_{u}$.

The norm \|\|$_{v_{0}}$ extends from $D$ to $A$ by means of equation (iv) (just think of $\alpha_{1}, \ldots, \alpha_{n^{2}}$ as elements of $K_{v_{0}}$ ), and we saw in the proof of Theorem 5.2 in [20] that the closure $\overline{\mathcal{B}}$ of $\mathcal{B}$ in $A$ is a proper open subring. Then according to Lemma 5.3 in [20], $\overline{\mathcal{B}}$ is bounded. To obtain a contradiction if $d>1$, we will use a different norm on $A \simeq M_{d}(\mathcal{D})$ :

$$
\left\|\left(a_{i j}\right)\right\|_{u}=\max _{i, j=1, d}\left|a_{i j}\right|_{u} .
$$

Since both \|\|$_{v_{0}}$ and \|\|$_{u}$ are norms on $A$ as a vector space over $K_{v_{0}}$, they are equivalent because $\operatorname{dim}_{K_{v_{0}}} A<\infty$ and $K_{v_{0}}$ is complete (cf. [10]); in particular, they give rise to the same notion of boundedness on $A$.

It follows from assumption (b) in the statement of the theorem that $a \overline{\mathcal{B}} a^{-1} \subseteq \overline{\mathcal{B}}$ for any $a \in\left(A^{\times}\right)^{k}$. Now, suppose $d>1$. Since the subring $\overline{\mathcal{B}} \subseteq M_{d}(\mathcal{D})$ is open, it contains $M_{d}\left(\mathfrak{m}_{\mathcal{D}, u}(\delta)\right)$, for some nonnegative $\delta$ in the value group of $u$, in particular there exists $b=\left(b_{i j}\right) \in \overline{\mathcal{B}}$ with $b_{12} \neq 0\left(\right.$ as $\left.\mathfrak{m}_{\mathcal{D}, u}(\delta) \neq 0\right)$. Also pick $s \in \mathcal{D}^{\times}$so that $|s|_{u}>1$, and let $t=\operatorname{diag}\left(s, s^{-1}, 1, \ldots, 1\right) \in A^{\times}$. Now consider the sequence

$$
d_{l}:=t^{l k} \in\left(A^{\times}\right)^{k}, \quad l=1,2, \ldots .
$$

Then for $b_{l}=d_{l} b d_{l}^{-1} \in \overline{\mathcal{B}}$, the (12)-entry is $\left(b_{l}\right)_{12}=s^{l k} b_{12} s^{l k}$, so $\left|\left(b_{l}\right)_{12}\right|_{u} \rightarrow \infty$ as $l \rightarrow \infty$, contradicting the boundedness of $\overline{\mathcal{B}}$. Thus, $d=1$, i.e. $A=\mathcal{D}$, and the restriction $v=\left.u\right|_{D}$ provides a height one extension of $v_{0}$. Since $\overline{\mathcal{B}}$ is bounded, it is contained in the valuation ring of $u$ (because for an element $x \in \mathcal{D}^{\times}$which is not in the valuation ring of $u$ one has $\left|x^{l}\right|_{u} \rightarrow \infty$ as $\left.l \rightarrow \infty\right)$, implying the inclusion $\mathcal{B} \subseteq \mathcal{O}_{v}$.

To prove the existence of $v$ asserted in Theorem 2.3. we will apply Theorem 2.4 to the subring $\mathcal{R} \subseteq D$ generated by $N_{\geq 0}$. The following proposition establishes the properties required for its application. 
Proposition 2.5. Let $\varphi: N \rightarrow \Gamma$ be a (nontrivial surjective) leveled map having a level $\alpha \in\left(\Gamma_{K}\right)_{\geq 0}$. Assume that the group $\Gamma_{K}$ is nontrivial and totally ordered, so there exists a valuation $v_{0}: K^{\times} \rightarrow \tilde{\Gamma}_{0}$ associated with $\varphi_{K}$. Then

(1) there exists $\varepsilon \in\left(\tilde{\Gamma}_{0}\right)_{\geq 0}$, such that the subring $\mathcal{R}_{K} \subseteq K$ generated by $\left(N_{K}\right)_{\geq 0}$ contains $\mathfrak{m}_{K, v_{0}}(\varepsilon)$.

Suppose in addition that $N_{\geq 0} \nsubseteq K$. Then

(2) $N_{\geq 0}$ contains a basis of $D$ over $K$;

(3) if $v_{0}$ has height one, then the subring $\mathcal{R} \subseteq D$ generated by $N_{\geq 0}$ is open with respect to the topology defined by the norm \|\|$_{v_{0}}$ (see (iv)).

Proof. (1) Let $\delta \in\left(\tilde{\Gamma}_{0}\right)_{\geq 0}$ be as in (iii). We pick $c \in N_{K}$ such that $v_{0}(c)>0$ (which exists since $N_{K}$ has finite index in $K^{\times}$). Then $c\left(1+\mathfrak{m}_{K, v_{0}}(\delta)\right) \subseteq N_{K}$ and $v_{0}\left(c\left(1+\mathfrak{m}_{K, v_{0}}(\delta)\right)\right) \subseteq\left(\tilde{\Gamma}_{0}\right)_{>0}$. Since $\Gamma_{K}$ is totally ordered and $v_{0}$ is associated with $\varphi_{K}$, this implies that

$$
c\left(1+\mathfrak{m}_{K, v_{0}}(\delta)\right) \subseteq\left(N_{K}\right)_{>0} \subseteq \mathcal{R}_{K},
$$

i.e. $c \mathfrak{m}_{K, v_{0}}(\delta) \subseteq \mathcal{R}_{K}$. Then $\varepsilon:=\delta+v_{0}(c)$ is as required.

(2) Let $V$ be the $K$-linear span of $N_{\geq 0}$. Since $N_{\geq 0}$ is closed under multiplication, $V$ is a subring of $D$; moreover, $V$ is invariant under conjugation by $N$. Let $\Omega$ be an algebraically closed field containing $K$. Since $N$ has finite index in $D^{\times}$, it is Zariski dense in $\left(D \otimes_{K} \Omega\right)^{\times} \simeq G L_{n}(\Omega)$. Thus, $V \otimes_{K} \Omega$ is a subalgebra of $D \otimes_{K} \Omega \simeq M_{n}(\Omega)$ invariant under conjugation by $G L_{n}(\Omega)$. However, there are no proper noncentral conjugation invariant subalgebras $R \subseteq M_{n}(\Omega)$. (For the sake of completeness, we recall a proof of this well-known fact. Let $T$ be the diagonal torus in $G L_{n}(\Omega)$. Then $R$ is not centralized by $T$ as otherwise it would be centralized by all semisimple elements in $G L_{n}(\Omega)$, and therefore by $G L_{n}(\Omega)$ itself. Thus, $R$ contains an eigenvector for $T$ for some nontrivial character, i.e. an off-diagonal element $e_{i j}$, $i \neq j$, of the standard basis of $M_{n}(\Omega)$. But all off-diagonal $e_{i j}$ 's are conjugate under $G L_{n}(\Omega)$, so $R$ contains all of them. Finally, $e_{i j} e_{j i}=e_{i i}$, which puts the diagonal elements of the standard basis inside $R$ as well.) Since $N_{\geq 0} \nsubseteq K$, we conclude that $V \otimes_{K} \Omega=M_{n}(\Omega)$, and therefore $V=D$, as required.

(3) According to (2), $N_{\geq 0}$ contains a basis $\omega_{1}, \ldots, \omega_{n^{2}}$ of $D$ over $K$. Let $\varepsilon$ be as in (1). Then

$$
\mathfrak{m}_{K, v_{0}}(\varepsilon) \omega_{1}+\cdots+\mathfrak{m}_{K, v_{0}}(\varepsilon) \omega_{n^{2}} \subseteq \mathcal{R},
$$

giving the openness of $\mathcal{R}$ in $D$.

Proof of the existence of the valuation $v$ asserted in Theorem 2.3. According to condition (4) in the statement of Theorem 2.3, the subring $\mathcal{B}=\mathcal{R}$ is proper. By Proposition 2.5(3), it is also open with respect to the topology defined by the norm \|\|$_{v_{0}}$, i.e. satisfies condition (a) in Theorem 2.4. Furthermore, since $\Gamma$ is an ordered group, $\Gamma_{\geq 0}$ is a normal subset of $\Gamma$, implying that $N_{\geq 0}$ is a normal subset of $N$, so $\mathcal{R}$ is normalized by $N$. Since $N \supseteq\left(D^{\times}\right)^{k}$ for $k=\left(\left|D^{\times}: N\right|\right)$ !, condition (b) holds as well. Thus, according to Theorem 2.4, $v_{0}$ extends to a height one valuation $v: D^{\times} \rightarrow \tilde{\Gamma}$ and, moreover, $\mathcal{R}$ is contained in its valuation ring $\mathcal{O}_{v}$. The latter means that $N_{\geq 0} \subseteq \mathcal{O}_{v}$, i.e. for $x \in N, \varphi(x) \geq 0$ implies $v(x) \geq 0$. We conclude that $v(\operatorname{Ker} \varphi)=\{0\}$, and the arising homomorphism $\theta: \Gamma \rightarrow \tilde{\Gamma}$ is, in fact, a homomorphism of ordered groups, so $v$ is associated with $\varphi$. 


\section{THE OPENNESS THEOREM}

To conclude the proof of Theorem [2.3, it remains to establish the openness of $N$. In [20, Prop. 2.6, we showed that if $N$ admits a valuation-like map $\varphi: N \rightarrow \Gamma$ with $\Gamma$ totally ordered, then $N$ is open in $D^{\times}$with respect to any valuation $v$ of $D$ associated with $\varphi$. In this section we will prove a similar result which, in particular, completes the proof of Theorem 2.3 .

Theorem 3.1. Let $\varphi: N \rightarrow \Gamma$ be a surjective homomorphism onto a partially ordered group satisfying conditions $(1)-(3)$ and $\left(4^{\prime}\right)$ in the statement of Theorem 2.3. If a valuation $v: D^{\times} \rightarrow \tilde{\Gamma}$ is associated with $\varphi$, then $N$ is open in $D^{\times}$with respect to the $v$-adic topology.

(We note that the assumption, made in Theorem[2.3, that $K$ be finitely generated is not necessary for the validity of Theorem 3.1.) One of the ingredients of the proof is the following elaboration on Proposition 2.6 in [20].

Proposition 3.2. Let $\varphi: N \rightarrow \Gamma$ be a leveled map admitting an associated valuation $v: D^{\times} \rightarrow \tilde{\Gamma}$. Assume that for the valuation ring $\mathcal{O}_{v}$ of $v$ there exists $\beta \in \Gamma_{\geq 0}$ such that

$$
\mathcal{O}_{v} \cap N \subseteq N_{>-\beta}
$$

Then $N$ is open in $D^{\times}$with respect to the $v$-adic topology.

Proof. Let $\alpha \in \Gamma_{\geq 0}$ be a level of $\varphi$. We need to show that there exists a $\delta \in \tilde{\Gamma}_{\geq 0}$ such that

$$
1+\mathfrak{m}(\delta) \subseteq N
$$

where $\mathfrak{m}(\delta)=\left\{x \in D^{\times} \mid v(x)>\delta\right\} \cup\{0\}$. For that we will show that for each coset $N a$ of $N$ in $D^{\times}$, there exists $\gamma(N a)=\gamma \in \tilde{\Gamma}_{\geq 0}$ such that

$$
1+(N a \cap \mathfrak{m}(\gamma)) \subseteq N
$$

Then, since $N$ has a finite index in $D^{\times}$, the maximum $\delta=\max \gamma(N a)$, taken over all cosets of $N$ in $D^{\times}$, exists (recall that $\tilde{\Gamma}$ is totally ordered!) and obviously satisfies (ii). Since $v$ is associated with $\varphi$, there exists a nontrivial homomorphism of ordered groups $\theta: \Gamma \rightarrow \tilde{\Gamma}$ for which the diagram (ii) of $\S 2$ is commutative. To establish the existence of $\gamma(N a)$, we need the following.

Lemma 3.3. (1) For $m, n \in N$ such that $v(m)<v(n)-\theta(\alpha+\beta)$, the element $c=m+n$ belongs to $N$.

(2) For any $d \in D^{\times}$, there exists $\beta_{d} \in \tilde{\Gamma}$ such that

$$
d+\left\{n \in N \mid v(n)<\beta_{d}\right\} \subseteq N
$$

Proof. (1) Let $\alpha \in \Gamma_{\geq 0}$ be a level of $\varphi$, and let $\beta \in \Gamma_{\geq 0}$ be as in Proposition 3.2. Pick $a, b \in N$ so that $\varphi(a)=\alpha$ and $\varphi(b)=\beta$; then $v(a)=\theta(\alpha)$ and $v(b)=\theta(\beta)$. We have $v\left(m^{-1} n a^{-1} b^{-1}\right)>0$ (note that $\tilde{\Gamma}$ is commutative!), so $m^{-1} n a^{-1} b^{-1} \in \mathcal{O}_{v}$, hence $\varphi\left(m^{-1} n a^{-1} b^{-1}\right)>-\beta=\varphi\left(b^{-1}\right)$ according to (i). It follows that $\varphi\left(m^{-1} n a^{-1}\right)>0$, and therefore $\varphi\left(n^{-1} m\right)<\varphi\left(a^{-1}\right)=-\alpha$, i.e. $n^{-1} m \in N_{<-\alpha}$. Now,

$$
n^{-1} m+1 \in N_{<-\alpha}+1 \subseteq N_{<-\alpha} \subseteq N \text {, }
$$

yielding $c=n\left(n^{-1} m+1\right) \in N$. 
(2) Since $D$ is infinite, $D=N-N$ (cf. 6], 33]), so there exists $s \in N$ such that $d+s \in N$. Set

$$
\beta_{d}=\min (v(s), v(d+s))-\theta(\alpha+\beta) .
$$

Suppose now that $t \in N$ satisfies $v(t)<\beta_{d}$. Then, in particular, $v(t)<v(s)-$ $\theta(\alpha+\beta)$, so it follows from (1) that $t-s \in N$ (observe that $v(-s)=v(s)$ ). Moreover, since $\alpha, \beta \in \Gamma_{\geq 0}$, we have $v(t)<v(s)$, and therefore $v(t-s)=v(t)$ as $v$ is a valuation. Thus, $v(t-s)<v(d+s)-\theta(\alpha+\beta)$, and

$$
d+t=(d+s)+(t-s) \in N
$$

again according to (1). The proof of the lemma is complete.

Now, fix a representative $a$ of a given coset $N a$ and let

$$
\gamma=\gamma(N a):=|v(a)|+\left|\beta_{a}\right|,
$$

where $\beta_{a}$ is as in Lemma [3.3) (2) (here, as usual, for $\gamma \in \tilde{\Gamma}$, we denote $|\gamma|=$ $\max \{\gamma,-\gamma\})$. Suppose $n a \in N a \cap \mathfrak{m}(\gamma)$. Then

$$
v(n)=v(n a)-v(a)>\left(|v(a)|+\left|\beta_{a}\right|\right)-v(a) \geq\left|\beta_{a}\right|,
$$

implying that

$$
1+n a=n\left(n^{-1}+a\right) \in N
$$

as $v\left(n^{-1}\right)<-\left|\beta_{a}\right| \leq \beta_{a}$, and therefore by Lemma $3.3(2), n^{-1}+a \in N$. This proves (iii) and completes the proof of Proposition [3.2.

Remark 3.4. Proposition 3.2 immediately generalizes to the situation when there is a finite set $T=\left\{v_{1}, \ldots, v_{r}\right\}$ of valuations associated with a given leveled map $\varphi: N \rightarrow \Gamma:$ if $\mathcal{O}_{v_{i}}$ is the valuation ring of $v_{i}$ and $\mathcal{O}_{T}=\bigcap_{i=1}^{r} \mathcal{O}_{v_{i}}$, then the condition

$$
N \cap \mathcal{O}_{T} \subseteq N_{>-\beta},
$$

for some $\beta \in \Gamma_{\geq 0}$, implies that $N$ is open in $D^{\times}$with respect to the topology defined by $T$.

Proof of Theorem 3.1. By the definition of an associated valuation, $v$ is nontrivial. In view of Proposition 3.2 , all we have to show is that under the assumptions made in the statement of the theorem there exists $\beta \in \Gamma_{\geq 0}$, satisfying (i). We will need the following.

Lemma 3.5. Let $\mathcal{O} \subseteq K$ be an integrally closed ring, and let $\tilde{\mathcal{O}} \subseteq D$ be a subring which is integral over $\mathcal{O}$ and contains a basis $\omega_{1}, \ldots, \omega_{n^{2}}$ of $D / K$. Then there exists $t \in \mathcal{O}, t \neq 0$, such that

$$
\tilde{\mathcal{O}} \subseteq \frac{1}{t}\left(\mathcal{O} \omega_{1}+\cdots+\mathcal{O} \omega_{n^{2}}\right)
$$

Proof. The equation $h(x, y):=\operatorname{Trd}_{D / K}(x y)$ defines a nondegenerate symmetric bilinear form on $D$ as a vector space over $K$. Since $\mathcal{O}$ is integrally closed, $\operatorname{Trd}_{D / K}(\tilde{\mathcal{O}})$ $\subseteq \mathcal{O}$ (indeed, suppose $a \in \tilde{\mathcal{O}}$, and let $L \subseteq D$ be a maximal subfield containing $a$; then $a$ belongs to $\mathcal{O}_{L}:=$ integral closure of $\mathcal{O}$ in $L$, so the required inclusion follows from the following two facts: 1) $\operatorname{Trd}_{D / K}(a)=\operatorname{Tr}_{L / K}(a)$ (cf. [5]); 2) $\operatorname{Tr}_{L / K}\left(\mathcal{O}_{L}\right) \subseteq \mathcal{O}$ (cf. 5], Ch. V, $\S 1, \mathrm{n}^{\circ} 6$, cor. 2)). Let $t=\operatorname{det}\left(h\left(\omega_{i}, \omega_{j}\right)\right)$; then $t \in \mathcal{O}, t \neq 0$. Take an arbitrary $a \in \tilde{\mathcal{O}}$ and write it as

$$
a=\alpha_{1} \omega_{1}+\cdots+\alpha_{n^{2}} \omega_{n^{2}}
$$


with $\alpha_{i} \in K$. We need to show that in fact $\alpha_{i} \in \frac{1}{t} \mathcal{O}$ for all $i$. However, the $\alpha_{i}$ 's can be determined from the linear system

$$
\sum_{i=1}^{n^{2}} \alpha_{i} h\left(\omega_{i}, \omega_{j}\right)=A_{j}, \quad j=1, \ldots, n^{2},
$$

where $A_{j}:=\operatorname{Trd}_{D / K}\left(a \omega_{j}\right) \in \mathcal{O}$. Since the determinant of this system is $t$, we obtain from Cramer's Rule that $\alpha_{i} \in \frac{1}{t} \mathcal{O}$, as required.

Let $\mathcal{O}_{v_{0}}=\mathcal{O}_{v} \cap K$ be the valuation ring of the restriction $v_{0}=\left.v\right|_{K}: K^{\times} \rightarrow$ $v\left(K^{\times}\right)=: \tilde{\Gamma}_{0}$ (we note that $v_{0}$ is nontrivial as $v$ is such and $[D: K]<\infty$, and that $v_{0}$ is associated with $\varphi_{K}$ ). To apply Lemma 3.5, we observe that $\mathcal{O}_{v}$ is integral over $\mathcal{O}_{v_{0}}$. Indeed, let $a \in \mathcal{O}_{v}$ and let $L$ be a maximal subfield of $D$ containing $a$. The existence of a valuation of $D$ extending $v_{0}$ implies that $\left.v\right|_{L}$ is the only extension of $v_{0}$ to $L$ (cf. [34]), with $\mathcal{O}_{v} \cap L$ as the valuation ring. On the other hand, it is known (cf. [5], Ch. VI, $\S 1, \mathrm{n}^{\circ} 3$, cor. 3) that the integral closure of $\mathcal{O}_{v_{0}}$ in $L$ coincides with the intersection of the valuation rings of all extensions of $v_{0}$ to $L$. Thus, $\mathcal{O}_{v} \cap L$ is integral over $\mathcal{O}_{v_{0}}$, and eventually $\mathcal{O}_{v}$ is integral over $\mathcal{O}_{v_{0}}$. We also note that $\mathcal{O}_{v_{0}}$, being a valuation ring, is integrally closed (cf. [5], Ch. VI, $\S 1, \mathrm{n}^{\circ} 3$, cor. 1). Since $N_{\geq 0} \nsubseteq K$, according to Proposition [2.5)(2) there exists a basis $\omega_{1}, \ldots, \omega_{n^{2}} \in N_{\geq 0} \subseteq \mathcal{O}_{v}$. Applying Lemma 3.5 we obtain that there exists a nonzero $t \in \mathcal{O}_{v_{0}}$ such that

$$
\mathcal{O}_{v} \subseteq \frac{1}{t}\left(\mathcal{O}_{v_{0}} \omega_{1}+\cdots+\mathcal{O}_{v_{0}} \omega_{n^{2}}\right) .
$$

Using $\frac{1}{t}=\frac{t^{d-1}}{t^{d}}$, we can replace $t$ with $t^{d}$ in (iv), where $d=\left|K^{\times}: N_{K}\right|$, and assume that $t \in N_{K}$. In fact, we may (and we will) even assume that $t \in\left(N_{K}\right)_{\geq 0}$. Indeed, if $\varphi(t)<0$, then because $t \in \mathcal{O}_{v_{0}}$ and $v_{0}$ is associated with $\varphi$, one has $v(t)=0$, so one can simply take $t=1$ in (iv). Furthermore, let $\varepsilon \in\left(\tilde{\Gamma}_{0}\right)_{\geq 0}$ be as in Proposition 2.5 (1), i.e. $\mathfrak{m}_{K, v_{0}}(\varepsilon) \subseteq \mathcal{R}_{K}$ (the subring generated by $\left.\left(N_{K}\right)_{\geq 0}\right)$. Since $\left|\tilde{\Gamma}_{0}: v_{0}\left(N_{K}\right)\right|<\infty$, one can pick $s \in\left(N_{K}\right)_{>0}$ satisfying $v(s)>\varepsilon$. Then $s \mathcal{O}_{v_{0}} \subseteq \mathcal{R}_{K}$. Now, set $f=s t$. Then $f \in\left(N_{K}\right)_{>0}$ and

$$
\mathcal{O}_{v} \subseteq \frac{1}{f}\left(\mathcal{R}_{K} \omega_{1}+\cdots+\mathcal{R}_{K} \omega_{n^{2}}\right) \subseteq \frac{1}{f} \mathcal{R}
$$

where $\mathcal{R} \subseteq D$ is the subring generated by $N_{\geq 0}$. It follows now from condition ( $\left.4^{\prime}\right)$ in the statement of Theorem 2.3 that

$$
\mathcal{O}_{v} \cap N \subseteq \frac{1}{f}(\mathcal{R} \cap N) \subseteq \frac{1}{f} N_{>-\gamma} \subseteq N_{>-\beta}
$$

for $\beta=\gamma+\varphi(f) \in \Gamma_{\geq 0}$, verifying the required assumption in Proposition 3.2 and completing the proof of Theorem 3.1.

\section{Strongly LeVELEd MAPS}

The purpose of this section and of the following $\$ 5$ is to show that when $N \triangleleft$ $D^{\times}$and $D^{\times} / N$ satisfies Property $\left(3 \frac{1}{2}\right), N$ admits a leveled map satisfying all the hypotheses $(1)-(3)$ and $\left(4^{\prime}\right)$ made in Theorem [2.3. In fact we will show in $\S 5$ that the mere assumption that $\Delta_{D^{\times} / N}$ has diameter $\geq 3$ implies the existence of a strongly leveled map $\varphi: N \rightarrow \Gamma$, which we now define. 
Definition 4.1. Let $\varphi: N \rightarrow \Gamma$ be a surjective homomorphism onto a partially ordered group $\Gamma$ (with $\Gamma_{>0} \neq \emptyset$ ). We say that $\varphi$ is a strongly leveled map (or an $s$-leveled map for short) if there exists $\alpha \in \Gamma_{\geq 0}$ (called an s-level of $\varphi$ ) such that

$$
1 \pm N_{>\alpha} \subseteq N_{\leq 0}
$$

We note that although we keep the assumption $-1 \in N$, we are not assuming that $-1 \in \operatorname{Ker} \varphi$, which explains the presence of \pm in (SL). Now, if $\alpha \in \Gamma_{\geq 0}$ is an s-level of $\varphi$, then for any $n \in N_{<-\alpha}$ one has

$$
\varphi(1+n)=\varphi\left(n\left(1+n^{-1}\right)\right) \leq \varphi(n)<-\alpha
$$

as $1+n^{-1} \in 1+N_{>\alpha} \subseteq N_{\leq 0}$, i.e. $1+n \in N_{<-\alpha}$. Thus, any s-leveled map is leveled (with the same level). We also observe that given an s-level, say $\alpha$, of $\varphi$, any $\beta \in \Gamma_{\geq \alpha}$ is an s-level of $\varphi$ as well.

Let $\varphi: N \rightarrow \Gamma$ be an s-leveled map having an s-level $\alpha$. We let $\mathcal{A}$ (resp. $\mathcal{R}$ ) denote the subring of $D$ generated by $N_{>\alpha}$ (resp. by $N_{\geq 0}$ ); obviously, $\mathcal{A}$ (resp. $\mathcal{R}$ ) coincides with the set of all elements of the form $\epsilon_{1} a_{1}+\cdots+\epsilon_{l} a_{l}$ with $\epsilon_{i}= \pm 1$ and $a_{i} \in N_{>\alpha}$ (resp. $a_{i} \in N_{\geq 0}$ ). We also set $U=\operatorname{Ker} \varphi$.

The arguments in $\S 5$ and the hypotheses of Theorem 2.3 involve certain properties of the rings $\mathcal{A}$ and $\mathcal{R}$ and of s-leveled maps. Some of these properties will be established in this section.

Proposition 4.2. (1) $1 \pm N_{>\alpha} \subseteq U$;

(2) $U \pm \mathcal{A} \subseteq U$;

(3) $1 \notin \mathcal{A}$;

(4) for any $m \in \mathcal{A} \cap N_{\geq 0}$, the element $\beta:=\varphi(m)$ is an s-level for $\varphi$.

Proof. (1) Let $n \in N_{>\alpha}$ and $\epsilon \in\{1,-1\}$. Then $1+\epsilon n \in N_{\leq 0}$ by the definition of an s-leveled map, so we need to show only that $(1+\epsilon n)^{-1} \in N_{\leq 0}$. We notice that

$$
(1+\epsilon n)^{-1}=1-\epsilon n(1+\epsilon n)^{-1} .
$$

Since $1+\epsilon n \in N_{\leq 0}$, we obtain that $n(1+\epsilon n)^{-1} \in N_{>\alpha}$, so it follows from (ii) that $(1+\epsilon n)^{-1} \in N_{\leq 0}$, as required.

(2)\&(3) Since $U N_{>\alpha} \subseteq N_{>\alpha}$, we obtain using (1) that for $u \in U$ and $n \in N_{>\alpha}$, one has

$$
u \pm n=u\left(1 \pm u^{-1} n\right) \in U .
$$

Thus, $U \pm N_{>\alpha} \subseteq U$, and (2) follows. As $0=1-1 \notin U$, (2) implies (3).

(4) If $n \in N_{>\beta}$, where $\beta=\varphi(m)$, then $n m^{-1} \in N_{\geq 0}$, implying that $n=$ $\left(n m^{-1}\right) m \in \mathcal{A}$. Thus, $N_{>\beta} \subseteq \mathcal{A}$, and then by (2), $1 \pm N_{>\beta} \subseteq N_{\leq 0}$, hence, by definition, $\beta$ is an s-level for $\varphi$.

Proposition 4.3. Let $\varphi: N \rightarrow \Gamma$ be a strongly leveled map having an s-level $\alpha$. Then

(1) $\mathcal{R} \cap N_{<-\alpha}=\emptyset$; in particular $\mathcal{R} \neq D$.

(2) If $K^{\times} \subseteq N$ and the subgroup $\Gamma_{K}:=\varphi\left(K^{\times}\right)$is totally ordered, then $\Gamma_{K} \neq\{0\}$ and $\varphi$ possesses an s-level belonging to $\left(\Gamma_{K}\right)_{\geq 0}$.

(3) If there exists $\mu \in \Gamma_{\geq 0}$ such that $\mathcal{A} \cap N \subseteq N_{>-\mu}$, then there exists $\gamma \in \Gamma_{\geq 0}$ such that $\mathcal{R} \cap N \subseteq N_{>-\gamma}$. 
Proof. (1) The inclusion $N_{>0} N_{>\alpha} \subseteq N_{>\alpha}$ implies that $\mathcal{R} \mathcal{A} \subseteq \mathcal{A}$. Now, if $z \in$ $\mathcal{R} \cap N_{<-\alpha}$, then $z^{-1} \in N_{>\alpha} \subseteq \mathcal{A}$, so $1=z z^{-1} \in \mathcal{A}$, contradicting Proposition $4.2(3)$.

(2) We take an arbitrary $s \in N_{>\alpha}$, and let $p(x)=a_{t} x^{t}+a_{t-1} x^{t-1}+\cdots+a_{0}$ be the minimal polynomial of $s$ over $K$. Since $\Gamma_{K}=\varphi\left(K^{\times}\right)$is totally ordered, $\delta=\min \left\{\varphi\left(a_{i}\right) \mid a_{i} \neq 0\right\}$ is defined; we choose an index $j$ so that $\delta=\varphi\left(a_{j}\right)$. Multiplying by $a_{j}^{-1}$, we obtain the relation

$$
b_{t} s^{t}+\cdots+b_{0}=0
$$

where $b_{i}=a_{j}^{-1} a_{i}$ and $b_{i} \in N_{\geq 0}$ for all $i$ such that $b_{i} \neq 0$. Of course $b_{0} \neq 0$, and we have

$$
b_{0}=-\left(b_{t} s^{t}+\cdots+b_{1} s\right) \in \mathcal{A} \cap N_{\geq 0} .
$$

Then according to Proposition 4.2 $(4), \beta=\varphi\left(b_{0}\right) \in\left(\Gamma_{K}\right)_{\geq 0}$ is an s-level for $\varphi$. Furthermore, $b_{0} \notin U$, as otherwise we would have $1=b_{0}^{-1} b_{0} \in U \mathcal{A} \subseteq \mathcal{A}$, contradicting Proposition 4.2(3). Thus, $\Gamma_{K} \simeq K^{\times} /\left(K^{\times} \cap U\right)$ is nontrivial.

(3) Again, take an arbitrary $s \in N_{>\alpha}$. Then $s N_{\geq 0} \subseteq N_{>\alpha}$, implying $s \mathcal{R} \subseteq \mathcal{A}$. Thus, $\mathcal{R} \cap N \subseteq\left(s^{-1} \mathcal{A}\right) \cap N=s^{-1}(\mathcal{A} \cap N)$. It follows that

$$
\mathcal{R} \cap N \subseteq s^{-1}(\mathcal{A} \cap N) \subseteq s^{-1} N_{>-\mu} \subseteq N_{>-\gamma}
$$

for $\gamma:=\mu+\varphi(s)$.

\section{Strongly Leveled maps in diameter $\geq 3$ And Property $\left(3 \frac{1}{2}\right)$}

In this section $-1 \in N \subseteq D^{\times}$is a normal subgroup of finite index. The purpose of this section is to show that the mere hypothesis that $\operatorname{diam}\left(\Delta_{D^{\times} / N}\right) \geq 3$ implies the existence of a strongly leveled map $\varphi: N \rightarrow \Gamma$ having an s-level $\alpha$. Furthermore, $\mathcal{A} \cap N \subseteq N_{>-\mu}$, for some $\mu \in \Gamma_{\geq 0}$, where $\mathcal{A}$ is the subring of $D$ generated by $N_{>\alpha}$. The additional assumption that $D^{\times} / N$ satisfies Property $\left(3 \frac{1}{2}\right)$ is used only to show that in this case we can furthermore choose $\varphi$ so that the subgroup $\varphi\left(K^{\times}\right) \subseteq \Gamma$ is totally ordered (where $K$ is the center of $D$ and note that when $\operatorname{diam}\left(\Delta_{D^{\times} / N}\right) \geq 3$, $\left.K^{\times} \subseteq N\right)$. Then, together with Proposition 4.3 this implies that $\varphi$ satisfies all the hypotheses $(1)-(3)$ and $\left(4^{\prime}\right)$ of Theorem 2.3 of $\S 2$. This is proved in Theorem 5.8 at the end of this section.

We start by recalling some notation, definitions and preliminary results from $\S \S 1-3$ of 23 and $\delta \S 6-7$ of $\left[20\right.$. For an element $x \in D^{\times}$, we let $x^{*}$ denote its image in $G^{*}:=D^{\times} / N$. In addition $\Delta=\Delta_{D^{\times} / N}$ is the commuting graph of $D^{\times} / N$, and $d($,$) is the distance function in \Delta$.

$$
\text { For } x \in D^{\times} \text {, we let } N(x)=\{n \in N \mid x+n \in N\} \text {. }
$$

We recall that $\emptyset \varsubsetneqq N(x) \varsubsetneqq N$, for any $x \in D^{\times} \backslash N$ (Lemma 1.8 in 23]). For $x \in D^{\times} \backslash N$ we define the relation $\mathfrak{P}_{x^{*}}$ on $N$ by

$$
m \mathfrak{P}_{x^{*}} n \quad \Leftrightarrow \quad N(m x) \subseteq N(n x) \quad \text { for } m, n \in N
$$

(cf. $\S 6$ in $[20]$ ). The relation $\mathfrak{P}_{x^{*}}$ is independent of the coset representative $x$, and is a preorder relation compatible with the group structure (Lemma 6.4 in [20]). It follows that

$$
U_{x^{*}}=\{n \in N \mid N(n x)=N(x)\}
$$


is a normal subgroup of $N$. This yields the partially ordered group $\Gamma_{x^{*}}:=N / U_{x^{*}}$ with the order relation $\leq_{x^{*}}$ induced by $\mathfrak{P}_{x^{*}}$, and the (canonical) homomorphism $\varphi_{x^{*}}: N \rightarrow \Gamma_{x^{*}}$. Of course

$$
N_{\leq_{x^{*}} \alpha}=\left\{n \in N \mid \varphi_{x^{*}}(n) \leq \alpha\right\}, \quad \alpha \in \Gamma_{x^{*}},
$$

and $N_{>_{x^{*}} \alpha}, N_{\geq_{x^{*}} \alpha}$, etc. are defined similarly. Finally, let us recall the notation

$$
\mathbb{P}_{x^{*}}=\{a \in x N \mid 1 \in N(a)\},
$$

and that by $1.8(5)$ in $[23], \mathbb{P}_{x^{*}} \neq \emptyset$, for any $x \in D^{\times} \backslash N$.

Proposition 5.1. Let $x \in D^{\times} \backslash N$.

(1) For $n \in N \backslash N(x)$, the elements $x^{*}$ and $(x+n)^{*}$ commute in $G^{*}$, hence $d\left(x^{*},(x+n)^{*}\right) \leq 1$.

(2) Let $n \in N$ and $g \in D^{\times}$, then $N(n x)=n N(x), N(x n)=N(x) n$ and $N\left(x^{g}\right)=$ $g^{-1} N(x) g$ (where $\left.x^{g}=g^{-1} x g\right)$.

(3) If $n^{-1} \in N\left(x^{-1}\right)$, then $x+n \in x N$; in particular, $n \notin N(x)$.

(4) $N_{\leq_{x^{*}} 0}=\left\{n \in N \mid n \in N(a), \forall a \in \mathbb{P}_{x^{*}}\right\}$, in particular, $\varphi_{x^{*}}(m) \leq_{x^{*}} \varphi_{x^{*}}(n)$, iff $n a+m \in N$, for all $a \in \mathbb{P}_{x^{*}}$.

(5) If $a \in x N$ and $n \in N(a)$, then $\varphi_{x^{*}}(m) \leq_{x^{*}} \varphi_{x^{*}}(n)$ implies that $m \in N(a)$.

Proof. Part (1) is 2.1 in [23] and part (2) comes from 1.8(1) and 1.8(2) in [23]. Part (3) is immediate from the definitions. Parts (4) and (5) are Lemmas 6.5(1) and $6.5(3)$ in [20].

The next proposition gives some preliminary results in the case where $\operatorname{diam}(\Delta) \geq$ 3. Most of these results were already established in [20].

Proposition 5.2. Suppose $x, y \in D^{\times} \backslash N$ are such that $d\left(x^{*}, y^{*}\right) \geq 3$, and let $a \in x N, b \in y N$, and $\epsilon \in\{1,-1\}$. Then

(1) $a+b \notin N$ and $N(a+b)=N(a) \cap N(b)$.

(2) If $\epsilon \in N(a)$, then $N(b) \subseteq N(a b) \cap N(b a)$.

(3) If $\epsilon \in N\left(a^{-1}\right)$, then $N(a b) \cup N(b a) \subseteq N(b) \cap N(-b)$.

(4) If $1 \in N(a)$ then $1+a \in U_{y^{*}}$.

(5) If $1 \in N(a)$, then $N\left(a^{-1}\right) \subseteq N_{\leq_{y^{*}}}$, but $N\left(a^{-1}\right) \nsubseteq U_{y^{*}}$.

(6) $N_{<_{y^{*}}} \neq \emptyset$, and $N_{\leq_{y^{*} 0}} \nsubseteq K^{\times}$.

Proof. Parts (1), (2) and (3) are respectively Lemmas 6.8(1), 6.9(2) and 6.9(3), and (4) is a particular case of Lemma 7.2 in [20]. To prove (5), suppose $1 \in N(a)$ and let $c \in \mathbb{P}_{y^{*}}$. By (2) and (3) we have

$$
N\left(a^{-1}\right) \subseteq N\left(a^{-1} c\right) \subseteq N(c)
$$

As this holds for all $c \in \mathbb{P}_{y^{*}}$, Proposition 5.1(4) implies that $N\left(a^{-1}\right) \subseteq N_{\leq_{y^{*}} 0}$. Next, by (3) $N\left(a^{-1} c^{-1}\right) \subseteq N\left(a^{-1}\right) \cap N\left(c^{-1}\right)$, so for $n \in N\left(a^{-1} c^{-1}\right)$ we have $n \in$ $N_{\leq_{y^{*}} 0}$, and $n \in N\left(c^{-1}\right)$. By Proposition 5.1 (3), $n^{-1} \notin N(c)$, so $n^{-1} \notin U_{y^{*}}$, and it follows that $n \notin U_{y^{*}}$. The first part of (6) follows from (5). For the second part assume $1 \in N(a) \cap N(b)$. As in the proof of $(5), N\left(a^{-1} b^{-1}\right) \subseteq N_{<_{y^{*}} 0}$. By [6] or [33], there exist $n, m \in N$ such that $a^{-1} b^{-1}=n-m$. But since $K^{\times} \subseteq N$, we cannot have $m, n \in K$. Note however that $m,-n \in N\left(a^{-1} b^{-1}\right)$, so we see that $N\left(a^{-1} b^{-1}\right) \nsubseteq K$. 
Proposition 5.3. Let $x, y \in D^{\times} \backslash N$ with $d\left(x^{*}, y^{*}\right) \geq 3$. Pick $a \in \mathbb{P}_{x^{*}}, b \in \mathbb{P}_{y^{*}}$ and set $z=a^{-1} b^{-1}$. Then

(1) For $n \in N\left(a^{-1} b^{-1}\right)$ we have $(N(a) \cap N(b)) \pm n^{-1} \subseteq N(a) \cap N(b)$.

(2) For all $n \in N(z)$ we have $1 \pm n^{-1} \in N_{z_{z^{*}} 0}$.

(3) $N(z) \subseteq N_{\leq_{z^{*}} 0}$, and for any $m \in N(z)$ the element $\varphi_{z^{*}}\left(m^{-1}\right)$ is an s-level of $\varphi_{z^{*}}$

(4) $\varphi_{y^{*}}\left(\right.$ and $\left.\varphi_{x^{*}}\right)$ possesses an s-level.

Proof. (1) Note that by Proposition $5.2(3)$, for $\nu= \pm 1$ we have $N\left(a^{-1} b^{-1}\right) \subseteq$ $N\left(\nu a^{-1}\right) \cap N\left(\nu b^{-1}\right)$. Now let $n \in N\left(a^{-1} b^{-1}\right)$; then

$$
a+b=\left(a+n^{-1}\right)+\left(b-n^{-1}\right) .
$$

However, since $\pm n \in N\left(a^{-1}\right) \cap N\left(b^{-1}\right)$, we have $a+n^{-1} \in a N$ and $b-n^{-1} \in b N$ (Proposition 5.1(3)). Thus using Proposition 5.2(1) we get $N(a+b)=N\left(a+n^{-1}\right) \cap$ $N\left(b-n^{-1}\right)$. Using $a+b=\left(a-n^{-1}\right)+\left(b+n^{-1}\right)$ and arguing similarly we get that $N(a+b)=N\left(a-n^{-1}\right) \cap N\left(b+n^{-1}\right)$. So,

$$
N(a+b)=N\left(a+\epsilon n^{-1}\right) \cap N\left(b-\epsilon n^{-1}\right), \quad \text { for } \epsilon \in\{1,-1\} .
$$

Now let $m \in N(a) \cap N(b)=N(a+b)$. Then $a+\epsilon n^{-1}+m \in N \ni b+\epsilon n^{-1}+m$, so if $\epsilon n^{-1}+m \notin N$, then by Proposition [5.11 (1), $a^{*},\left(\epsilon n^{-1}+m\right)^{*}, b^{*}$ is a path in $\Delta$, contradicting $d\left(a^{*}, b^{*}\right) \geq 3$. This shows that $m \pm n^{-1} \in N(a) \cap N(b)$.

(2) Let $n \in N(z)$. Since $1 \in N(a) \cap N(b)$, by (1) we have $1 \pm n^{-1} \in N$. Now let $c \in \mathbb{P}_{z^{*}}$. Since $d\left(x^{*}, z^{*}\right) \geq 3 \leq d\left(y^{*}, z^{*}\right)$, by Proposition 5.2 (4) we have $c+1 \in U_{x^{*}} \cap U_{y^{*}} \subseteq N(a) \cap N(b)$. By (1), $c+1 \pm n^{-1} \in N(a) \cap N(b)$. As this holds for all $c \in \mathbb{P}_{z^{*}}$, we get that $1 \pm n^{-1} \in N_{\leq_{z^{*}} 0}$ (Proposition 5.1(4)).

(3) Let $m \in N(z)$; then $m \in N\left(a^{-1}\right)$. Since $d\left(x^{*}, z^{*}\right) \geq 3$, it follows from Proposition $5.2(5)$ that $m \in N_{z_{z^{*}} 0}$. Set $\alpha=\varphi_{z^{*}}\left(m^{-1}\right)$ and note that $0 \leq_{z^{*}}$ $\alpha \in \Gamma_{z^{*}}$. Let $n \in N_{z_{z^{*}} \alpha}$; then $\varphi_{z^{*}}\left(n^{-1}\right)<_{z^{*}} \varphi_{z^{*}}(m)$, so by Proposition 5.1 (5), $n^{-1} \in N(z)$. By part (2), $1 \pm n \in N_{\leq_{z^{*} 0}}$, so $\alpha$ is an s-level of $\varphi_{z^{*}}$ (see Definition 4.1).

(4) What we saw in (3) in fact is that if $d\left(r^{*}, s^{*}\right) \geq 3$, then $\varphi_{\left(r^{-1} s^{-1}\right)^{*}}$ possesses an s-level. So taking $r=x^{-1}$ and $s=y^{-1} x$ we are done.

Proposition 5.4. Let $x, y \in D^{\times} \backslash N$ with $d\left(x^{*}, y^{*}\right) \geq 3$. Let $0 \leq \alpha \in \Gamma_{y^{*}}$ be an s-level of $\varphi_{y^{*}}$, and let $\mathcal{A}$ be the subring of $D$ generated by $N_{>_{y^{*}} \alpha}$. Suppose $a \in \mathbb{P}_{x^{*}}, b \in \mathbb{P}_{y^{*}}$ and pick $m \in N\left(a^{-1} b^{-1}\right)$. Then for $\mu>_{y^{*}} \varphi_{y^{*}}\left(m^{-1}\right)$, we have $0 \leq_{y^{*}} \mu \in \Gamma_{y^{*}}$ and $\mathcal{A} \cap N \subseteq N_{>_{y^{*}}-\mu}$.

Proof. First we claim that

$$
N\left(a^{-1} b^{-1}\right) \pm 1 \subseteq N\left(a^{-1} b^{-1}\right) .
$$

Indeed, set $z=a^{-1} b^{-1}$ and let $n \in N(z)$. By Proposition 5.3 (2),

$$
n^{-1}(n \pm 1)=1 \pm n^{-1} \in N_{\leq_{z^{*}} 0},
$$

which means that $\varphi_{z^{*}}(n \pm 1) \leq_{z^{*}} \varphi_{z^{*}}(n)$. By Proposition 5.1(5), as $n \in N(z)$, also $n \pm 1 \in N(z)$ and (i) is proved.

Set $\leq=\leq_{y^{*}}, \varphi=\varphi_{y^{*}}$, and let $m \in N(z)$. As $m \in N\left(a^{-1}\right)$, Proposition 5.2 (5) implies that $m \in N_{\leq 0}$, so $\mu>0$. Thus to complete the proof it suffices to show that

$$
\mathcal{A} \cap N \subseteq N_{\geq \varphi(m)}
$$


Let $n \in \mathcal{A} \cap N$. We must show that $\varphi(n) \geq \varphi(m)$, i.e. that $n t+m \in N$, for all $t \in \mathbb{P}_{y^{*}}$ (see Proposition 5.1(4)). We have

$$
n t+m=(n+1) t+(-t+m) \text {. }
$$

Now,

$$
-t+m=(1+t)\left(-1+(1+t)^{-1}(1+m)\right) \in N\left(a^{-1} b^{-1}\right) .
$$

Indeed $t+1 \in U_{\left(a^{-1} b^{-1}\right)^{*}}$ by Proposition 5.2(4), and $1+m \in N\left(a^{-1} b^{-1}\right)$ by (ii). It follows (using also Proposition 5.1 (2)) that $(1+t)^{-1}(1+m) \in N\left(a^{-1} b^{-1}\right)$, and then by (1i) again $-1+(1+t)^{-1}(1+m) \in N\left(a^{-1} b^{-1}\right)$ validating (iv). Now, we see from (iv) that $-t+m \in N\left(a^{-1} b^{-1}\right) \subseteq N\left(a^{-1}\right)$. In particular, by Proposition 5.2(5), $-t+m \in N_{\leq 0}$. On the other hand, by Proposition 4.2(2) we have $n+1 \in$ $\operatorname{Ker} \varphi=U_{y^{*}}$, and therefore $(n+1) t \in \mathbb{P}_{y^{*}}$. Hence, by (iii) and Proposition 5.1 (4), $n t+m \in N$, completing the proof of (iii) and of the proposition.

We now turn to the proof of the fact that (after choosing the element $y$ properly) the subgroup $\varphi_{y^{*}}\left(K^{\times}\right) \subseteq \Gamma_{y^{*}}$ is totally ordered. This does not follow from the hypothesis that $d\left(x^{*}, y^{*}\right) \geq 3$, because our arguments in effect show that the fact that $\varphi_{y^{*}}\left(K^{\times}\right)$is totally ordered implies that $N$ is open with respect to a single valuation. This is not generally true when $d\left(x^{*}, y^{*}\right)=3$ (see Example 8.4 in [20]). Hence we will need the following crucial additional hypothesis.

Property $\left(3 \frac{1}{2}\right)$. Given a finite group $H$ and elements $c, d$ in the commuting graph $\Delta_{H}$ of $H$ (having distance function $d_{H}($,$) ), we will say that (H, c, d)$ satisfies Property $\left(3 \frac{1}{2}\right)$, if for every $a, b \in \Delta_{H}$ such that $[c, a]=1=[d, b]$, there exists $h \in H$ satisfying $d_{H}\left(c^{h}, d\right) \geq 3$ and $\left[a^{h}, b\right] \neq 1$. (When the group $H$ is understood from the context, we will just say that $c, d$ satisfy Property $\left(3 \frac{1}{2}\right)$.)

As we mentioned in the introduction, a crucial point in the proof of the Main Theorem is that Property $\left(3 \frac{1}{2}\right)$ holds true for all minimal nonsolvable groups (see $\S 7)$. To derive the required fact that the group $\varphi_{y^{*}}\left(K^{\times}\right)$is totally ordered from Property $\left(3 \frac{1}{2}\right)$, we need some additional notation.

Notation 5.5. (1) For $r \in D^{\times} \backslash N$ we let $\dot{N}(r)=N(r) \cap K$. (Note that although $N(r)$ is always nonempty, $\dot{N}(r)$ may well be empty.)

(2) For $r, s \in D^{\times} \backslash N$, we define $\operatorname{In}_{K}\left(r^{*}, s^{*}\right)$ and $\operatorname{Inc}_{K}\left(r^{*}, s^{*}\right)$ as we define $\operatorname{In}($, and $\operatorname{Inc}($,$) in [23, p. 228, using \dot{N}()$ instead of $N(): \operatorname{In}_{K}\left(r^{*}, s^{*}\right)$ means that for all pairs $(a, b) \in(r N \times s N)$, either $\dot{N}(a) \subseteq \dot{N}(b)$ or $\dot{N}(b) \subseteq \dot{N}(a)$, and $\operatorname{Inc}_{K}\left(r^{*}, s^{*}\right)$ means that $\operatorname{In}_{K}\left(r^{*}, s^{*}\right)$ holds and, in addition, given $c \in \mathbb{P}_{r^{*}}$, there exists $d \in \mathbb{P}_{s^{*}}$ with $\dot{N}(c) \supseteq \dot{N}(d)$.

For the sake of completeness we include here the following lemma which is actually part of Lemma 6.12 in [20].

Lemma 5.6. Let $x, y \in D^{\times} \backslash N$. Then

(1) If $\operatorname{In}_{K}\left(x^{*}, y^{*}\right)$, then either $\operatorname{Inc}_{K}\left(x^{*}, y^{*}\right)$ or $\operatorname{Inc}_{K}\left(y^{*}, x^{*}\right)$.

(2) If $\operatorname{Inc}_{K}\left(y^{*}, x^{*}\right)$, then $\operatorname{In}_{K}\left(y^{*}, y^{*}\right)$.

Proof. Suppose $\operatorname{Inc}_{K}\left(x^{*}, y^{*}\right)$ does not hold. Then there is $a \in \mathbb{P}_{x^{*}}$ such that $\dot{N}(a) \subseteq$ $\dot{N}(b)$, for all $b \in \mathbb{P}_{y^{*}}$, so $\operatorname{Inc}_{K}\left(y^{*}, x^{*}\right)$ holds, proving (1). Now, assume $\operatorname{Inc}_{K}\left(y^{*}, x^{*}\right)$ and let $b, c \in y N$ be such that $\dot{N}(b) \nsubseteq \dot{N}(c)$. Pick $n \in \dot{N}(b), n \notin \dot{N}(c)$. Then $\dot{N}\left(n^{-1} b\right) \ni 1 \notin \dot{N}\left(n^{-1} c\right)$ (we note that $n \in K^{\times}$, so $\dot{N}\left(n^{-1} c\right)=n^{-1} \dot{N}(c)$, for any 
$\left.c \in D^{\times} \backslash N\right)$. By $\operatorname{Inc}_{K}\left(y^{*}, x^{*}\right)$, we can pick $a \in \mathbb{P}_{x^{*}}$ with $\dot{N}\left(n^{-1} b\right) \supseteq \dot{N}(a)$. Since $1 \notin \dot{N}\left(n^{-1} c\right)$, the inclusion $\dot{N}(a) \subseteq \dot{N}\left(n^{-1} c\right)$ is impossible, so by $\operatorname{In}_{K}\left(x^{*}, y^{*}\right)$ we have $\dot{N}\left(n^{-1} c\right) \subseteq \dot{N}(a) \subseteq \dot{N}\left(n^{-1} b\right)$, hence $\dot{N}(c) \subseteq \dot{N}(b)$, as required.

Proposition 5.7. Let $x^{*}, y^{*} \in D^{\times} / N$ be such that $d\left(x^{*}, y^{*}\right) \geq 3$ and suppose Property $\left(3 \frac{1}{2}\right)$ holds for $x^{*}, y^{*}$. Then

(1) $\operatorname{In}_{K}\left(x^{*}, y^{*}\right)$ holds.

(2) After perhaps interchanging $x^{*}, y^{*}$, we have that the subgroup $\varphi_{y^{*}}\left(K^{\times}\right) \subseteq \Gamma_{y^{*}}$ is totally ordered.

Proof. (1) Let $c \in x N$ and $d \in y N$ be such that $\dot{N}(c) \nsubseteq \dot{N}(d)$ and $\dot{N}(d) \nsubseteq \dot{N}(c)$. Picking $n \in \dot{N}(c) \backslash \dot{N}(d)$ and replacing $c, d$ with $-n^{-1} c,-n^{-1} d$, we may assume that $-1 \in \dot{N}(c) \backslash \dot{N}(d)$. By our assumption, there exists $k \in \dot{N}(d) \backslash \dot{N}(c)$. Now of course

$$
\left[c^{*},(c+k)^{*}\right]=\left[(d-1)^{*}, d^{*}\right]=1,
$$

and since by hypothesis $c^{*}, d^{*}$ satisfy Property $\left(3 \frac{1}{2}\right)$, there exists $g \in D^{\times}$such that $d\left(\left(c^{g}\right)^{*}, d^{*}\right) \geq 3$ and $\left((c+k)^{g}\right)^{*}=\left(c^{g}+k\right)^{*}$ does not commute with $(d-1)^{*}$. Note however that $-1 \in N\left(c^{g}\right)$ and it follows from Proposition [5.2 2 ) that $k \in N(d) \subseteq$ $N\left(d c^{g}\right)$. In view of Proposition 5.1 (1) and the fact that $d c^{g}+k \in N$, we have

$$
\begin{aligned}
{\left[\left(c^{g}+k\right)^{*},(d-1)^{*}\right] } & =\left[d^{*}\left(c^{g}+k\right)^{*},(d-1)^{*}\right] \\
& =\left[\left(\left(d c^{g}+k\right)+(d-1) k\right)^{*},((d-1) k)^{*}\right]=1,
\end{aligned}
$$

a contradiction, proving (1).

(2) By (1), $\operatorname{In}_{K}\left(x^{*}, y^{*}\right)$ holds, so by Lemma 5.6(1), we may assume (after perhaps interchanging $x^{*}$ and $\left.y^{*}\right)$ that $\operatorname{Inc}_{K}\left(y^{*}, x^{*}\right)$ holds. By Lemma 5.6(2), $\operatorname{In}_{K}\left(y^{*}, y^{*}\right)$ holds. Let $k \in K^{\times}$and assume $k \notin N_{\leq_{y^{*}} 0}$. Then there exists $b \in y N$ with $k \notin N(b) \ni 1$ (see Proposition 5.1(4)), and consequently $1 \notin N\left(k^{-1} b\right) \ni k^{-1}$. From $\operatorname{In}_{K}\left(y^{*}, y^{*}\right)$, we see that $k^{-1} \in N(s)$, for all $s \in \mathbb{P}_{y^{*}}$, so $k^{-1} \in N_{\leq_{y^{*}}}$. This means that the subgroup $\varphi_{y^{*}}\left(K^{\times}\right) \subseteq \Gamma_{y^{*}}$ is totally ordered.

The following theorem summarizes the results of $\S \S 4-5$.

Theorem 5.8. Let $y \in D^{\times} \backslash N$ be an element for which there exists $x \in D^{\times} \backslash N$ with $d\left(x^{*}, y^{*}\right) \geq 3$. Set $\varphi=\varphi_{y^{*}}, \geq=\geq_{y^{*}}$, and $\Gamma=\Gamma_{y^{*}}$. Then

(1) $\Gamma$ is nontrivial and $N_{\geq 0} \nsubseteq K^{\times}$.

(2) $\varphi$ is a strongly leveled map.

(3) If $\mathcal{R} \subseteq D$ is the subring generated by $N_{\geq 0}$, then $\mathcal{R} \cap N \subseteq N_{>-\gamma}$ for some $\gamma \in \Gamma_{\geq 0}$.

Furthermore, if $x^{*}, y^{*}$ satisfy Property $\left(3 \frac{1}{2}\right)$, then after interchanging $x^{*}$ and $y^{*}$ if necessary we have that $\Gamma_{K}=\varphi\left(K^{\times}\right)$is a nontrivial totally ordered subgroup of $\Gamma$ and that $\varphi$ possesses an s-level in $\left(\Gamma_{K}\right)_{\geq 0}$.

Proof. Part (1) follows from Proposition 5.2(6) and part (2) is Proposition 5.3(4). Part (3) is a consequence of Proposition 5.4 and Proposition 4.3(3). Assume that $x^{*}, y^{*}$ satisfy Property $\left(3 \frac{1}{2}\right)$. Then by Proposition $5.7(2)$, after perhaps interchanging $x^{*}$ and $y^{*}$, we have that $\Gamma_{K}$ is totally ordered, so Proposition 4.3 (2) completes the proof. 


\section{THE MAIN THEOREM: PROOF AND APPLICATIONS}

Combining Theorem 2.3 and Theorem [5.8, we obtain the following, which is Theorem 1 of the introduction.

Theorem 6.1. Let $D$ be a finite dimensional division algebra over a finitely generated infinite field, and let $N \subseteq D^{\times}$be a normal subgroup of finite index. If $D^{\times} / N$ satisfies Property $\left(3 \frac{1}{2}\right)$, then $N$ is open in $D^{\times}$with respect to a nontrivial height one valuation of $D$.

Indeed, observe that if $x^{*}, y^{*} \in D^{\times} / N$ satisfy Property $\left(3 \frac{1}{2}\right)$, then we may assume without loss of generality that $d_{D \times / N}\left(x^{*}, y^{*}\right) \geq 3$. So Theorem 5.8 applies and yields the homomorphism $\varphi_{y^{*}}: N \rightarrow \Gamma_{y^{*}}$ satisfying all hypotheses (1) - (3) and $\left(4^{\prime}\right)$ of Theorem 2.3 . So Theorem 2.3 completes the proof.

Another ingredient in the proof of our Main Theorem is the Nonexistence Theorem stated in the introduction. For the reader's convenience we give here a brief summary of its proof, referring to [20] for the details.

Sketch of the proof of the Nonexistence Theorem. Suppose the conclusion of the theorem is false, and let $H \in \mathcal{G}$ be a member of minimal possible order which is a quotient of the multiplicative group of some finite dimensional central division algebra. Consider hereafter only finite dimensional division algebras $D$ with center $K$ such that $H$ is a quotient of $D^{\times}$. If there exists such a $D$ having a positive characteristic, we will consider only division algebras in this positive characteristic; otherwise, all algebras considered will have characteristic zero. One shows that there is a division algebra $D$ satisfying all the above requirements and also such that $K$ is finitely generated; then, in particular, the transcendence degree $t=\operatorname{tr} \cdot \operatorname{deg}_{K_{0}} K$ is finite. This allows us to pick from among all division algebras singled out in the previous steps an algebra $D$ for which the corresponding transcendence degree $t$ is (finite and) minimal (we observe that, due to Wedderburn's theorem, $t>0$ if char $>0$ ). By our construction, there exists a surjective homomorphism $D^{\times} \rightarrow H$, and we let $N$ denote its kernel. According to condition (4) in the statement of the Nonexistence Theorem, $N$ is open in $D^{\times}$with respect to a nontrivial height one valuation of $D$ as $H$ was chosen to be a minimal member of $\mathcal{G}$.

Let $\bar{D}:=\bar{D}_{v}$ be the residue division algebra. One shows then that the openness of $N$ implies the existence of a normal subgroup $\bar{N} \subseteq \bar{D}^{\times}$such that the following holds: There exists $M_{1} \triangleleft H_{1} \triangleleft H$ with $M_{1}$ and $H / H_{1}$ solvable, and $\bar{D}^{\times} / \bar{N} \cong H_{1} / M_{1}$. However, conditions (1) - (3) together with the minimality of $H$ imply that $H_{1}=H$ and $M_{1}=\{1\}$, i.e. $H$ will be a quotient of $\bar{D}^{\times}$as well. Then our choice of the characteristic of $D$ implies that $\bar{D}_{v}$ has the same characteristic as $D$, and it follows from a result in the theory of valuations that the transcendence degree of the center of $\bar{D}_{v}$ over its prime subfield will be strictly less than $t$, a contradiction, proving the Nonexistence Theorem.

Now, the class $\mathcal{G}=\mathcal{N S}$ of all nonsolvable groups obviously satisfies conditions (1) - (3) of the Nonexistence Theorem. Furthermore, by Theorem 2 of the introduction, which will be proved in \$7] minimal members of $\mathcal{N S}$ satisfy Property $\left(3 \frac{1}{2}\right)$. In conjunction with Theorem 6.1 this implies that condition (4) holds for $\mathcal{N S}$ as well. Thus, applying the Nonexistence Theorem we obtain

Main Theorem. Let $D$ be a finite dimensional division algebra. Then any finite quotient of the multiplicative group $D^{\times}$is solvable. 
The rest of the section is devoted to applications of the Main Theorem primarily to the normal subgroup structure of the group $S L(1, D)$ of elements in $D^{\times}$having reduced norm one.

Corollary 6.2. Let $N \subseteq S L(1, D)$ be a subgroup of finite index which is normal in $D^{\times}$. Then the quotient $S L(1, D) / N$ is solvable.

Proof. The group $D^{\times}$acts on $S L(1, D) / N=: B$ by conjugation, and we let $M$ denote the kernel of this action. Then

$$
B / Z(B) \simeq \operatorname{Int} B \hookrightarrow D^{\times} / M .
$$

But the group $D^{\times} / M$ is finite (as a subgroup of the automorphism group of the finite group $B$ ), hence solvable by the Main Theorem, and the solvability of $B$ follows.

Here is one case where Corollary 6.2 gives the solvability of all finite quotients of $S L(1, D)$.

Corollary 6.3. Let $D$ be a finite dimensional division algebra over a global field $K$. Then any finite quotient of $S L(1, D)$ is solvable.

Proof. Let $N \subseteq S L(1, D)$ be a normal subgroup of a finite index $m$. We denote by $N_{0}$ the subgroup generated by the elements $g^{m}, g \in S L(1, D)$. Then $N_{0} \subseteq N$ and $N_{0} \triangleleft D^{\times}$. Since $S L(1, D)$ does not have finite exponent (this follows from its Zariski density in the corresponding algebraic group $\mathbf{S L}_{1, D}$ which is isomorphic to $\mathbf{S L}_{n}$ over the algebraic closure of $\left.K\right), N_{0}$ is noncentral and therefore, by a theorem 3 due to Margulis and Prasad (see [13, [17]), $N_{0}$ has a finite index in $S L(1, D)$. By Corollary6.2 the quotient $S L(1, D) / N_{0}$ is solvable, and then so is $S L(1, D) / N$.

Remark. In view of the Margulis-Prasad theorem, when $K$ is a global field, Corollary 6.3 in effect yields the solvability of any quotient of $S L(1, D)$ by a noncentral normal subgroup (cf. [26], Thm. 12.4).

The question of whether Corollary 6.3 extends to division algebras over arbitrary fields remains open (we note that this is exactly Question 2 of the introduction for the algebraic group $G=\mathbf{S L}_{1, D}$ associated with $S L(1, D)$ ). Obviously, for the affirmative answer it would be sufficient to show that any finite index normal subgroup $N \subseteq S L(1, D)$ contains a finite index subgroup $N_{0} \subseteq S L(1, D)$ which is normal in $D^{\times}$. In other words, one needs to show that $\bigcap_{g \in D^{\times}}\left(g N g^{-1}\right)$ has finite index in $S L(1, D)$, or equivalently, that there are only finitely many distinct conjugates $g N g^{-1}, g \in D^{\times}$. In this regard, we observe that one of the ingredients of the proof of the Margulis-Prasad theorem is the fact that if $G$ is an absolutely simple simply connected algebraic group over a global field $K$, then there exists an $S$-arithmetic subgroup $\Gamma \subseteq G(K)$, for a sufficiently large finite set of places $S$, such that $\Gamma N=G(K)$, for any noncentral normal subgroup $N \subseteq G(K)$, implying $G(K) / N \simeq \Gamma /(\Gamma \cap N)$. If char $K=0$, then $\Gamma$ is always finitely generated (cf. [15], Theorem 5.11), and if char $K>0$, then one can enlarge $S$ to make $\Gamma$ finitely generated (cf. Behr [4]). Then $\Gamma$, and therefore also $G(K)$, has only finitely many homomorphisms to any given finite group. In the set-up above, this gives the finiteness of the number of distinct conjugates $g N g^{-1}, g \in D^{\times}$. To what extent

\footnotetext{
${ }^{3}$ This theorem states that if $G$ is an absolutely simple simply connected algebraic group over a global field $K$, then any noncentral normal subgroup $N \subseteq G(K)$ has finite index.
} 
this kind of an argument can be generalized to arbitrary (finitely generated) fields remains to be seen.

We now turn to the Margulis-Platonov conjecture (conjecture (MP)). We refer the reader to $\mathrm{Ch}$. IX in [15] and Appendix A in [20] for a detailed discussion of (MP). The proof of (MP) for $G=\mathbf{S L}_{1, D}$ was recently completed in 23] and [26], using a reduction obtained in [19]. Our Main Theorem, however, enables one to give a short proof of (MP) for $G=\mathbf{S L}_{1, D}$ which does not require the reduction of [19].

Theorem 6.4 ([26], Thm. 2). Let $D$ be a division algebra over a global field $K$. Then the group $G=\mathbf{S L}_{1, D}$ satisfies the Margulis-Platonov conjecture.

Proof. We recall that (MP) for the group $G=\mathbf{S L}_{1, D}$ is equivalent to the statement that if $T$ is the (finite) set of all nonarchimedean places $v$ of $K$ for which $D \otimes_{K} K_{v}$ is a division algebra, then for any noncentral normal subgroup $N \subseteq G(K)=$ $S L(1, D)$ there exists an open normal subgroup $W \subseteq \prod_{v \in T} G\left(K_{v}\right)=: G_{T}$ such that $N=G(K) \cap \delta^{-1}(W)$, where $\delta: G(K) \rightarrow G_{T}$ is the diagonal embedding if $T \neq \emptyset$, and $\delta$ is the trivial map otherwise. In other words, $N$ is open in $G(K)$ with respect to the topology defined by the valuations in $T$ (which is sometimes called the $T$-adic topology). It was first proved in [16] (cf. also [15], §9.2) that if $K$ is a number field, then $N=[S L(1, D), S L(1, D)]$ is $T$-adically open. This fact was extended to arbitrary global fields by Raghunathan [18 who proved the following more general result: if $U \subseteq S L(1, D)$ is a $T$-adically open subgroup, then its commutator subgroup $[U, U]$ is also $T$-adically open. In particular, this implies that any term of the derived series for $S L(1, D)$ is $T$-adically open. Now, given an arbitrary noncentral normal subgroup $N \subseteq S L(1, D)$, it has finite index by the Margulis-Prasad theorem, and then the quotient $S L(1, D) / N$ is solvable by Corollary 6.3 above. This implies that $N$ contains some term of the derived series of $S L(1, D)$, and the openness of $N$ follows.

\section{Property ( $\left.3 \frac{1}{2}\right)$ For minimal nonsolvable groups}

The purpose of this section is to prove Theorem 2 of the introduction, i.e. to show that minimal nonsolvable groups satisfy Property $\left(3 \frac{1}{2}\right)$ of the introduction. Recall that for any group $H$, the commuting graph of $H$ is the graph whose vertex set is $H \backslash\{1\}$ and whose edges are commuting pairs of elements. We denote by $\Delta_{H}$ the commuting graph of $H$, and by $d_{H}($, ) the usual distance function on $H$. So for $x, y \in \Delta_{H}, d_{H}(x, y)$ is the minimal number of edges in a path from $x$ to $y$ in $\Delta_{H}$ (it is $\infty$ if no such path exists).

Notation 7.1. Let $\Delta$ be a graph with distance function $d($,$) and let x, y \in \Delta$.

(1) $\Delta(x):=\{y \in \Delta \mid d(x, y)=1\}$.

(2) Given an integer $i \geq 1$, we denote $\Delta^{\leq i}(x)=\{y \in \Delta \mid d(x, y) \leq i\}$. The set $\Delta^{\geq i}(x)$ is defined similarly.

(3) Let $H$ be a group. For $c, d \in H$, we let $c^{d}=d^{-1} c d,[c, d]=c^{-1} d^{-1} c d$ and $c^{H}$ is the conjugacy class of $c$ in $H$.

A minimal nonsolvable group is a finite group $G$ such that $G$ is not solvable, but $G / M$ is solvable for every $1 \neq M \triangleleft G$. Throughout this section the following property of a finite group $H$ will be considered. 
Property $\left(3 \frac{1}{2}\right)$. There are two elements $c, d \in \Delta_{H}$ having the following property: There exists a subgroup $H_{1} \subseteq H$ such that for every $a \in \Delta_{H}(c)$ and $b \in \Delta_{H}(d)$, there exists $h \in \bar{H}_{1}$ satisfying $d_{H}\left(c^{h}, d\right) \geq 3$ and $\left[a^{h}, b\right] \neq 1$.

When a group $H$ satisfies Property $\left(3 \frac{1}{2}\right)$ with some elements $c, d \in \Delta_{H}$, where $H_{1}=H$, we will just say that $H$ satisfies Property $\left(3 \frac{1}{2}\right)$; or $(H, c, d)$ satisfies Property $\left(3 \frac{1}{2}\right)$, when we want to emphasize the elements $c, d$. When we want to emphasize the subgroup $H_{1}$ and the elements $c$, $d$, we will write $\left(H, c, d, H_{1}\right)$ satisfies Property $\left(3 \frac{1}{2}\right)$ (or $\left(H, c, d, H_{1}\right)$ do not satisfy Property $\left(3 \frac{1}{2}\right)$ ).

The purpose of this section is to prove the following theorem.

Theorem 7.2. Let $G$ be a minimal nonsolvable group. Then $G$ satisfies Property $\left(3 \frac{1}{2}\right)$.

One may think of Property $\left(3 \frac{1}{2}\right)$ as a property distinguishing a minimal nonsolvable group $G$ from a direct product (of more than one group), in terms of the commuting graph. As the reader will notice, if $G$ is a direct product, then $G$ does not have Property $\left(3 \frac{1}{2}\right)$, and indeed, this may be thought of as a "reason" that certain direct products can be quotients of the multiplicative group of $D$ while other groups ("close" to being wreath products, such as minimal nonsolvable groups) cannot.

Notation 7.3. Throughout this section, $G$ is a minimal nonsolvable group. Recall that $G$ has a unique minimal normal subgroup $K$ such that there exists a subgroup $L \leq K$ satisfying: $L$ is a nonabelian simple group and $K$ is the direct product of the distinct conjugates $L^{g_{1}}, \ldots, L^{g_{n}}$ of $L, n \geq 1$. Furthermore, $G$ acts transitively by conjugation on the set $\left\{L^{g_{1}}, \ldots, L^{g_{n}}\right\}, G / K$ is solvable and $C_{G}(K)=1$. The notation $K, L$ and $g_{1}, \ldots, g_{n}$ are fixed throughout this section. We further fix $L_{i}$ to denote $L^{g_{i}}, 1 \leq i \leq n$, and we assume $L_{1}=L$. Given an element $x \in K$, $x_{i} \in L_{i}(1 \leq i \leq n)$, denote the elements so that $x=x_{1} \cdots x_{n}$. Note that when $n=1, G$ is an almost simple group, i.e. $L \subseteq G \subseteq \operatorname{Aut}(L)$.

In subsection 7.1 below, we will establish some further notation for $G$ and other notation to be used throughout this section. Then we will formulate certain conditions on $L$ that imply Theorem 7.2 These conditions are given in Proposition 7.1.8. We formulate the generic condition separately (see (a) of Proposition 7.1.8).

Proposition 7.4. Assume that there exist six distinct nonidentity conjugacy classes $B, C_{1}, \ldots, C_{5}$ of $\operatorname{Aut}(L)$ contained in $L$, such that $d_{\mathrm{Aut}(L)}(r, s) \geq 3$, for all $r \in B$ and $s \in \bigcup_{i=1}^{5} C_{i}$. Then $G$ satisfies Property $\left(3 \frac{1}{2}\right)$.

Subsection 7.2 is devoted to proving

Theorem 7.5. Let $L$ be a nonabelian finite simple group. Then $L$ contains six distinct nonidentity $\operatorname{Aut}(L)$-conjugacy classes $B, C_{1}, \ldots, C_{5}$ such that $d_{\operatorname{Aut}(L)}(r, s) \geq$ 3, for all $r \in B$ and $s \in \bigcup_{i=1}^{5} C_{i}$, provided $L$ is not isomorphic to one of the following groups:

$$
\operatorname{PSL}(2, q), q=5,7,8,9,11,16 \text { or } 27, \quad \operatorname{PSL}(3,4) \text { or } \operatorname{PSO}^{+}(8,2) \text {. }
$$

It follows from Theorem[7.5 and Proposition 7.4 that if $L$ is not one of the nine exceptional cases of Theorem [7.5, then $G$ satisfies Property $\left(3 \frac{1}{2}\right)$. The purpose of the final subsection $[7.3$ is to show that in the nine exceptional cases $G$ also satisfies Property $\left(3 \frac{1}{2}\right)$ and this is done using the results of subsection 7.1 
7.1. Conditions on $L$ that guarantee Property $\left(3 \frac{1}{2}\right)$. Throughout $\$ 7$ we let

$$
X:=\left\{L^{g_{1}}, \ldots, L^{g_{n}}\right\}
$$

and

$$
\begin{aligned}
& \Sigma \subseteq \operatorname{Sym}(X) \text { is the permutation group on } X \\
& \text { induced from the conjugation action of } G \text {. }
\end{aligned}
$$

Of course $\Sigma$ is a solvable transitive permutation group on $X$. We mention that in Lemmas 7.1.1 7.1.2 and 7.1.3 and in the notation given in the next paragraph, we think of $X$ as an arbitrary finite set and we think of $\Sigma \subseteq \operatorname{Sym}(X)$ as an arbitrary transitive solvable permutation group. Otherwise $X$ and $\Sigma$ are as in (i) and (ii).

We will use notation as in $\S 2$ of [25. Thus given $\tau \in \operatorname{Sym}(X)$ and $Y \subseteq X$, $\tau(Y):=\{\tau(y) \mid y \in Y\}, \Sigma(Y):=\{\sigma \in \Sigma \mid \sigma(Y)=Y\}$ is the global stabilizer of $Y$ in $\Sigma, \Sigma_{Y}:=\{\sigma \in \Sigma \mid \sigma(y)=y, \forall y \in Y\}$ is the pointwise stabilizer of $Y$ in $\Sigma$, and $\Sigma^{Y}:=\Sigma(Y) / \Sigma_{Y}$ is the action of $\Sigma$ on $Y$.

We use similar notation for actions on partitions. A partition $\mathcal{P}$ of $X$ is a collection $\mathcal{P}:=\left\{V_{1}, \ldots, V_{k}\right\}$ of nonempty subsets of $X$ such that $V_{i}$ are pairwise disjoint and their union is $X$.

Given a partition $\mathcal{P}$ of $X$ we write $\Sigma(\mathcal{P})$ for the subgroup of all permutations $\sigma \in \Sigma$ such that $\sigma\left(V_{i}\right) \in \mathcal{P}$, for all $1 \leq i \leq k$. We write $\Sigma_{\mathcal{P}}$ for the subgroup of all permutations $\sigma \in \Sigma$ such that $\sigma\left(V_{i}\right)=V_{i}$, for all $1 \leq i \leq k$. Finally, write $\Sigma^{\mathcal{P}}=\Sigma(\mathcal{P}) / \Sigma_{\mathcal{P}}$

We fix the following notation, except in the case when $n=1$,

$$
\mathcal{M}:=\left\{X_{1}, \ldots, X_{m}\right\}(m \geq 1) \text { is a partition of } X \text { stabilized by } \Sigma
$$

$$
\text { (i.e. } \Sigma=\Sigma(\mathcal{M}) \text { ), such that }\left|X_{i}\right| \geq 2 \text { and such that } \Sigma^{X_{i}} \text { is primitive. }
$$

When $n=1$, we let $\mathcal{M}=\{L\}$. We will consider conjugacy classes $C$ of $\operatorname{Aut}(L)$ contained in $L$. All such conjugacy classes are automatically assumed to be nonidentity conjugacy classes. Given such a class $C \subseteq L, C^{g_{i}} \subseteq L_{i}$ denotes the corresponding $\operatorname{Aut}\left(L_{i}\right)$ conjugacy class, $1 \leq i \leq n$ (recall that $L_{i}, g_{i}$ are as in Notation 7.3).

In order to construct elements in $K$ satisfying various properties we need some information about actions of solvable groups on partitions. This is done in the next three lemmas. Before we start with our lemmas we mention that when we write $\Sigma^{X_{i}} \cong S_{4}$, it is assumed that $\left|X_{i}\right|=4$ (this is in fact implied since $\Sigma^{X_{i}}$ is faithful and primitive on $X_{i}$ ).

Lemma 7.1.1. Let $n \geq 2$ and let $\Sigma$ be a transitive solvable permutation group on a set $X$ of size $n$. Let $\mathcal{M}=\left\{X_{1}, \ldots, X_{m}\right\}(m \geq 1)$ be a partition of $X$ stabilized by $\Sigma$, such that $\left|X_{i}\right| \geq 2$. Then there exists a partition $\mathcal{Q}_{1}=\left\{V_{1}, \ldots, V_{e}\right\}$ of $X$, with $2 \leq e \leq 3$ such that for all $\sigma \in \Sigma, \Sigma_{\sigma\left(\mathcal{Q}_{1}\right)} \subseteq \Sigma_{\mathcal{M}}$

Proof. We will think of $\Sigma^{\mathcal{M}}$ as a permutation group on $\{1, \ldots, m\}$. Partition the set $\{1, \ldots, m\}$ into $f$ parts, $1 \leq f \leq 5, \mathcal{J}=\left\{J_{1}, \ldots, J_{f}\right\}$, such that $\Sigma_{\mathcal{J}}^{\mathcal{M}}=1$. The existence of the partition $\mathcal{J}$ is guaranteed by Theorem 1.2 in [27] (see also Proposition 2.5 in [25]). If $m=1$, then $\mathcal{Q}_{1}$ can be any partition of $X$. Hence we may assume that $m>1$ and then $f \geq 2$. Write $X_{i}=\left\{x_{i}^{1}, \ldots, x_{i}^{t}\right\} \quad$ so $\left|X_{i}\right|=t$ ). We define $V_{1}$ as

$$
V_{1}=\bigcup_{i \in J_{1}}\left\{x_{i}^{1}\right\} \cup \bigcup_{i \in J_{2}}\left\{x_{i}^{1}, x_{i}^{2}\right\} \cup \bigcup_{i \in J_{3}}\left\{x_{i}^{1}, x_{i}^{2}, x_{i}^{3}\right\},
$$


where $\cup \bigcup_{i \in J_{3}}\left\{x_{i}^{1}, x_{i}^{2}, x_{i}^{3}\right\}$ occurs if and only if $f>2$ and $t>2$. If $2 \leq f \leq 3$ we define

$$
V_{2}=\bigcup_{i \in J_{1}}\left(X_{i} \backslash\left\{x_{i}^{1}\right\}\right) \cup \bigcup_{i \in J_{2}}\left(X_{i} \backslash\left\{x_{i}^{1}, x_{i}^{2}\right\}\right) \cup \bigcup_{i \in J_{3}} Y_{i},
$$

where $\cup \bigcup_{i \in J_{3}} Y_{i}$ occurs if and only if $f=3$. If $f=2$, we let $\mathcal{Q}_{1}=\left\{V_{1}, V_{2}\right\}$. If $f=3$ and $t=2$, we let $Y_{i}=X_{i}$ and we let $\mathcal{Q}_{1}=\left\{V_{1}, V_{2}\right\}$. If $f=3$ and $t>2$, we let $Y_{i}=X_{i} \backslash\left\{x_{i}^{1}, x_{i}^{2}, x_{i}^{3}\right\}$ and we let $\mathcal{Q}_{1}=\left\{V_{1}, V_{2}\right\}$.

If $f=4$, or $t=2$ and $f=5$, we let

$$
V_{2}=\bigcup_{i \in J_{3}} Y_{i} \cup \bigcup_{i \in J_{4}}\left\{x_{i}^{1}, x_{i}^{2}\right\}
$$

where $Y_{i}=\left\{x_{i}^{1}\right\}$ if $t=2$ and $Y_{i}=\emptyset$ otherwise. Next, if $f=5$ and $t>2$, we let

$$
V_{2}=\bigcup_{i \in J_{4}}\left\{x_{i}^{1}\right\} \cup \bigcup_{i \in J_{5}}\left\{x_{i}^{1}, x_{i}^{2}\right\} .
$$

Finally we let $V_{3}=X \backslash\left(V_{1} \cup V_{2}\right)$. The reader can easily verify that $\Sigma_{\mathcal{Q}_{1}}^{\mathcal{M}}$ stabilizes the partition $\mathcal{J}$ and hence acts trivially on $\mathcal{M}$, i.e. $\Sigma_{\mathcal{Q}_{1}} \subseteq \Sigma_{\mathcal{M}}$ and hence $\Sigma_{\sigma\left(\mathcal{Q}_{1}\right)} \subseteq \Sigma_{\mathcal{M}}$, for all $\sigma \in \Sigma$.

Lemma 7.1.2. Let $n \geq 2$ and let $\Sigma$ be a transitive solvable permutation group on a set $X$ of size $n$. Let $\mathcal{M}=\left\{X_{1}, \ldots, X_{m}\right\}(m \geq 1)$ be a partition of $X$ stabilized by $\Sigma$, such that $\left|X_{i}\right| \geq 2$ and such that $\Sigma^{X_{i}}$ is primitive. Let $f$ be a positive integer and for each $1 \leq i \leq m$, let $\mathcal{P}_{i}=\left\{X_{i}^{1}, \ldots, X_{i}^{f}\right\}$ be a partition of $X_{i}$. Define the partition $\mathcal{Q}_{2}=\left\{W_{1}, \ldots, W_{f}\right\}$ of $X$ by

$$
W_{t}=\bigcup_{j=1}^{m} X_{j}^{t}, \quad 1 \leq t \leq f .
$$

Then

(1) If $\Sigma^{X_{i}} ¥ \mathrm{~S}_{4}$, then there exist $2 \leq f \leq 3$ and partitions $\mathcal{P}_{i}, 1 \leq i \leq m$, so that the partition $\mathcal{Q}_{2}$ satisfies $\Sigma_{\mathcal{M}, \mathcal{Q}_{2}}=1$.

(2) If $\Sigma^{X_{i}} \cong \mathrm{S}_{4}$, then for $f=4$, the partitions $\mathcal{P}_{i}$ of $X_{i}$ to singletons, $1 \leq i \leq m$, yield a partition $\mathcal{Q}_{2}$ so that $\Sigma_{\mathcal{M}, \mathcal{Q}_{2}}=1$.

(3) If $\Sigma^{X_{i}} \cong \mathrm{S}_{4}$, then given subsets $Y_{i}^{1}, Y_{i}^{2} \subseteq X_{i}$, with $\left|Y_{i}^{j}\right|=2$ and $X_{i}=Y_{i}^{1} \cup Y_{i}^{2}$, $1 \leq i \leq m$, the partitions $\mathcal{P}_{i}:=\left\{Y_{i}^{1}, Y_{i}^{2}\right\}, 1 \leq i \leq m$ (so that $f=2$ and $X_{i}^{j}=Y_{i}^{j}, 1 \leq j \leq 2$ ), yield a partition $\mathcal{Q}_{2}$ such that $\Sigma_{\mathcal{M}, \mathcal{Q}_{2}}$ fixes $Y_{i}^{j}$ as a set, for all $1 \leq i \leq m$ and $1 \leq j \leq 2$.

Proof. In (1) take $\mathcal{P}_{i}$ so that $\Sigma_{\mathcal{P}_{i}}^{X_{i}}=1$. The existence of $\mathcal{P}_{i}$ in (1) is guaranteed by Corollary 2.2 in [25], which says that given a primitive solvable permutation group $H$ on a set $\Omega$, there exists a partition $\mathcal{P}$ of $\Omega$ to at most 3 parts so that $H_{\mathcal{P}}=1$. Parts (2) and (3) are obvious.

Lemma 7.1.3. Let $n \geq 2$ and let $\Sigma$ be a transitive solvable permutation group on a set $X$ of size $n$. Let $\mathcal{M}:=\left\{X_{1}, \ldots, X_{m}\right\}(m \geq 1)$ be a partition of $X$ stabilized by $\Sigma$, such that $\left|X_{i}\right| \geq 2$ and such that $\Sigma^{X_{i}}$ is primitive. Let $\mathcal{Q}_{1}=\left\{V_{1}, \ldots, V_{e}\right\}$, $2 \leq e \leq 3$, and $\mathcal{Q}_{2}=\left\{W_{1}, \ldots, W_{f}\right\}, 2 \leq f \leq 4$, be partitions of $X$ as in Lemma 7.1 .1 and Lemma 7.1 .2 respectively. Then

(1) If $\mathcal{Q}_{2}$ is as in part (1) or (2) of Lemma 17.1.2, then for all $\sigma \in \Sigma$, we have $\Sigma_{\sigma\left(\mathcal{Q}_{1}\right), \mathcal{Q}_{2}}=1$. 
(2) If $\mathcal{Q}_{2}$ is as in part (3) of Lemma 17.1.2, then for all $\sigma \in \Sigma$, we have $\Sigma_{\sigma\left(\mathcal{Q}_{1}\right), \mathcal{Q}_{2}} \subseteq$ $\Sigma_{\mathcal{M}}$, and for all $1 \leq i \leq m$, we have $\Sigma_{\sigma\left(\mathcal{Q}_{1}\right), \mathcal{Q}_{2}}^{X_{i}}$ is contained in the subgroup of $\operatorname{Sym}\left(X_{i}\right)$ fixing $Y_{i}^{j}$ as a set, for $j=1,2$, where $Y_{i}^{j}$ are as in part (3) of Lemma 7.1.2.

Proof. Let $\sigma \in \Sigma$. By the construction of $\mathcal{Q}_{1}$ we have $\Sigma_{\sigma\left(\mathcal{Q}_{1}\right), \mathcal{Q}_{2}} \subseteq \Sigma_{\mathcal{M}, \mathcal{Q}_{2}}$, so Lemma 7.1.2 completes the proof.

Notation 7.1.4. Let $\mathcal{P}=\left\{U_{1}, \ldots, U_{t}\right\}$ be a partition of $X$ and let $C_{1}, \ldots, C_{t} \subseteq L$ be distinct $\operatorname{Aut}(L)$ conjugacy classes. We let

$$
x\left(U_{1}, \ldots, U_{t}, C_{1}, \ldots, C_{t}\right)
$$

denote the set of elements $x$ of $K$ satisfying

$$
x_{j} \in C_{i}^{g_{j}} \text { if and only if } L_{j} \in U_{i}, \quad \text { for } 1 \leq j \leq n \text { and } 1 \leq i \leq t,
$$

where $K$ and $g_{1}, \ldots, g_{n}$ are as in Notation 7.3 .

Lemma 7.1.5. Let $\mathcal{Q}_{1}=\left\{V_{1}, \ldots, V_{e}\right\}$ be a partition of $X$ such that $\Sigma_{\mathcal{Q}_{1}} \leq \Sigma_{\mathcal{M}}$. Let $\mathcal{Q}_{2}=\left\{W_{1}, \ldots, W_{f}\right\}$ be a partition of $X$ as in Lemma 7.1.2. Let $B_{1}, \ldots, B_{e} \subseteq L$ be distinct $\operatorname{Aut}(L)$ classes and let $C_{1}, \ldots, C_{f} \subseteq L$ be distinct $\operatorname{Aut}(L)$ classes. Let $x \in x\left(V_{1}, \ldots, V_{e}, B_{1}, \ldots, B_{e}\right)$ and $y \in x\left(W_{1}, \ldots, W_{f}, C_{1}, \ldots, C_{f}\right)$. Then

(1) If $\mathcal{Q}_{2}$ is as in part (1) or (2) of Lemma 7.1.2, then $C_{G}\left(x^{g}, y\right)$ normalizes $L_{j}$, for all $g \in G$ and $1 \leq j \leq n$.

(2) Suppose $\mathcal{Q}_{2}$ is as in part (3) of Lemma 7.1 .2 , and set $Y_{i}^{j}=\left\{L_{i_{1}^{j}}, L_{i_{2}^{j}}\right\}, 1 \leq$ $i \leq m$ and $1 \leq j \leq 2$. Then $C_{G}\left(x^{g}, y\right)$ normalizes $L_{i_{1}^{j}} L_{i_{2}^{j}}, 1 \leq i \leq m$ and $1 \leq j \leq 2$, where $Y_{i}^{j}$ are as in part (3) of Lemma 7.1 .2

Proof. Let $g \in G$ and let $\sigma \in \Sigma$ be the image of $g$ in $\Sigma$. Note that by the choice of $x$, the image of $C_{G}\left(x^{g}\right)$ in $\Sigma$ fixes the partition $\sigma\left(\mathcal{Q}_{1}\right)$. Hence the image of $C_{G}\left(x^{g}\right)$ in $\Sigma$ acts trivially on $\mathcal{M}$ (of course $\mathcal{M}$ is as in (iii) of subsection 7.1). Let $\Theta$ be the image of $C_{G}\left(x^{g}, y\right)$ in $\Sigma$. Then, by the above, and by the choice of $y, \Theta \subseteq \Sigma_{\sigma\left(Q_{1}\right), Q_{2}}$, so the lemma holds by the choice of $\mathcal{Q}_{2}$, and by Lemma 7.1 .3

Lemma 7.1.6. Let $\mathcal{B}, \mathcal{C} \subseteq L$ be $\operatorname{Aut}(L)$ conjugacy classes. Consider the set of cyclic subgroups $\{\langle u\rangle \mid u \in \mathcal{B}\}$ and let $\mathcal{O}_{1}, \ldots, \mathcal{O}_{\mu}$ be the orbits of $L$ (via conjugation) on this set. Assume that for all $v \in \mathcal{C}$ and all $t \in \Delta_{\operatorname{Aut}(L)}(v)$ and every orbit $\mathcal{O} \in\left\{\mathcal{O}_{1}, \ldots, \mathcal{O}_{\mu}\right\}$, there exists $\left\langle u^{\prime}\right\rangle \in \mathcal{O}$ such that one of the following holds:

(a) $d_{\operatorname{Aut}(L)}\left(u^{\prime}, v\right) \geq 4$, or

(b) $d_{\mathrm{Aut}(L)}\left(u^{\prime}, v\right) \geq 3 \leq d_{\operatorname{Aut}(L)}\left(u^{\prime}, t\right)$, or

(c) $(\mathrm{c} 1) d_{\mathrm{Aut}(L)}\left(u^{\prime}, v\right) \geq 3$ and there exists a unique minimal path $\pi\left(u^{\prime}, t\right):=$ $u^{\prime}, s, t$ from $u^{\prime}$ to $t$ in $\Delta_{\mathrm{Aut}(L)}$ (unique up to replacing $s$ by a power of $s$ ).

(c2) For $s$ as in $(c 1)$, there exists $h^{\prime} \in L$ such that $d_{\operatorname{Aut}(L)}\left(\left(u^{\prime}\right)^{h^{\prime}}, v\right) \geq 3$ and $t$ does not commute with any nontrivial element of $\left\langle s^{h^{\prime}}\right\rangle$.

Then $(\operatorname{Aut}(L), u, v, L)$ satisfies Property $\left(3 \frac{1}{2}\right)$, for all $u \in \mathcal{B}$ and $v \in \mathcal{C}$.

Proof. First notice that hypothesis (a) implies hypothesis (b) (with the same $u^{\prime}$ ). Let $u \in \mathcal{B}$ and $v \in \mathcal{C}$ and let $a \in \Delta_{\operatorname{Aut}(L)}(u)$ and $b \in \Delta_{\operatorname{Aut}(L)}(v)$. Let $\mathcal{O}$ be the orbit of $\langle u\rangle$ under the action of $L$ (via conjugation). Suppose there exists $\left\langle u^{\prime}\right\rangle \in \mathcal{O}$ such that $d_{\operatorname{Aut}(L)}\left(u^{\prime}, v\right) \geq 3 \leq d_{\operatorname{Aut}(L)}\left(u^{\prime}, b\right)$. Let $h \in L$ such that $\left\langle u^{h}\right\rangle=\left\langle u^{\prime}\right\rangle$. If $\left[a^{h}, b\right]=1$, then since $\left[a^{h}, u^{\prime}\right]=1$, we get $d_{\mathrm{Aut}(L)}\left(u^{\prime}, b\right) \leq 2$, a contradiction. So $\left[a^{h}, b\right] \neq 1$ as required. 
Suppose there exists $\left\langle u^{\prime}\right\rangle \in \mathcal{O}$ as in hypothesis (c) (where in hypothesis (c) replace $t$ by $b$ ). Let $q \in L$, with $\left\langle u^{q}\right\rangle=\left\langle u^{\prime}\right\rangle$. Then $a^{q} \in\langle s\rangle$, otherwise, $\left[a^{q}, b\right] \neq 1$ and we are done. Let $h^{\prime}$ be as in (c2) and set $h=q h^{\prime}$. Then $d_{\text {Aut }(L)}\left(u^{h}, v\right) \geq 3$, but $\left[a^{h}, b\right] \neq 1$, as required.

Lemma 7.1.7. Let $x, y \in K$ such that $d_{G}(x, y)=3$. Assume that $x, a, b, y$ is a path in $\Delta_{G}$ such that there exists $j \in\{1, \ldots, n\}$ with $a, b \notin C_{G}\left(L_{j}\right)$. Assume further that $x_{j} \neq 1 \neq y_{j}$, that $d_{\operatorname{Aut}\left(L_{j}\right)}\left(x_{j}, y_{j}\right) \geq 3$, and that $\left[a^{q}, b\right]=1$, for all $q \in G$ such that $d_{G}\left(x^{q}, y\right) \geq 3$. Then $a, b \in N_{G}\left(L_{j}\right)$.

Proof. Let $q \in L_{j}$ such that $d_{G}\left(x^{q^{-1}}, y\right) \geq 3$. Then $\left[a, b^{q}\right]=1$. Assume that $L_{j}^{a} \neq L_{j}$. We have

$$
w^{a b^{q}}=\left(\left(w^{a}\right)^{q^{-1}}\right)^{b q}=w^{a b q}, \quad \text { for all } w \in L_{j} .
$$

CASE 1: $L_{j}^{b} \neq L_{j}$.

In this case we have

$$
w^{b^{q} a}=w^{q^{-1} b a}, \quad \text { for all } w \in L_{j} .
$$

If $L_{j}^{a b} \neq L_{j}$, then $w^{a b q}=w^{a b}$, so from (iv) and (豆) we get that

$$
w^{a b}=w^{q^{-1} a b}, \quad \text { for all } w \in L_{j},
$$

that is, $w=w^{q^{-1}}$, for all $w \in L_{j}$, which implies that $q$ is in the center of $L_{j}$. This is a contradiction since we can choose $q \neq 1$ (e.g. $q=x_{j}$ ) and $L_{j}$ is simple.

Hence we may assume that $L_{j}^{a b}=L_{j}$. From (iv) and (四) we get that $w^{a b q}=$ $w^{q^{-1} a b}$, for all $w \in L_{j}$. It follows that $(a b) q(a b)^{-1}=q^{-1}$. Hence $a b$ inverts every element $q \in L_{j}$ such that $d_{G}\left(x^{q^{-1}}, y\right) \geq 3$. Note however that for $q \in$ $\left\{x_{j}, y_{j}, y_{j}^{-1} x_{j}^{-1}\right\}$, we have $d_{G}\left(x^{q^{-1}}, y\right) \geq 3$; so since $a b$ inverts them, we conclude that $\left[x_{j}, y_{j}\right]=1$, contradicting $d_{\mathrm{Aut}\left(L_{j}\right)}\left(x_{j}, y_{j}\right) \geq 3$.

CASE 2: $L_{j}^{b}=L_{j}$.

Here $L_{j}^{a b} \neq L_{j}$. Let $w \in L_{j}$. By (iv), $w^{a b^{q}}=w^{a b}$. Hence we get that $w^{b a}=w^{b^{q} a}$ and it follows that $w^{b}=w^{b^{q}}$, for all $w \in L_{j}$, and hence $b=b^{q}$, for all $q$. In particular, $b$ centralizes $q=x_{j}$. Note that since $b$ normalizes $L_{j}$ and centralizes $y, b$ centralizes $y_{j}$. But then, thinking of $b$ as an element of $\operatorname{Aut}\left(L_{j}\right)$, we get that $1 \neq b \in C_{\operatorname{Aut}\left(L_{j}\right)}\left(x_{j}, y_{j}\right)$, contradicting the hypotheses of the lemma.

It follows that $a \in N_{G}\left(L_{j}\right)$. Notice now that our hypotheses are symmetric with respect to $x$ and $y$, so, by symmetry, $b \in N_{G}\left(L_{j}\right)$.

Proposition 7.1.8. Each one of the following hypotheses implies that $G$ has Prop$\operatorname{erty}\left(3 \frac{1}{2}\right)$ :

(a) There exist six distinct conjugacy classes $B, C_{1}, \ldots, C_{5}$ of $\operatorname{Aut}(L)$, contained in $L$, such that for every $u \in B$ and $v \in \bigcup_{i=1}^{5} C_{i}$, we have $d_{\operatorname{Aut}(L)}(u, v) \geq 3$.

(b) There exist conjugacy classes $B, C_{1}, \ldots, C_{5}$ of $\operatorname{Aut}(L)$, contained in $L$, such that $C_{1}, \ldots, C_{5}$ are distinct and such that for every $\mathcal{C} \in\left\{C_{1}, \ldots, C_{5}\right\}$ if we set $\mathcal{B}:=B$, then $(\operatorname{Aut}(L), u, v, L)$ has Property $\left(3 \frac{1}{2}\right)$, for all $u \in \mathcal{B}$ and $v \in \mathcal{C}$. 
(c) There exist distinct Aut $(L)$ conjugacy classes $B_{1}, B_{2}, B_{3} \subseteq L$ and distinct $\operatorname{Aut}(L)$ classes $C_{1}, \ldots, C_{f} \subseteq L$ such that,

(c1) If $\Sigma^{X_{i}} ¥ \mathrm{~S}_{4}, f=3$, while if $\Sigma^{X_{i}} \cong \mathrm{S}_{4}, f=4$.

(c2) For every $\mathcal{B} \in\left\{B_{1}, B_{2}, B_{3}\right\}$ and $\mathcal{C} \in\left\{C_{1}, \ldots, C_{f}\right\}$, we have that $(\operatorname{Aut}(L)$, $u, v, L)$ has Property $\left(3 \frac{1}{2}\right)$, for all $u \in \mathcal{B}$ and $v \in \mathcal{C}$.

Proof. We show that there are elements $x, y \in K$ such that $(G, x, y)$ satisfy Property $\left(3 \frac{1}{2}\right)$. Let $y$ be the following element. First we let $\mathcal{Q}_{2}:=\left\{W_{1}, \ldots, W_{f}\right\}$ be a partition of $X$ satisfying the following. In (a) and (b) we require that $2 \leq f \leq 5$ and that $\Sigma_{\mathcal{Q}_{2}}=1$. In (c) we require that $\Sigma_{\mathcal{M}, \mathcal{Q}_{2}}=1$. The existence of $\mathcal{Q}_{2}$ is guaranteed by Theorem 1.2 in [27] (see also Proposition 2.5 of [25]) in cases (a) and (b) and by Lemma 7.1.2(1) and 7.1.2(2), in case (c). We let

$$
y \in x\left(W_{1}, \ldots, W_{f}, C_{1}, \ldots, C_{f}\right\}
$$

(see Notation (7.1.4). Next we pick $x$ as follows. In the cases (a) and (b), we let $x \in K$ be an element such that $x_{i} \in B^{g_{i}}$, and $d_{\operatorname{Aut}(L)}\left(x_{i}, y_{i}\right) \geq 3$, for all $1 \leq i \leq n$. The existence of $x_{i}$ in (a) is guaranteed by the hypotheses and in (b) it is part of the definition of Property $\left(3 \frac{1}{2}\right)$. In case (c), we first pick a partition $\mathcal{Q}_{1}=\left\{V_{1}, \ldots, V_{e}\right\}$, $2 \leq e \leq 3$, of $X$ such that $\Sigma_{\mathcal{Q}_{1}} \subseteq \Sigma_{\mathcal{M}}$. The existence of $\mathcal{Q}_{1}$ is guaranteed by Lemma 7.1.1 Now that we have $\mathcal{Q}_{1}$, we let $x \in x\left(V_{1}, \ldots, V_{e}, B_{1}, \ldots, B_{e}\right)$ such that $d_{\mathrm{Aut}(L)}\left(x_{i}, y_{i}\right) \geq 3$ (again using the definition of Property $\left(3 \frac{1}{2}\right)$ ).

It is clear that in (a) and (b) the image of $C_{G}(y)$ in $\Sigma$ is contained in $\Sigma_{\mathcal{Q}_{2}}=1$, so $C_{G}(y)$ normalizes $L_{i}$, for all $1 \leq i \leq n$. Also in (c), by Lemma 7.1.5 (1), $C_{G}\left(x^{g}, y\right)$ normalizes $L_{i}, 1 \leq i \leq n$, for all $g \in G$. From the choice of $x_{i}, 1 \leq i \leq n$, we get that $d_{G}(x, y) \geq 3$.

We now show that $(G, x, y)$ satisfy Property $\left(3 \frac{1}{2}\right)$. Notice that given $g \in G$, in all three cases (a), (b) and (c), we can find $h \in K$, such that $d_{G}\left(x^{g h}, y\right) \geq 3$. This is because, as we indicated above, for any $h \in K, C_{G}\left(x^{g h}, y\right)$ normalizes $L_{i}$, for all $i$. Now our hypotheses allow us to find $h_{i} \in L_{i}$, such that $d_{\operatorname{Aut}\left(L_{i}\right)}\left(\left(x^{g}\right)_{i}^{h_{i}}, y_{i}\right) \geq 3$, and then for $h=h_{1} \cdots h_{n}$, we have $C_{G}\left(x^{g h}, y\right)=1$, that is, $d_{G}\left(x^{g h}, y\right) \geq 3$.

Assume that $(G, x, y)$ fail to satisfy Property $\left(3 \frac{1}{2}\right)$. Then there exists a path $x, a, b, y$ in $\Delta_{G}$ such that for all $g \in G$ with $d_{G}\left(x^{g}, y\right) \geq 3$, we have $\left[a^{g}, b\right]=1$. Let $j, k \in\{1, \ldots, n\}$ such that $a \notin C_{G}\left(L_{k}\right)$ and $b \notin C_{G}\left(L_{j}\right)$. Let $q \in G$ with $L_{k}^{q}=L_{j}$, and such that $d_{\operatorname{Aut}\left(L_{i}\right)}\left(\left(x^{q}\right)_{i}, y_{i}\right) \geq 3$, for all $1 \leq i \leq n$ (and hence $d_{G}\left(x^{q}, y\right) \geq 3$ ). The existence of $q$ is guaranteed since $\Sigma$ is transitive and by the previous paragraph of the proof. Then $x^{q}, a^{q}, b, y$ is a path in $\Delta_{G}$ and replacing $x$ by $x^{q}$ and $a$ by $a^{q}$ we may assume that $a, b \notin C_{G}\left(L_{j}\right)$. By Lemma 7.1.7, $a$ and $b$ normalize $L_{j}$. Notice now that for every $g \in L_{j}$ such that $d_{\operatorname{Aut}\left(L_{j}\right)}\left(x_{j}^{g}, y_{j}\right) \geq 3$, we have $d_{G}\left(x^{g}, y\right) \geq 3$, so $\left[a^{g}, b\right]=1$. Thinking of $a, b$ as elements of Aut $\left(L_{j}\right)$, we see that (Aut $\left.\left(L_{j}\right), x_{j}, y_{j}, L_{j}\right)$ do not satisfy Property $\left(3 \frac{1}{2}\right)$. This is a contradiction to hypothesis (b) and (c2), in the cases (b) and (c). It is a contradiction in case (a) as well, because in this case (Aut $\left.\left(L_{j}\right), x_{j}, y_{j}, L_{j}\right)$ has Property $\left(3 \frac{1}{2}\right)$ as well. To see this note that for any two conjugacy classes $\mathcal{B}$ and $\mathcal{C}$ of $L$ such that $d_{\operatorname{Aut}(L)}(u, v) \geq 3$, for all $u \in \mathcal{B}$ and $v \in \mathcal{C}$, $(\operatorname{Aut}(L), u, v, L)$ satisfy Property $\left(3 \frac{1}{2}\right)$, for any $u \in \mathcal{B}$ and $v \in \mathcal{C}$, because failure to do so violates the simplicity of $L$.

In our applications of part (b) of Proposition 7.1.8 we will use the following corollary. 
Corollary 7.1.9. Suppose that there are Aut $(L)$ conjugacy classes $B, C_{1}, \ldots, C_{5}$, contained in $L$, such that $C_{1}, \ldots, C_{5}$ are distinct and such that $L$ is transitive via conjugation on $\{\langle u\rangle \mid u \in B\}$. For $1 \neq w \in \operatorname{Aut}(L)$, let $\delta(w):=\mid\{u \in B \mid$ $\left.d_{\operatorname{Aut}(L)}(u, w) \leq 2\right\} \mid$. Assume that $\delta(w)<\frac{1}{2}|B|$, for all $1 \neq w \in \operatorname{Aut}(L)$, or, more generally, that $\delta(v)+\delta(w)<|B|$, for all $1 \neq w \in \operatorname{Aut}(L)$ and $v \in \bigcup_{i=1}^{5} C_{i}$. Then $G$ has Property $\left(3 \frac{1}{2}\right)$.

Proof. We show that $L$ satisfies hypothesis (b) of Proposition 7.1.8. For this we show that if we set $\mathcal{B}:=B$, then for all $\mathcal{C} \in\left\{C_{1}, \ldots, C_{5}\right\}$, the pair $\mathcal{B}, \mathcal{C}$ satisfies hypothesis (b) of Lemma 7.1.6, By our assumptions, given $v \in \bigcup_{i=1}^{5} C_{i}$ and $1 \neq$ $w \in \operatorname{Aut}(L)$, there exists $u^{\prime} \in B$ such that $d_{\operatorname{Aut}(L)}\left(u^{\prime}, v\right) \geq 3 \leq d_{\operatorname{Aut}(L)}\left(u^{\prime}, w\right)$. Now let $\mathcal{C} \in\left\{C_{1}, \ldots, C_{5}\right\}$ and pick $v \in \mathcal{C}$ and $t \in \Delta_{\text {Aut }(L)}(v)$. Then there exists $u^{\prime} \in B$ such that $d_{\operatorname{Aut}(L)}\left(u^{\prime}, v\right) \geq 3 \leq d_{\operatorname{Aut}(L)}\left(u^{\prime}, t\right)$. Hence $u^{\prime}$ is the element required in part (b) of Lemma 7.1.6.

The purpose of the next three lemmas is to handle the case when $L=\mathrm{A}_{5}$ in Theorem [7.2. Now the case when $L=\mathrm{A}_{5}$ and $\Sigma^{X_{i}} ¥ \mathrm{~S}_{4}$ can be handled using Proposition 7.1.8. So the next three lemmas actually deal with the case when $L=\mathrm{A}_{5}$ and $\Sigma^{X_{i}} \cong \mathrm{S}_{4}$; however we mention that in Lemma 7.1.13 we are not assuming $\Sigma^{X_{i}} \cong \mathrm{S}_{4}$. Though the lemmas are formulated for general $L$, the reader may think of $L=\mathrm{A}_{5}$. We assume that $C_{1}, C_{2}, C_{3} \subseteq L$ are three distinct $\operatorname{Aut}(L)$ conjugacy classes.

Notation 7.1.10. Suppose $\Sigma^{X_{i}} \cong \mathrm{S}_{4}$. We let $\mathcal{Q}_{1}=\left(V_{1}, \ldots, V_{e}\right)$ be as in Lemma7.1.1 and we let $\mathcal{Q}_{2}=\left(W_{1}, W_{2}\right)$ be as in part (3) of Lemma 7.1.2 the sets $Y_{i}^{j}=\left\{L_{i_{1}^{j}}, L_{i_{2}^{j}}\right\}$, $1 \leq i \leq m$ and $1 \leq j \leq 2$, are also as given in part (3) of Lemma 7.1.2. We let $x \in x\left(V_{1}, \ldots, V_{e}, C_{1}, \ldots, C_{e}\right)$ and $y \in x\left(W_{1}, W_{2}, C_{1}, C_{2}\right)$.

Lemma 7.1.11. The following two assertions hold:

(1) Let $k, l \in\{1, \ldots, n\}$ be two distinct indices. Let $x_{k} x_{l}, y_{k} y_{l} \in L_{k} L_{l}$ such that $C_{\mathrm{Aut}\left(L_{k}\right)}\left(x_{k}, y_{k}\right)=1$. Then there exists at most one orbit $\mathcal{O}_{l} \subseteq x_{l}^{\operatorname{Aut}\left(L_{l}\right)} \cap$ $\Delta_{\mathrm{Aut}\left(L_{l}\right)}^{\geq 3}\left(y_{l}\right)$ under the action of $C_{\mathrm{Aut}\left(L_{l}\right)}\left(y_{l}\right)$ (acting by conjugation), such that for $x_{l}^{\prime} \in \mathcal{O}_{l}$, we have $C_{\mathrm{Aut}\left(L_{k} L_{l}\right)}\left(x_{k} x_{l}^{\prime}, y_{k} y_{l}\right) \neq 1$.

(2) Assume that $\Sigma^{X_{i}} \cong \mathrm{S}_{4}$ and let $x, y$ be as in Notation 7.1.10. Assume further that $d_{\operatorname{Aut}\left(L_{i}\right)}\left(x_{i}, y_{i}\right) \geq 3$, for all $1 \leq i \leq n$, and that $d_{G}(x, y) \geq 3$. Then for each $1 \leq l \leq n$, if the set

$$
\left\{x_{l}^{h} \in x_{l}^{L_{l}} \cap \Delta_{\mathrm{Aut}\left(L_{l}\right)}^{\geq 3}\left(y_{l}\right) \mid h \in L_{l} \text { and } d_{G}\left(x^{h}, y\right) \leq 2\right\}
$$

is not empty, then it is contained in an orbit $\mathcal{O}_{l} \subseteq x_{l}^{\operatorname{Aut}\left(L_{l}\right)} \cap \Delta_{\operatorname{Aut}\left(L_{l}\right)}^{\geq 3}\left(y_{l}\right)$, under the action of $C_{\mathrm{Aut}\left(L_{l}\right)}\left(y_{l}\right)$.

Proof. (1) Suppose that $x_{l}^{\prime}, x_{l}^{\prime \prime} \in x_{l}^{\operatorname{Aut}\left(L_{l}\right)} \cap \Delta_{\operatorname{Aut}\left(L_{l}\right)}^{\geq 3}\left(y_{l}\right)$ are such that there exist $1 \neq$ $q \in C_{\operatorname{Aut}\left(L_{k} L_{l}\right)}\left(x_{k} x_{l}^{\prime}, y_{k} y_{l}\right)$ and $1 \neq s \in C_{\operatorname{Aut}\left(L_{k} L_{l}\right)}\left(x_{k} x_{l}^{\prime \prime}, y_{k} y_{l}\right)$. Now $q^{2}$ normalizes each $L_{j}, j=k, l$, so since $C_{\mathrm{Aut}\left(L_{k}\right)}\left(x_{k}, y_{k}\right)=1=C_{\mathrm{Aut}\left(L_{l}\right)}\left(x_{l}^{\prime}, y_{l}\right), q^{2}=1$, and $q$ interchanges $L_{k}$ and $L_{l}$. Thus $x_{k}^{q}=x_{l}^{\prime}, y_{k}^{q}=y_{l}$.

Next, as above we must have $x_{k}^{s}=x_{l}^{\prime \prime}, y_{k}^{s}=y_{l}$, and $s^{2}=1$, so $s=u v q$, with $u \in C_{\mathrm{Aut}\left(L_{k}\right)}\left(y_{k}\right)$ and $v \in C_{\mathrm{Aut}\left(L_{l}\right)}\left(y_{l}\right)$. Then

$$
x_{l}^{\prime \prime}=x_{k}^{s}=\left(x_{k}^{q}\right)^{q^{-1} u v q}=\left(x_{l}^{\prime}\right)^{\left(q^{-1} u v q\right)}=\left(x_{l}^{\prime}\right)^{q^{-1} u q},
$$


but $q^{-1} u q \in C_{\operatorname{Aut}\left(L_{l}\right)}\left(y_{l}\right)$, so we see that $x_{l}^{\prime}$ and $x_{l}^{\prime \prime}$ are in the same orbit of $x_{l}^{\operatorname{Aut}(L)} \cap$ $\Delta_{\text {Aut }\left(L_{l}\right)}^{\geq 3}\left(y_{l}\right)$ under the action of $C_{\operatorname{Aut}\left(L_{l}\right)}\left(y_{l}\right)$.

(2) Let $l \in\{1, \ldots, n\}$. Without loss we may assume that $L_{l} \in Y_{1}^{1}$ and we will denote $\left\{L_{k}\right\}=Y_{1}^{1} \backslash\left\{L_{l}\right\}$. Let $h, h^{\prime} \in L_{l}$ such that $x_{l}^{h}, x_{l}^{h^{\prime}} \in \Delta_{\operatorname{Aut}\left(L_{l}\right)}^{\geq 3}\left(y_{l}\right)$, and suppose that $d_{G}\left(x^{h}, y\right) \leq 2 \geq d_{G}\left(x^{h^{\prime}}, y\right)$. Let $1 \neq q \in C_{G}\left(x^{h}, y\right)$ and $s \in C_{G}\left(x^{h^{\prime}}, y\right)$. Notice that by the choice of $x, y$ and by Lemma 7.1.5 $q$ normalizes $L_{k} L_{l}$, and if $q \in C_{G}\left(L_{k} L_{l}\right)$, then $q \in C_{G}(x, y)$, contradicting $d_{G}(x, y) \geq 3$. Similarly, $s$ restricts to a nontrivial automorphism of $L_{k} L_{l}$. Hence, thinking of $q$ and $s$ as elements of $\operatorname{Aut}\left(L_{k} L_{l}\right)$, we see that $C_{\operatorname{Aut}\left(L_{k} L_{l}\right)}\left(x_{k} x_{l}^{h}, y_{k} y_{l}\right) \neq 1 \neq C_{\operatorname{Aut}\left(L_{k} L_{l}\right)}\left(x_{k} x_{l}^{h^{\prime}}, y_{k} y_{l}\right)$. It follows from part (1) that $x_{l}^{h}, x_{l}^{h^{\prime}}$ are in the same orbit under the action of $C_{\mathrm{Aut}\left(L_{l}\right)}\left(y_{l}\right)$.

Lemma 7.1.12. Assume $\Sigma^{X_{i}} \cong \mathrm{S}_{4}$ and let $x, y \in K$ be as in Notation 7.1.10. Suppose that for all $v \in C_{1} \cup C_{2}$ and $1 \leq j \leq 3$ and each $u \in C_{j}$, we have that $u^{L} \cap \Delta_{\operatorname{Aut}(L)}^{\geq 3}(v)$ is nonempty and is not contained in an orbit of $C_{\operatorname{Aut}(L)}(v)$ on $\Delta_{\operatorname{Aut}(L)}^{\geq 3}(v)$ (acting via conjugation). Then for all $g \in G$, there exists $h \in K$ such that $d_{G}\left(x^{g h}, y\right) \geq 3$ and such that $d_{\mathrm{Aut}\left(L_{i}\right)}\left(\left(x^{g h}\right)_{i}, y_{i}\right) \geq 3$, for all $1 \leq i \leq n$.

Proof. Let $g \in G$ and set $z:=x^{g}$. By Lemma 7.1.5, $C_{G}(z, y)$ normalizes $L_{i_{1}^{j}} L_{i_{2}^{j}}$, $1 \leq i \leq m$ and $1 \leq j \leq 2$. For each $L_{i} \in\left\{L_{1}, \ldots, L_{n}\right\}$, pick $r_{i} \in L_{i}$ so that $z_{i}^{r_{i}} \in \Delta_{\text {Aut }\left(L_{i}\right)}^{\geq 3}\left(y_{i}\right)$. This can be done by hypothesis. Replacing $z$ by $z^{r}$ (where $\left.r=r_{1} \cdots r_{n}\right)$, we may assume that $d_{\text {Aut }\left(L_{i}\right)}\left(z_{i}, y_{i}\right) \geq 3$, for all $1 \leq i \leq n$. Let $1 \leq i \leq m$ and $1 \leq j \leq 2$ and set $k=i_{1}^{j}, l=i_{2}^{j}$. Set $h_{k}=1$ and pick $h_{l} \in L_{l}$, so that $d_{\operatorname{Aut}(L)}\left(z_{l}^{h_{l}}, y_{l}\right) \geq 3$ and $\left.C_{\operatorname{Aut}\left(L_{k} L_{l}\right.}\right)\left(z_{k} z_{l}^{h_{l}}, y_{k} y_{l}\right)=1$. This can be done using the hypotheses of the lemma and using Lemma 7.1.11(1). By construction $h=h_{1} \cdots h_{n}$ satisfies $d_{G}\left(x^{g h}, y\right) \geq 3$ and $d_{\text {Aut }\left(L_{i}\right)}\left(\left(x^{g h}\right)_{i}, y_{i}\right) \geq 3$, for all $1 \leq i \leq n$, as required.

Lemma 7.1.13 below is our tool for showing that when $L \cong \mathrm{A}_{5}$ and $\Sigma^{X_{i}} \cong \mathrm{S}_{4}, G$ satisfies Property $\left(3 \frac{1}{2}\right)$. Since this lemma is quite technical, a word of explanation is needed here. Suppose $\Sigma^{X_{i}} \cong \mathrm{S}_{4}$ and that we found elements $x, y$ as in Notation 7.1.10 such that $d_{G}(x, y) \geq 3$ and such that $d_{\text {Aut }\left(L_{i}\right)}\left(x_{i}, y_{i}\right) \geq 3$, for all $i$. We wish to show that $(G, x, y)$ satisfy Property $\left(3 \frac{1}{2}\right)$. For this we need enough elements $h \in L_{j}$ (some $j$ ), such that $d_{G}\left(x^{h}, y\right) \geq 3$. Thus we consider the set $x_{j}^{L_{j}} \cap \Delta_{\text {Aut }\left(L_{j}\right)}^{\geq 3}\left(y_{j}\right)$ and we want to pick $h \in L_{j}$ such that $x_{j}^{h}$ is in this set. The fact that $d_{\operatorname{Aut}(L)}\left(x_{j}^{h}, y_{j}\right) \geq 3$ "almost" guarantees $d_{G}\left(x^{h}, y\right) \geq 3$, except perhaps when $x_{j}^{h}$ belongs to the "bad" orbit of Lemma 7.1.11 2 ) (with respect to the action of $C_{\mathrm{Aut}\left(L_{j}\right)}\left(y_{j}\right)$ via conjugation). Since we don't know which is the "bad" orbit, we must make sure that: (1) there is more than one orbit, and (2) that for each orbit of $C_{\operatorname{Aut}\left(L_{j}\right)}\left(y_{j}\right)$ on the set $x_{j}^{\operatorname{Aut}\left(L_{j}\right)} \cap \Delta_{\operatorname{Aut}\left(L_{j}\right)}^{\geq 3}\left(y_{j}\right)$, there is an element $x_{j}^{h} \in x_{j}^{L_{j}} \cap \Delta_{\operatorname{Aut}\left(L_{j}\right)}^{\geq 3}\left(y_{j}\right)$ but not in this orbit and such that furthermore $h \in L_{j}$ has some additional "nice" properties. This is the content of hypothesis (b) of Lemma 7.1.13.

Lemma 7.1.13. Assume that for each $v \in C_{1} \cup C_{2}$ we have

(a) For all $1 \leq j \leq 3, C_{\operatorname{Aut}(L)}(v)$ has more than one orbit on $C_{j} \cap \Delta_{\operatorname{Aut}(L)}^{\geq 3}(v)$ (acting via conjugation). 
(b) For each orbit $\mathcal{O}$ of $C_{\mathrm{Aut}(L)}(v)$ on $C_{j} \cap \Delta_{\mathrm{Aut}(L)}^{\geq 3}(v)$, each $u \in C_{j}, s \in \Delta_{\mathrm{Aut}(L)}(u)$ and $t \in \Delta_{\operatorname{Aut}(L)}(v)$, there exists $h \in L$ such that $u^{h} \in \Delta_{\operatorname{Aut}(L)}^{\geq 3}(v) \backslash \mathcal{O}$ and $\left[s^{h}, t\right] \neq 1$.

Assume further that

(c) For every $\mathcal{B}, \mathcal{C} \in\left\{C_{1}, C_{2}, C_{3}\right\}$, we have that (Aut $\left.(L), u, v, L\right)$ has Property $\left(3 \frac{1}{2}\right)$, for all $u \in \mathcal{B}$ and $v \in \mathcal{C}$.

Then $G$ satisfies Property $\left(3 \frac{1}{2}\right)$.

Proof. If $\Sigma^{X_{i}} ¥ \mathrm{~S}_{4}$, then by hypothesis (c) the hypotheses of part (c) of Proposition 7.1 .8 hold, so by that proposition, we are done.

Hence we may assume that $\Sigma^{X_{i}} \cong \mathrm{S}_{4}$. Let $x, y$ be as in Notation [7.1.10, Notice that our hypotheses (a) and (b) imply the hypotheses of Lemma 7.1.12, hence replacing $x$ by a conjugate of $x$, we may (and we do) assume that $d_{G}(x, y) \geq 3$ and $d_{\mathrm{Aut}\left(L_{i}\right)}\left(x_{i}, y_{i}\right) \geq 3$, for all $1 \leq i \leq n$. Assume that $(G, x, y)$ fail to satisfy Property $\left(3 \frac{1}{2}\right)$. Then there exists a path $x, a, b, y$ in $\Delta_{G}$ such that for all $g \in G$ with $d_{G}\left(x^{g}, y\right) \geq 3$, we have $\left[a^{g}, b\right]=1$. By the transitivity of $\Sigma$ and using Lemma 7.1.12, we may replace $x$ by a conjugate of $x$, so that we may assume that there exists $j \in\{1, \ldots, n\}$ such that $a, b \notin C_{G}\left(L_{j}\right)$. By Lemma 7.1.7 $a, b \in N_{G}\left(L_{j}\right)$. Now identify $L_{j}$ with $L$ (via conjugation by $g_{j}^{-1}$ ). Let $k \in\{1,2,3\}$ be such that $x_{j} \in C_{k}$. Let $\mathcal{O}$ be an orbit of $C_{\operatorname{Aut}\left(L_{j}\right)}\left(y_{j}\right)$ on $C_{k} \cap \Delta_{\operatorname{Aut}\left(L_{j}\right)}^{\geq 3}\left(y_{j}\right)$ such that for $h \in L_{j}$ with $x_{j}^{h} \in \Delta_{\text {Aut }\left(L_{j}\right)}^{\geq 3}\left(y_{j}\right) \backslash \mathcal{O}$, we have $d_{G}\left(x^{h}, y\right) \geq 3$ (we take $\mathcal{O}$ to be the "bad" orbit of Lemma 7.1.11(2) if it exists and otherwise $\mathcal{O}$ is any orbit). Note now that by hypothesis (b), there exists $q \in L_{j}$ such that $x_{j}^{q} \in \Delta_{\operatorname{Aut}\left(L_{j}\right)}^{\geq 3}\left(y_{j}\right) \backslash \mathcal{O}$ and $\left[a^{q}, b\right] \neq 1$. But then also $d_{G}\left(x^{q}, y\right) \geq 3$ and this supplies a contradiction to the choice of the path $x, a, b, y$.

7.2. The generic case. In this subsection $L$ is a finite nonabelian simple group such that $L$ is not isomorphic to one of the groups $\operatorname{PSL}(2, q), q=5,7,8,9,11,16,27$, $\operatorname{PSL}(3,4)$ or $\mathrm{PSO}^{+}(8,2)$.

We consider the following condition:

(Gen) There exist six distinct nonidentity conjugacy classes $B, C_{1}, \ldots, C_{5}$ of $\operatorname{Aut}(L)$ contained in $L$, such that $d_{\mathrm{Aut}(L)}(r, s) \geq 3$, for all $r \in B$ and $s \in \bigcup_{i=1}^{5} C_{i}$.

The purpose of this subsection is to prove Theorem 7.5, i.e. to prove

Theorem 7.2.1. L satisfies (Gen).

We start with the Alternating groups.

Lemma 7.2.2. Let $L \cong \mathrm{A}_{n}$. Then

(1) If $n \geq 7$ and $n$ is odd, then $L$ satisfies (Gen).

(2) If $n \geq 8$ and $n$ is even, then $L$ satisfies (Gen).

Proof. (1) Let $B$ be the $\operatorname{Aut}(L)$ conjugacy class of $n$ cycles. Note that if $C \subseteq L$ is an $\operatorname{Aut}(L)$ conjugacy class of elements of $L$ having precisely one fixed point, or having precisely two fixed points, then $d_{\operatorname{Aut}(L)}(b, c) \geq 3$, for all $b \in B$ and $c \in C$. It is clear that when $n \geq 9$, there are at least five distinct classes $C$ as above. If $n=7$, then for all conjugacy classes $C \neq B, b \in B$ and $c \in C$, one has $d_{\text {Aut }(L)}(b, c)=\infty$, so clearly $(G e n)$ holds in this case as well. 
(2) Let $B$ be the conjugacy class of $n-1$ cycles. Note that if $C \subseteq L$ is an $\operatorname{Aut}(L)$ conjugacy class of elements of $L$ having no fixed points, or having precisely two fixed points, then $d_{\operatorname{Aut}(L)}(b, c) \geq 3$, for $b \in B$ and $c \in C$. It is easily checked that for $n \geq 8$ there are at least five distinct classes $C$ as above.

For the Sporadic groups we have

Lemma 7.2.3. Assume $L$ is a Sporadic group not isomorphic to $J_{2}$ or $M c L$. Then (Gen) holds for L.

Proof. It is easy to check (using the ATLAS) that $L$ contains an $\operatorname{Aut}(L)$ conjugacy class $B$ such that $\langle b\rangle \backslash\{1\}$ is a connected component of $\Delta_{\operatorname{Aut}(L)}$, for each $b \in$ $B$. Since the number of $\operatorname{Aut}(L)$ conjugacy classes contained in $L$ is $\geq 6$, we are done.

Lemma 7.2.4. Assume $L \cong J_{2}$. Then $L$ satisfies $(G e n)$.

Proof. Let $B \subseteq L$ be the class of elements of order 7. Then for $b \in B$ we have $C_{\text {Aut }(L)}(b)=\langle b\rangle\langle t\rangle$, where $t$ is an (outer) involution with $C_{L}(t) \cong S L(3,2): 2$. It follows that if $C_{1}, \ldots, C_{5}$ are the $\operatorname{Aut}(L)$ conjugacy classes of elements of order $5,5,10,10,12$, then $B, C_{1}, \ldots, C_{5}$ satisfy $(G e n)$.

Lemma 7.2.5. Assume $L \cong M c L$. Then $L$ satisfies (Gen).

Proof. Let $B$ be the class of elements of order 11 . Then for $b \in B$, we have $C_{\mathrm{Aut}(L)}(b)=\langle b\rangle\langle t\rangle$, where $t$ is an (outer) involution with $C_{L}(t) \cong \mathrm{M}_{11}$. It follows that if $C_{1}, \ldots, C_{5}$ are $\operatorname{Aut}(L)$ conjugacy classes of elements of order $7,9,10,12,14$, then $B, C_{1}, \ldots, C_{5}$ satisfy $(G e n)$.

\section{The groups of Lie type}

The remainder of this subsection is devoted to the groups of Lie type. Let $L=L(q)$ be a simple group of Lie type defined over a field of order $q=p^{a}, p$ a prime. Let $G$ be the corresponding algebraic group, so that $L=\left(G_{\sigma}\right)^{\prime}$ for $\sigma$ a Frobenius morphism of $G$. Until the end of subsection 7.2 we let $A=\operatorname{Aut}(L)$. We will exhibit elements $x, y_{1}, \ldots, y_{5} \in L$ such that $x^{A}, y_{1}^{A}, \ldots, y_{5}^{A}$ are six distinct Aut $(L)$ conjugacy classes satisfying condition $(G e n)$.

We use the following notation for certain groups of Lie type. For $\epsilon= \pm 1$ we let $A_{n}^{\epsilon}, D_{n}^{\epsilon}, E_{6}^{\epsilon}$ be groups of Lie type as follows. If $\epsilon=1$, these are just the (untwisted) Chevalley groups $A_{n}(q), D_{n}(q), E_{6}(q)$, respectively; while if $\epsilon=-1$, we have the twisted groups ${ }^{2} A_{n}(q),{ }^{2} D_{n}(q),{ }^{2} E_{6}(q)$.

Elements of $A$ can be expressed as a product of inner, diagonal, field, and graph automorphisms (see Thms. 30 and 36 of [29]). It is also known that automorphisms of $L$ extend to morphisms of $G$ commuting with $\sigma$. Now $G_{\sigma}$ is the group of inner and diagonal automorphisms of $L$, so that $A / G_{\sigma}$ is generated by images of field and graph automorphisms of $G$ restricted to $L$. For example, if $\sigma$ is a field morphism of $G$ corresponding to $q=p^{a}$, there is a field morphism $\mu$ corresponding to $p$ such that $\mu^{a}=\sigma$ and $\mu$ acts on $L$ generating the group of field automorphisms.

Throughout this subsection, the term "graph automorphism" of $G$ will be used somewhat loosely. Let $\tau$ be a standard graph automorphism of $G$ (see p.156 of [29]). We will refer to any element in the $\operatorname{coset} G \tau$ of order equal to that of $\tau$ as a graph automorphism. Typically there exist just one or two $G$-classes of such elements. 
For instance in $E_{6}$ there are two classes of such morphisms. They have fixed points $F_{4}$ and $C_{4}$, except for $p=2$, where the fixed points are $F_{4}$ and the centralizer in $F_{4}$ of a long root element of $E_{6}$ contained in $F_{4}$.

When $L=P S p(4, q), F_{4}(q)$ or $G_{2}(q)$ with $p=2,2,3$ respectively, there is an endomorphism $\delta$ of the algebraic group $G$ that commutes with $\sigma$ and interchanges the root group corresponding to long and short roots. Also $\delta^{2}$ generates the group of field morphisms. Here $A=L\left\langle\delta_{L}\right\rangle$, where $\delta_{L}$ is the restriction of $\delta$ to $L$. We will call $\delta_{L}$ a "special graph automorphism". When $q=p^{a}$, with $a$ odd, the involution $\tau=\left(\delta_{L}\right)^{a}$ has fixed point group ${ }^{2} B_{2}(q),{ }^{2} F_{4}(q)$ or ${ }^{2} G_{2}(q)$, respectively, and $\tau$ is called an "involutory special graph automorphism".

When there is no danger of confusion we will sometimes identify an automorphism of $L$ with its extension to $G$.

Lemma 7.2.6. Let $u \in L$ be a regular unipotent element. Then $C_{A}(u)=U\langle\delta\rangle J$, where $U<G_{\sigma}$ is a unipotent group, $\delta$ generates the group of field automorphisms of $L$ and $J$ is a group of graph (or special graph) automorphisms.

Proof. As $u$ is regular we have $C_{G}(u)$ a unipotent group. Hence $C_{G_{\sigma}}(u)$ is a unipotent group. On the other hand, any unipotent element that is the product of root elements from positive root groups with nontrivial contribution for each fundamental root is regular and all regular unipotent elements are conjugate in $G$ (see III, 1.8 of [28]). It follows from the action of standard field automorphisms that each of these fix a regular unipotent element. The assertion follows. We make the additional remark that regular unipotent elements of $G$ are typically fixed by a graph automorphism. The one exception is $G=P S L_{n}(q)$ with $n$ odd and $q$ even.

Lemma 7.2.7. Let $a \in A \backslash G_{\sigma}$ have prime power order $r^{e}$ with $a^{r} \in G_{\sigma}$. Then a is the restriction to $L$ of a morphism $\delta$ of $G$ commuting with $\sigma$. Moreover, one of the following holds:

(i) There does not exist a graph or involutory special graph automorphism $\tau$ such that $a \in G_{\sigma} \tau$. In this case $G_{\delta}=G\left(q_{o}\right)$ for $q_{o}<q$.

(ii) $a \in G_{\sigma} \tau$ for some graph automorphism $\tau$ of $G$. In this case $C_{L}(a)$ is contained in $D_{\sigma}=D(q)$, where $D^{\sigma}=D<G$ is either a parabolic subgroup (only if $p=|\tau|)$ or a reductive group of semisimple rank strictly less than that of $G$.

(iii) $a \in G_{\sigma} \tau$, where $\tau$ is an involutory special graph automorphism of $P S p(4, q)$, $F_{4}(q)$ or $G_{2}(q)$. If $|a|=2$, then $C_{L}(a)={ }^{2} B_{2}(q),{ }^{2} F_{4}(q)$ or ${ }^{2} G_{2}(q)$, respectively. Otherwise, $C_{L}(a)$ is contained in a proper parabolic subgroup of PSp $(4, q)$ or $F_{4}(q)$ or a subgroup $S L_{2}(q) \cdot S L_{2}(q)$ of $G_{2}(q)$, respectively.

Proof. Fix $a \in A \backslash G_{\sigma}$ and recall that $a$ is the restriction to $L$ of a morphism commuting with $\sigma$. There is an element $g \in G_{\sigma}$ such that $a$ is induced by $g \cdot \mu$ for $\mu$ a field, graph-field, graph, or special graph automorphism of $G$ commuting with $\sigma$.

First suppose $\mu \notin G \tau$ for $\tau$ a graph or involutory special graph automorphism of $G$. Regarding $g \cdot \mu$ as a member of the coset $G \mu \subseteq \operatorname{Aut}(G)$ (automorphisms as abstract group), we see that $\mu$ involves a field morphism of $G$ corresponding to a prime power $q_{o}<q$. It then follows from Lang's theorem (see I, 2.2 of [28]) that $g \cdot \mu$ is $G$-conjugate to $\mu$. Since $G_{\mu}=G\left(q_{o}\right)$ for $q_{o}$ a proper divisor of $q$, part (i) follows.

Now suppose $a \in G_{\sigma} \tau$ for some graph automorphism $\tau$ of $G$ commuting with $\sigma$. We have $C_{L}(a) \leq C_{G}(a)_{\sigma}$ and $|\tau|=r$. So $r=2$ except possibly for $G=D_{4}$ where 
$r=3$. If $|a|=|\tau|$, then $a$ is a graph automorphism of $G$ and the centralizers of such automorphisms are known (for example see 1.1 of [11]). It follows that either $C_{G}(a)$ is reductive and we obtain the result by setting $D=C_{G}(a)$, or else $p=r$ and $C_{G}(a)$ has a nontrivial unipotent radical, and so by the Borel-Tits theorem it is contained in a canonical parabolic subgroup $D$ of $G$. Furthermore, since $C_{G}(a)$ is $\sigma$-invariant, the unipotent radical of $C_{G}(a)$ is $\sigma$-invariant. Consequently, $D$ can be chosen to be $\sigma$-invariant.

Now assume $|a|>|\tau|$ and let $t=a^{r}$. If $p \neq r$, then $t$ is contained in a maximal torus of $G$, so $C_{G}(t)$ is a subsystem subgroup and $a$ induces an involutory (or order 3 ) semisimple automorphism of this group (it cannot centralize $C_{G}(t)$ as maximal tori are selfcentralizing). The result follows with $D=C_{G}(a)$. Otherwise, $C_{G}(a)$ is contained in a $\sigma$-invariant canonical parabolic $D$, completing the proof of (ii).

Finally assume we are in the situation of (iii), so that $|a|=2^{e}$. If $a$ is an involution, then it is well known that $C_{L}(a)={ }^{2} B_{2}(q),{ }^{2} F_{4}(q)$ or ${ }^{2} G_{2}(q)$. Otherwise, $1 \neq a^{2} \in L$ so $C_{L}(a)$ centralizes an involution in $L$ and the assertion follows from well-known information on involution centralizers in these groups.

\section{The exceptional groups}

Let $L$ be a finite exceptional group.

Proposition 7.2.8. Condition (Gen) holds if $L=E_{8}(q), E_{7}(q), E_{6}^{\epsilon}(q), F_{4}(q)$.

Proof. In nearly all cases we take $x$ to be a regular unipotent element of $L$, so Lemma 7.2.6 implies $C_{A}(x)=U\langle\delta, \tau\rangle$, where $\delta$ generates the group of field automorphisms and $\tau$ is the identity or a graph automorphism. We will first choose $y_{1}, y_{2}$ as certain semisimple elements. The remaining elements $y_{3}, y_{4}, y_{5}$ will usually be taken as nonconjugate generators of $\left\langle y_{1}\right\rangle$ or $\left\langle y_{2}\right\rangle$. Recall that we aim at showing that for $A=\operatorname{Aut}(L)$, the classes $B=x^{A}$ and $C_{i}=y_{i}^{A}, 1 \leq i \leq 5$, satisfy condition $(G e n)$. In the following table we provide information on the order of the elements $y_{1}, y_{2}$ :

\begin{tabular}{ccc}
$G$ & $y_{1}$ & $y_{2}$ \\
\hline$E_{8}(q)$ & $\left(q^{9}-1\right) /(q-1)(3, q-1)$ & $\left(q^{9}+1\right) /(q+1)(3, q+1)$ \\
$E_{7}(q)$ & $\left(q^{8}-1\right) /(q-1)(4, q-1)$ & $\left(q^{7}+1\right) /(4, q+1)$ \\
$E_{6}^{\epsilon}(q)$ & $\left(q^{9}-\epsilon\right) /\left(q^{3}-\epsilon\right)(3, q-\epsilon)$ & $\left(q^{5}-\epsilon\right)(q+1) /(q-\epsilon)$ \\
$F_{4}(q)$ & $\left(q^{4}+1\right) /(2, q-1)$ & $\left(q^{4}-1\right) /(4, q-1)$ \\
\hline
\end{tabular}

The existence of the elements indicated follows immediately from the following containments, which in turn follow via Lang's theorem from the existence of standard subsystems of the root system. The elements are also described on p.34 of [9].

$E_{8}(q) \geq A_{8}^{\epsilon}$ (an image of $S L(9, q), S U(9, q)$ with kernel of order $\left.(3, q-\epsilon)\right)$.

$E_{7}(q) \geq A_{7}^{\epsilon}(q)$ (an image of $S L(8, q), S U(8, q)$ with kernel of order $(4, q-\epsilon)$ ).

$E_{6}^{\epsilon}(q)>A_{2}^{\epsilon}\left(q^{3}\right)$ and $A_{5}^{\epsilon}(q) A_{1}(q)$ (central product, no central 3-element).

$F_{4}(q)>B_{4}(q)>D_{4}^{\epsilon}(q)$ (simply connected).

(In the last case $y_{1}$ is the preimage in the spin group of an irreducible element of $S O^{-}(8, q)^{\prime}$, while $y_{2} \in S L(4, q) \times Z_{q-1}$ is the product of a Singer cycle of the first factor and a generator of the second factor.)

The proof now proceeds in a series of lemmas. 
Lemma 7.2.9. Let $y_{i} \in L$ as above. Then either $C_{G}\left(y_{i}\right)$ is a maximal torus, $T$, of $G$, or $L=F_{4}(2)$ and $i=2$.

Proof. We have $y_{i} \in T$ for a maximal torus $T<G$, so it will suffice to show that $C_{G}\left(y_{i}\right)$ is connected and has dimension equal to that of $T$. We first consider the dimension of the centralizer. Now $\operatorname{dim} C_{G}\left(y_{i}\right)=\operatorname{dim} C_{L(G)}\left(y_{i}\right)($ see p.28 of [8]), so it suffices to show that $\operatorname{dim} C_{L(G)}\left(y_{i}\right)=\operatorname{dim}(T)$.

We determine the action of $y_{i}$ on $L(G)$ by first computing the restriction of $L(G)$ to the subgroups indicated above. We do this at the level of algebraic groups, where the information is given explicitly in 2.1 and 8.4 of [12. The results are as follows:

$L\left(E_{8}\right) \downarrow A_{8}=L\left(A_{8}\right) \oplus V_{A_{8}}\left(\lambda_{3}\right) \oplus V_{A_{8}}\left(\lambda_{6}\right)$.

$L\left(E_{7}\right) \downarrow A_{7}=L\left(A_{7}\right) \oplus V_{A_{7}}\left(\lambda_{4}\right)$.

$L\left(E_{6}\right) \downarrow A_{1} A_{5}=L\left(A_{1} A_{5}\right) \oplus\left(V_{A_{1}}\left(\lambda_{1}\right) \otimes V_{A_{5}}\left(\lambda_{3}\right)\right)$

$L\left(E_{6}\right) \downarrow A_{2} A_{2} A_{2}=L\left(A_{2} A_{2} A_{2}\right) \oplus V_{A_{2} A_{2} A_{2}}\left(\left(\lambda_{1}, \lambda_{1}, \lambda_{1}\right)\right) \oplus V_{A_{2} A_{2} A_{2}}\left(\left(\lambda_{2}, \lambda_{2}, \lambda_{2}\right)\right)$. $L\left(F_{4}\right) \downarrow D_{4}=L\left(D_{4}\right) \oplus V_{D_{4}}\left(\lambda_{1}\right) \oplus V_{D_{4}}\left(\lambda_{3}\right) \oplus V_{D_{4}}\left(\lambda_{4}\right)$.

In the second $E_{6}$ case $V_{A_{2} A_{2} A_{2}}\left(\left(\lambda_{1}, \lambda_{1}, \lambda_{1}\right)\right)$ is just the tensor product of natural 3 -dimensional modules, one for each $A_{2}$ factor. In the last case the modules $V_{D_{4}}\left(\lambda_{i}\right)$ are the three 8-dimensional orthogonal representations. We also note that $V_{A_{k}}\left(\lambda_{i}\right)$ is the $i$-th wedge of a usual module.

Consider the action of an element $y_{i}$ on $L(G)$. We can write down the precise eigenvalues of $y_{i}$ on the natural module of the above classical group. Using this, we can determine the precise action on $L(G)$. In all but two cases using just the order of $y_{i}$ and the nature of the eigenvalues we find that $C_{L(G)}\left(y_{i}\right)$ has dimension equal to the rank of $G$ and that the fixed points are contained in the Lie algebra of the classical group indicated. One exception is $L=F_{4}(q)$ and $y_{i}=y_{2}$. Here $y_{i} \in G L_{4}(q)$ and on one of the orthogonal modules the $S L_{4}$ factor acts as $S O^{+}(6, q)$, leaving a 2 -space invariant. So when $q=2, y_{2}$ induces the identity on the 2 -space and hence $y_{2}$ has extra fixed points. This case is allowed for in the statement. The other exception is where $G=E_{6}>A_{1} A_{5}$ with $y_{i}=y_{2}=a b$, with $a$ in the $A_{5}$ factor and $b$ a noncentral element in the $A_{1}$ factor. Let $a_{0} \in\langle a\rangle$ have prime order for a primitive divisor of $q^{5}-\epsilon$. Then $\operatorname{dim}\left(C_{L(G)}\left(a_{o}\right)\right)=8$ and so $C_{G}\left(a_{o}\right)=A_{1} T_{5}$. Then $C_{G}\left(y_{2}\right) \leq C_{G}\left(a_{o}\right) \cap C_{G}(b)$, a maximal torus, as required.

Finally, we must show that $C_{G}\left(y_{i}\right)$ is connected. Let $\tilde{G}$ be the simply connected cover of $G, \tilde{y}_{i}$ a preimage of $y_{i}$, and $\tilde{T}$ the preimage of $T$. By II, 3.9, of [28] centralizers of semisimple elements in $\tilde{G}$ are connected. So we are done except perhaps when $G=E_{6}$ or $E_{7}$ and there is an element $\tilde{g} \in \tilde{G}$ such that $\tilde{y}_{i}^{\tilde{g}}=\tilde{y}_{i} z$, where $1 \neq z$ is a generator of $Z(\tilde{G})$. Here $z$ has order 3 (respectively 2) so $\tilde{g}$ centralizes $\tilde{t}=\tilde{y}_{i}^{3}$ (respectively $\left.\tilde{y}_{i}^{2}\right)$. However, arguing as above we find that $C_{\tilde{G}}(\tilde{t})=\tilde{T}$, which is a contradiction. (A slight modification is necessary in this argument when $L={ }^{2} E_{6}(2)$ with $y_{i}=y_{2}$. Here $\tilde{t}$ has order 11 and $C_{G}(\tilde{t})=A_{1} T_{5}$, which cannot contain such an element $\tilde{g}$.)

It will be convenient to settle the $F_{4}(2)$ case at this point. Here we take $x=y_{1}$ of order 17. Then Lemmas 7.2 .9 and 7.2 .7 imply that $C_{A}(x)=\langle x\rangle$, so we obtain the result provided there exists a sufficient number of classes of elements of different order. But there are already enough classes of unipotent elements (e.g. unipotents of type $A_{1}, A_{2}, F_{4}, B_{2}, C_{3}$ in the Bala-Carter notation; see pp.174-177 of [8] and note that these classes exist in all characteristics). 
Set $K_{i}=\left\langle y_{i}\right\rangle$ and $A_{i}=N_{A}\left(K_{i}\right) / C_{A}\left(K_{i}\right)$. In addition, write $q=p^{a}$, for $p$ a prime.

Lemma 7.2.10. (i) $C_{A}\left(y_{i}\right)=T_{i}$, a maximal torus of $G_{\sigma}$.

(ii) For $i=1,2,\left|A_{i}\right|$ divides $l_{i}$, where $\left(l_{1}, l_{2}\right)=(54 a, 54 a),(32 a, 14 a),(18 a, 20 a)$, or $(16 a, 32 a)$, according to whether $G=E_{8}, E_{7}, E_{6}$, or $F_{4}$, respectively. In the last case we can take $\left(l_{1}, l_{2}\right)=(8 a, 16 a)$, if $p \neq 2$.

Proof. Lemma 7.2 .9 shows that $C_{A}\left(y_{i}\right) \subseteq T\langle\delta, \tau\rangle$, where $T \subseteq C_{A}\left(y_{i}\right)$ is a maximal torus of $G_{\sigma}$ and $\delta, \tau$ are in the coset of a field and graph (or special graph) automorphism. The latter only occurs for $G=E_{6}$ or for $F_{4}$ with $p=2$. Suppose $a \in C_{A}\left(y_{i}\right) \backslash T_{i}$, which we may take to have prime power order. Then by Lemma 7.2.9] $a \in A \backslash G_{\sigma}$, and we may apply Lemma [7.2.7, If [7.2.7(i) holds, then $y_{i} \in G\left(q_{o}\right)$ and primitive divisor arguments rule out all cases except for $y_{1} \in E_{8}(q)$ and $y_{2} \in F_{4}(q)$. Then $y_{i}$ is contained in a maximal torus of $G\left(q_{o}\right)$ so 1.6 of [11] implies $\left|y_{i}\right| \leq\left(q_{o}+1\right)^{8},\left(q_{o}+1\right)^{4}$, respectively. This is impossible. If 7.2.7](ii) holds, then $L=E_{6}^{\epsilon}(q)$. Suppose $|a|=2$. Then by 1.1 of [11] either $p>2$ and $C_{G}(a)=F_{4}$ or $C_{4}$, or $p=2$ and $C_{G}(a)=F_{4}$ or $C_{F_{4}}(u)$ for $u$ a long root element. Taking fixed points under $\sigma$ and using order considerations we see that none of these have order divisible by $\left|y_{i}\right|$. Suppose $|a|>2$. If $p=2$, then $C_{L}\left(a^{2}\right)$ is contained in a canonical parabolic of $L$, say $P$, which is then stabilized by $a$. Order considerations show that the Levi factor must be of type $A_{5}(q)^{\epsilon}$. But then a must induce an involutory outer automorphism of this Levi factor centralizing the image of $y_{i}$. But there is no such automorphism. We have a similar contradiction if $p>2$, from consideration of the action of $a$ on the subsystem group $D=C_{L}\left(a^{2}\right)$, although here $D^{\prime}$ could be $A_{5}^{\epsilon}(q), A_{5}^{\epsilon}(q) A_{1}(q)$, or $D_{5}^{\epsilon}(q)$. The arguments are similar if 7.2.7(iii) holds. If $|a|=2$, then $y_{i}$ is in a maximal torus of ${ }^{2} F_{4}(q)$ and hence has order at most $(q+1)^{2}$, a contradiction. And if $|a|>2$, then $y_{i}$ is in a proper parabolic of ${ }^{2} F_{4}(q)$, giving a numerical contradiction. This proves (i).

Now consider (ii). We determine the normalizer $N_{G_{\sigma}}\left(K_{i}\right)$ from Carter [7. This normalizer modulo $T_{i}$ is the centralizer in the Weyl group of that element in the Weyl group determining the maximal torus. We use the information in Carter [7] to find this centralizer. We then obtain a bound for the full normalizer in $A$ by multiplying by the order of the group of field and graph (including special graph) automorphisms.

We are now in position to prove Proposition 7.2.8. Take $x$ to be a regular unipotent element. Lemmas 7.2.6 and 7.2.10 imply $d\left(x, y_{i}\right) \geq 3$ for $i=1,2$. Obviously this will also hold for any generators of $K_{i}$. So if we can find five such elements no two of which are conjugate in $A$, then we have the assertion.

Consider the orbits on the generators of $K_{i}$. We have $\phi\left(\left|K_{i}\right|\right) \geq \sqrt{\left|K_{i}\right|}$, where $\phi$ is the Euler function. So the number of nonconjugate generators is at least

$$
\sqrt{\left|K_{1}\right|} /\left|A_{1}\right|+\sqrt{\left|K_{2}\right|} /\left|A_{2}\right| \text {. }
$$

Suppose that this number is less than 5 . One checks that if $G=E_{8}$, then $q=2$ and otherwise $q \leq 4$, with the exceptions of $F_{4}(5)$ and $F_{4}(8)$.

It remains to work through these small values of $q$. For all cases other than $L(q)={ }^{2} E_{6}(2)$ one can use the precise numerical information to check that there are indeed at least five $A$-classes of generators of the groups $K_{i}$.

Assume $L={ }^{2} E_{6}(2)$. For this case we take $x=y_{1}$ to be an element of order 19 . So $x$ lies in a torus $T$ of $G_{\sigma}$ of order $2^{9}+1 / 2^{3}+1=19 \cdot 3$. Lemma 7.2.10 implies that 
$C_{A}(x)=T$. Suppose $t$ is an element of order 3 in this torus. From the construction of $y_{1}$ we see that $C_{G}(t)=A_{2} A_{2} A_{2}$ and $C_{L}(t)=P S U(3,8)$. Consequently, if we choose elements $y_{i}$ not conjugate to elements of $P S U(3,8)$, then $d\left(x, y_{i}\right) \geq 3$. But this is easy since there are sufficiently many unipotent classes with this property. This completes the proof of Proposition 7.2.8.

Lemma 7.2.11. Condition (Gen) holds if $L=G_{2}(q)(q \neq 2),{ }^{3} D_{4}(q)$, or ${ }^{2} F_{4}(q)^{\prime}$.

Proof. First assume $L=G_{2}(q)$. Then $L \geq A_{2}^{\epsilon}(q)$ which contains an element $x$ of order $q^{2}+\epsilon q+1$. We take $\epsilon=1$, unless this number is divisible by 3 , in which case we set $\epsilon=-1$. The maximal subsystem subgroups of $G$ are of type $A_{1} \tilde{A}_{1}$ and $A_{2}$ (also $\tilde{A}_{2}$ for $p=3$ ). Using this together with our choice of $\epsilon$ and the fact that $x$ is in no proper parabolic subgroup of $L$, we see that if $1 \neq g \in C_{G_{\sigma}}(x)$, then $C_{G}(g)$ is a torus. Also Lemma 7.2.7 implies $C_{A}(x)=\langle x\rangle$.

We can now take $y_{i}$ to be a nontrivial unipotent element multiplied by any semisimple element in its centralizer. It is easy to find enough choices. Using the Bala-Carter notation for unipotent elements we take $y_{1}=G_{2}$ (a regular unipotent element), $y_{2}=G_{2}\left(a_{1}\right)$ (a regular unipotent element in an $A_{2}$ subgroup), $y_{3}$ a unipotent element of type $A_{1}$, and $y_{4}=y_{3} z$, where $z$ is a semisimple element in $\tilde{A}_{1}$, the centralizer of the $A_{1}$ subgroup containing $y_{3}$. If $p \neq 3$ let $y_{5}$ be unipotent of type $\tilde{A}_{1}$. If $p=3$, then root elements for long and short roots are conjugate in $A$, but here there is an extra class of 3 -central elements and $y_{5}$ is taken as a representative of this class.

The cases $Y={ }^{3} D_{4}(q)$ and ${ }^{2} F_{4}(q)^{\prime}$ are handled similarly. We take $x$ to be a semisimple element of order $q^{4}-q^{2}+1$ or $q^{2}+q \sqrt{2 q}+q+\sqrt{2 q}+1$, respectively. These numbers are factors of $q^{4}-q^{2}+1$ and we argue as above that $C_{A}(x)$ is a torus and that no nonidentity element of this torus centralizes a nontrivial unipotent element. So again we choose elements $y_{i}$ with nontrivial unipotent part. The existence of such elements follows easily as in the $G_{2}$ case, from the containments $S L(2, q) \cdot S L\left(2, q^{3}\right)<{ }^{3} D_{4}(q)$ and ${ }^{2} B_{2}(q) \cdot{ }^{2} B_{2}(q)<{ }^{2} F_{4}(q)$, except when $L=$ ${ }^{2} F_{4}(2)^{\prime}$. Here $x$ has order 13 . Then $C_{A}(x)=\langle x\rangle$ and we need only show that there are at least five $A$-classes within $L$ of elements having order not dividing 13 . This can be easily checked from the ATLAS.

This leaves the rank 1 Suzuki and Ree groups.

Lemma 7.2.12. Condition (Gen) holds if $L={ }^{2} G_{2}(q)(q>3)$ or ${ }^{2} B_{2}(q)(q>2)$.

Proof. First assume $L \neq{ }^{2} B_{2}(8)$. Let $x$ be an element of order $q+\sqrt{3 q}+1$ or $q \pm \sqrt{2 q}+1$ according to whether $L={ }^{2} G_{2}(q)$ or ${ }^{2} B_{2}(q)$. As in other cases $C_{A}(x)=$ $\langle x\rangle=T$, a torus, and $C_{L}(t)=T$ for each nonidentity element $t \in T$. It only remains to exhibit appropriate elements $y_{1}, \ldots, y_{5}$.

For ${ }^{2} G_{2}(q)$ this is easy. Let $y_{1}, y_{2}, y_{3}$ be elements of order $3,3,9$, respectively. We choose these elements so that $C_{L}\left(y_{1}\right)$ is a Sylow 3 -group, while $C_{L}\left(y_{2}\right)$ contains an involution $t$ (recall that $\left.{ }^{2} G_{2}(q) \geq L_{2}(q) \times\langle t\rangle\right)$. Now let $y_{4}=y_{2} t$ and let $y_{5}$ be an element of order $q-1$.

Suppose $L={ }^{2} B_{2}(q)$, with $q>8$. We note that 5 divides

$$
q^{2}+1=(q+\sqrt{2 q}+1)(q-\sqrt{2 q}+1) .
$$

Choose $x$ so that $|x|$ is the factor not divisible by 5 . Now choose elements $y_{1}, \ldots, y_{5}$ such that $\left|y_{1}\right|=2,\left|y_{2}\right|=4,\left|y_{3}\right|=q-1,\left|y_{4}\right|=5$, and $\left|y_{5}\right|=q \pm \sqrt{2 q}+1$, where we choose signs so that $|x| \cdot\left|y_{5}\right|=q^{2}+1$. The result follows. 
Finally, consider $L={ }^{2} B_{2}(8)$. Let $x$ have order 13. Then $C_{A}(x)=\langle x\rangle$. Then choose $y_{1}, y_{2}, y_{3}, y_{4}, y_{5}$ as elements of order $2,4,4,5,7$, noting that there are two classes of elements of order 4 in $A$ (see ATLAS).

\section{The classical groups}

Lemma 7.2.13. Condition (Gen) holds in each of the following situations:

(i) $L=P S p(2 n, q)$ for $n \geq 2$ and $L \neq P S p(4,2)$.

(ii) $L=P S L(n, q)$ for $n \geq 3$ and $L \neq P S L(3,2), P S L(3,4)$.

(iii) $L=P S U(n, q)$ for $n \geq 3$.

Proof. For $L=P S p(2 n, q), P S L(n, q)$, or $\operatorname{PSU}(n, q)$ for $n$ odd, let $x$ be a generator of the image in $L$ of a cyclic irreducible torus (Singer cycle) of $S p(2 n, q), S L(n, q)$ or $S U(n, q)$, respectively. Then $|x|=q^{n}+1 /(2, q-1), q^{n}-1 /(q-1)(n, q-1)$, or $q^{n}+1 /(q+1)(n, q+1)$, respectively. If $L=P S U(n, q)$ with $n$ even, take $x$ of order $q^{n}-1 /(q+1)(n, q+1)$, except for the cases $(n, q)=(4,3)$ and $(6,2)$, which we postpone until later in the proof. Also, $P S U(4,2) \cong P S p(4,3)$ and we will work with $\operatorname{PSp}(4,3)$.

We claim $C_{A}(x)$ is a maximal torus of $G_{\sigma}$, with $\langle x\rangle$ of index $(2, q-1)$, $(n, q-1)$, or $(n, q+1)$, respectively. We first use the action on the usual module to argue that $C_{G_{\sigma}}(x)=T$, a maximal torus. Some care must be taken in this as we are working in the simple group rather than the linear group. In cases where the classical group has a nontrivial center let $y \in\langle x\rangle$ be an element of prime order for a primitive divisor of $|x|$, except for $L=P S U_{n}(q)$ with $n$ even where we set $y=x^{(n, q+1)}$. Then $C_{L}(y)$ is covered by the centralizer in the corresponding linear group and $y$ has distinct eigenvalues on the natural module. Thus $C_{G_{\sigma}}(y)=T$ and hence $C_{G_{\sigma}}(x)=T$.

If $C_{A}(x)>T$, then $x$ is centralized by an element $a \in A \backslash G_{\sigma}$ such that $a G_{\sigma}$ has prime order and $a$ has prime power order. Lemma 7.2.7 together with primitive divisor arguments reduce us to the case where $a G_{\sigma}=\tau G_{\sigma}$ for $\tau$ a graph or graph field automorphism of $P S L_{n}^{\epsilon}(q)$ or an involutory special graph automorphism of $P S p(4, q)$. If $a$ is in the coset of a special graph automorphism, then 7.2.7(iii) shows that either $x$ is contained in a parabolic subgroup of $P S p(4, q)$ or it is in ${ }^{2} B_{2}(q)$. The former is clearly impossible. In the latter case $q^{2}+1$ divides the order of ${ }^{2} B_{2}(q)$, but we see from the determination of conjugacy classes in [30] that semisimple elements of ${ }^{2} B_{2}(q)$ have order at most $q+\sqrt{2 q}+1$, a contradiction. Hence, $L=P S L_{n}^{\epsilon}(q)$. Let $D$ be as in 7.2.7(ii). If $D$ is reductive, then $D_{\sigma}$ cannot contain $x$ (although it may contain an element of order a primitive prime divisor of $|x|$. For example this happens in certain cases where $D$ is a symplectic group or an orthogonal group. It also happens when $\tau$ is a graph field automorphism of $L=P S L(n, q)$ for $n$ odd and $\left.G_{\delta} \cong P G U(n, \sqrt{q})\right)$.

Now suppose $D$ is parabolic. This forces $p=2$ and since $x$ does not centralize an involution of $L$ we must have $|a|=2$. The parabolic case arose here when $D=C_{G}(a)$ had nontrivial unipotent radical. But this only occurs for $n$ even where $D$ is the centralizer of a root element in the corresponding symplectic group. But then order considerations show $x \notin D_{\sigma}$. We have now proved the claim.

Choose elements as follows. Consider the subgroups $S p(2 r, q) \times S p(2 n-2 r, q) \leq$ $S p(2 n, q)$ for $1 \leq r \leq n ; S L(r, q) \times S L(n-r, q) \leq S L(n, q)$ for $2 \leq r \leq n$; and $S U(r, q) \times S U(n-r, q) \leq S U(n, q)$ for $2 \leq r \leq n$. Let $u_{r}$ be a regular unipotent 
element of the first factor and $s_{n-r}$ any semisimple element in the second factor. Set $d_{r}=u_{r} s_{n-r}$.

We claim that $C_{A}\left(d_{r}\right) \cap C_{A}(x)=1$. Suppose $1 \neq g$ is in the intersection. First note that $C_{A}\left(d_{r}\right)=C_{A}\left(u_{r}\right) \cap C_{A}\left(s_{n-r}\right)$. By the claim $g \in T$ and so $g$ is a semisimple element of $G$. Now $C_{G}(g)$ is a $\sigma$-invariant (reductive) subsystem subgroup containing both $x$ and $u_{r}$. Using the fact that $x \in C_{G}(g)$, together with primitive divisor arguments, we find that there are few possibilities for $C_{L}(g)$. In particular, there is a factorization $n=a b$ and either $C_{G}(g)_{\sigma}$ is irreducible on the natural module so that $C_{G}(g)_{\sigma}^{\prime} \in\left\{P S p\left(2 a, q^{b}\right), P S L\left(a, q^{b}\right), P S U\left(a, q^{b}\right)\right.$ (b odd) $\}$, or $L=P S U(n, q)$ with $n$ even and $C_{G_{\sigma}}(g)^{\prime}=P S L\left(a, q^{b}\right)$. In each case $C_{G}(g)^{\prime}$ is a commuting product of several isomorphic simple groups with $\langle\sigma\rangle$ permuting the components transitively. But now consider the embedding of $u_{r}$ in this centralizer. On the one hand $u_{r}$ is a diagonal element in the commuting product. On the other hand $u_{r}$ has a single nontrivial Jordan block on the natural module. This is a contradiction.

We have shown that $d\left(x, d_{r}\right) \geq 3$. It remains to show that there are enough such elements. In the symplectic case this is clear if $n \geq 5$, since here we can simply take $y_{i}=u_{i}$ for $1 \leq i \leq 5$. For smaller symplectic groups we take as many unipotent elements as possible and then adjust them by semisimple factors. For example if $L=P S p(4, q), q>4$, we set $y_{1}=u_{2}$ (a regular unipotent element), $y_{2}=u_{1}$ and $y_{i}=u_{1} z_{i}$ for $3 \leq i \leq 5$, where $z_{3}, z_{4}, z_{5}$ are nonidentity semisimple elements of $S p(2, q)$ of different orders. If $L=P S p(4,4)$, then $|x|=17$. Then $C_{A}(x)=\langle x\rangle$ so we can take $y_{1}, \ldots, y_{5}$ to be any nonconjugate elements of order different from 17 . Suppose $L=P S p(4,3)$. Here $|x|=5$ and $C_{A}(x)=\langle x, t\rangle$, where $t$ is an involution with $C_{L}(t)=S_{6}$ (regard $L$ as $P S U(4,2)$ and $C_{L}(t)$ as $S p(4,2)$ ). From ATLAS we see that $L$ contains four $A$-classes of elements of order 6 only two of which reside in $C_{L}(t)$. Also root elements of order 3 cannot lie in $C_{L}(t)$. Consequently we can take $y_{1}, \ldots, y_{5}$ as elements of order $3,6,6,9,12$, respectively.

Now consider the cases $L=P S L(n, q), P S U(n, q)$. As noted earlier, $P S U(4,2) \cong$ $P S p(4,3)$ was handled in the previous paragraph. For $n \geq 6$ we just use $u_{2}, \ldots, u_{6}$. For smaller values of $n \geq 4$ multiply the $u_{i}$ by semisimple elements as above. The details are quite easy, except for the case $\operatorname{PSL}(4,3)$. Here we take $y_{1}=u_{4}, y_{2}=$ $u_{2}, y_{3}=u_{2} s, y_{4}=u_{2} t, y_{5}=z$, where $s, t$ are elements of order 2,4 centralizing $u_{2}$ and $z$ has order 13 .

Next we consider the previously excluded cases $L=P S U(4,3), L=P S U(6,2)$, where we can argue using the natural module and ATLAS. In these cases take $x$ to be a semisimple element of order 7,11 respectively. We then find that $C_{A}(x)=$ $\langle x\rangle \times Z$, where $Z$ is cyclic of order $q+1$ with $Z \cap L=1$. If $1 \neq z \in Z$, then $C_{L}(z)=S U(n-1, q)$. We can now choose elements $y_{1}, \ldots, y_{5}$ as follows. If $L=$ $P S U(6,2)$, let $y_{1}$ be a regular unipotent element, $y_{2}$ a diagonal involution in three copies of $S U(2,2), y_{3}$ an element of order 7 (in a subgroup $L(3,4)$ ), $y_{4}$ a regular unipotent element in $L(3,4)$, and $y_{5}$ an element of order 10. If $L=P S U(4,3)$, let $y_{1}$ be a regular unipotent element, $y_{2}$ an element of order $5, y_{3}$ an element of order 6 (there are three classes, choose one not represented in $S U(3,3)), y_{4}$ an element of order 4 in $P S L(2,9)$, and $y_{5}$ a unipotent element of $P S L(2,9)$. From what has been established so far, we obtain the result in both these cases.

We use a slightly different argument for $L=P S L(3, q), P S U(3, q)$ as there are fewer classes of unipotent elements. Let $x$ be as before and set $y_{1}=u_{3}$ (a regular unipotent element) and $y_{2}=u_{2}$ (a root element). We are assuming $q \neq 2,4$ 
for $\operatorname{PSL}(3, q)$ so we can take $y_{3}=u_{2} s$, for $1 \neq s$ of order dividing $q-1, q+1$, respectively. There is a cyclic maximal torus of $S L(3, q)$ and $S U(3, q)$ of order $q^{2}-1$ and $y_{4}, y_{5}$ are taken as images in $P S L(3, q)$ (resp. $P S U(3, q)$ ) of members of this torus. We need to verify that there are two such classes and that they have distance at least 3 from $x$.

The latter statement is established as above, by looking at the full centralizer of an element centralizing both $x$ and $y_{i}$ for $i=4,5$. For the former, first note that $A$-fusion in the torus is controlled by the normalizer, which induces a group of order $4 a$, where $q=p^{a}$. Hence, in order to get two nonidentity classes it will suffice that $\left(q^{2}-4\right) / 3 \cdot 4 a \geq 2$. This holds provided $q \neq 2,3,4,5,8$. If $q=3$ or 5 , the torus in question has an element of order 8 so we can choose elements $y_{4}, y_{5}$ of order 4 and 8. Similarly we can choose elements of different order when $q=8$. So we have the result, except for the case $P S U(3,4)$. Here we note that there are at least two classes of elements of order 5 and we take $y_{4}, y_{5}$ such elements.

Lemma 7.2.14. Let $L=P S L(2, q)$. Condition $(G e n)$ holds except when $q=$ $5,7,8,9,11,16$, or 27 .

Proof. Let $x$ be a nonidentity unipotent element, so that $C_{A}(x)=U\langle\delta\rangle$ for $\delta$ a field automorphism. Then 7.2.7)(i) implies $d(x, y) \geq 3$ for any semisimple element $y \in L$ such that $y$ is not centralized by a nontrivial field automorphism of $L$. In particular this will hold if $y$ is taken as a generator of a cyclic maximal torus of order $(q-1) / d$ or $(q+1) / d$, for $d=(2, q-1)$. Using the Euler $\phi$-function we see that there are at least $\sqrt{(q-1) / d}, \sqrt{(q+1) / d}$ such elements, respectively. Under the action of the normalizer of this torus the generators fall into classes of length $2 a$. Consequently, we can find at least five such conjugacy classes provided

$$
2(\sqrt{(q-1) / d}) / 2 a \geq 5 .
$$

So the assertion holds provided $q-1 \geq 25 d a^{2}$. A direct check shows that this condition is satisfied except for the following cases:

$$
\begin{gathered}
p=2, a \leq 11 ; \quad p=3, a \leq 7 ; \quad p=5, a \leq 4 ; \quad p=7, a \leq 3 ; \\
p=11,13, a \leq 2 ; \quad q=17,19, \ldots, 47 .
\end{gathered}
$$

All cases, except $P S L(2,13)$ and $P S L(2,25)$, can be checked directly, using precise information on the number of generators of the tori. For example, if $L=$ $P S L(2,64)$, then there are 48 generators of a torus of order 65 , falling into four orbits under the normalizer in $A$. So take representatives of these orbits together with a generator of a torus of order 63 . The cases can all be settled in this way and details are left to the reader. Consider $P S L(2,13)$. Choose $y_{1}, \ldots, y_{5}$ of order $2,3,6,7,7$ (note that $A$ has three classes of elements of order 7 ). Since $C_{A}(x)=\langle x\rangle$, the result follows. Finally, consider $\operatorname{PSL}(2,25)$. Here we take $x$ to be an element of order 13. Then $C_{A}(x)$ has order 26 and the involution in this group has $L$ centralizer equal to $\langle x\rangle$. So just choose elements $y_{1}, \ldots, y_{5}$ of order $3,4,5,6,12$ to get the result.

The final lemma of this subsection deals with orthogonal groups. We ignore odd dimensional orthogonal groups in even characteristic, as these were handled previously as symplectic groups.

Lemma 7.2.15. Let $L=P S O^{\epsilon}(n, q)^{\prime}$ for $n \geq 7$. Then (Gen) holds unless $L=$ $\mathrm{PSO}^{+}(8,2)$. 
Proof. For later reference we first note that $S O^{\epsilon}(2 k, q)$ contains an element of order $q^{k}-\epsilon$, which generates a maximal torus. For $p>2$, this element is not contained in $S O^{\epsilon}(2 k, q)^{\prime}$, although the derived group does contain the square of the element.

First assume that $n=2 k+1$ is odd, so that there are no graph automorphisms. Take $x$ to be a regular unipotent element. Then $C_{A}(x)=U\langle\delta\rangle$, for $\delta$ a field automorphism. It will suffice to find a sufficient number of semisimple elements $y$ centralized by no element of $U\langle\delta\rangle$. We note that 7.2.7 $\mathrm{i}$ ) shows that elements of $U\langle\delta\rangle \backslash U$ have fixed points on $G$ of the form $G\left(q_{o}\right)$ for $q_{o}<q$ (as usual we identify an automorphism of $L$ with an extension to $G)$.

Temporarily exclude $L=P S O(7,3)^{\prime}$. In the remaining cases choose elements as follows. Let $y_{1} \in S O^{+}(2 k, q)^{\prime}$ have order $\left(q^{k}-1\right) / 2$ and $y_{2} \in S O^{-}(2 k, q)^{\prime}$ have order $\left(q^{k}+1\right) / 2$. Similarly, choose elements $a_{3}, a_{4}$ of order $\left(q^{k-1}+1\right) / 2,\left(q^{k-1}-1\right) / 2$, respectively, in groups $S O^{-}(2 k-2, q)^{\prime}, S O^{+}(2 k-2, q)^{\prime}$ and let $b_{3}, b_{4}$ be nontrivial semisimple elements of $C_{L}\left(S O^{ \pm}(2 k-2, q)^{\prime}\right) \cong S O(3, q)^{\prime}$. Set $y_{3}=a_{3} b_{3}$ and $y_{4}=$ $a_{4} b_{4}$. If $k$ is odd, let $y_{5}$ be a power of $y_{2}$ of order $\left(q^{k}+1\right) /(q+1)$, and if $k$ is even, let $y_{5}=a_{5} b_{5}$, where $a_{5} \in\left\langle a_{3}\right\rangle$ has order $\left(q^{k-1}+1\right) /(q+1)$ and $b_{5}=b_{3}$.

We claim that $C_{G_{\sigma}}\left(y_{i}\right)$ is a maximal torus for $1 \leq i \leq 5$. The claim is equivalent to the assertion that $C_{G}\left(y_{i}\right)$ is a maximal torus. If this is not the case, then $C_{G}\left(y_{i}\right)$ contains a simple component which would centralize the preimage of $y_{i}$ in its action on the natural module, hence stabilize each eigenspace. However, by the choice of $y_{i}$ each eigenspace either has dimension 1 or the eigenvalue is -1 with corresponding eigenspace being nondegenerate of dimension at most 2 . In any case the eigenspace can afford only the trivial action of the simple factor, a contradiction. This gives the claim and then Lemma 7.2.7(i) implies $C_{A}\left(y_{i}\right)$ is torus. Then the second paragraph gives $d\left(x, y_{i}\right) \geq 3$ for $i=1, \ldots, 5$.

If $L=P S O(7,3)^{\prime}$, set $y_{1}, y_{2}, y_{3}, y_{4}, y_{5}$ elements of order $10,13,20,14,7$, respectively. Using ATLAS we see that none of these elements is centralized by a nontrivial unipotent element of $L$. On the other hand, $C_{A}(x)=U$, a unipotent group. So the result holds here as well.

Now suppose $n=2 k$. First assume $k \geq 5$. We again take $u$ to be a regular unipotent element, but here we must be more careful as $C_{A}(u)=U\langle\tau, \delta\rangle$, where $\tau$ is a graph automorphism which centralizes large parts of maximal tori. Let $y_{1}$ be the image in $L$ of an element of $S O^{\epsilon}(2 k, q)^{\prime}$ of order $\left(q^{k}-\epsilon\right) / d$, where $d=1$ or 2 , according to whether $q$ is even or odd.

There exist subgroups $S O^{-\epsilon}(2 k-4, q) \times S O^{-}(4, q)$ and $S O^{\epsilon}(2 k-4, q) \times S O^{+}(4, q)$ of $S O^{\epsilon}(2 k, q)$. From the first subgroup we take $y_{2}=a_{2} b_{2}$ and $y_{3}=a_{3} b_{3}$, where $a_{2}=a_{3}$ is the image in $\operatorname{PSO}^{\epsilon}(2 k, q)^{\prime}$ of an element of order $q^{k-2}+\epsilon$, while $b_{2}, b_{3}$ are images of elements of order $q^{2}-1, q^{2}+1$, respectively. For $p$ odd, the elements $a_{i}, b_{i}$ do not lie in the derived group of $\operatorname{PSO}^{\epsilon}(n, q)$, but their product does.

Let $y_{4}=a_{4} b_{4}$ be in the second group, with $a_{4}$ of order $q^{k-2}-\epsilon$ and $b_{4}$ of order $q^{2}-1$ (if $q=2$ choose $b_{4}$ to be in one of the $S L(2,2)$ factors of $S O^{+}(4,2)$ ). Each of $y_{2}, y_{3}, y_{4}$ are products of two elements and these elements may not have relatively prime orders. So the order of $y_{i}$ may be less than that of the product of the orders of the factors. More important than the order is the action of a preimage of $y_{i}$ on the orthogonal module and this action is clear from the description.

We require one more element. If $q^{k}-\epsilon$ has a primitive prime divisor of order less than $\left|y_{1}\right|$, then we can take $y_{5}$ to be an element of this order. If there is no such element, then either (i) $q^{k}-\epsilon=p^{2^{s}}+1$; or (ii) $q^{k}-\epsilon=2^{k}-1=r$, with $r$ prime; or (iii) $q^{k}-\epsilon=3^{k}-1=2 r$ with $r$ prime. In the last two cases there 
are $r-1$ generators of $\left\langle y_{1}\right\rangle$ and we can choose $y_{5}$ as a generator not $A$-conjugate to $y_{1}$ (eigenvalue arguments show that $N_{A}\left(\left\langle y_{i}\right\rangle\right) / C_{A}\left(y_{i}\right)$ has order at most $2 k$ ). If (i) holds we can factor $q^{k-2}+\epsilon$, take an element $a_{5} \in\left\langle a_{3}\right\rangle$ of order a primitive prime divisor and then set $y_{5}=a_{5} b_{5}$, where $b_{5}=b_{3}^{2}$ (we use the square to obtain an element in $\left.\mathrm{SO}^{-}(4, q)^{\prime}\right)$.

We claim that for $1 \leq i \leq 5, C_{A}\left(y_{i}\right)$ is a torus of $G_{\sigma}$. As above, eigenspace arguments show that $C_{G_{\sigma}}\left(y_{i}\right)$ is a torus. Next, 7.2.7)(i) and primitive divisor arguments reduce consideration to elements in the coset of a graph automorphism. Let $a$ be as in 7.2.7 (ii), with $|a|$ a power of 2 . Note that $a$ is in the image of the full orthogonal group. If $p=2$, then $a$ is an involution, as $y_{i}$ centralizes no involution in $G_{\sigma}$. Then $a$ is of type $b_{j}$ in the notation of [2] and 8.7 of [2] shows that semisimple elements in $C_{L}(a)$ have fixed points on the orthogonal module. This is a contradiction as $y_{i}$ has no fixed points.

Suppose $p$ is odd. Let $\hat{a}$ and $\hat{y}_{i}$ denote preimages of $a, y_{i}$ in $S O(n, q)$. Then $\hat{a}$ centralizes $\hat{y}_{i}$ modulo the center, so $\hat{a}$ centralizes $\hat{y}_{i}^{2}$. Consider the eigenspaces of $\hat{a}$. If $\beta$ is an eigenvalue other than \pm 1 , then $\beta^{-1}$ must also be an eigenvalue of equal multiplicity, as otherwise $\hat{a}$ would not preserve the orthogonal form. As $\operatorname{det}(\hat{a})=-1$, we see that the eigenspace for eigenvalue -1 must be nondegenerate of odd multiplicity. However, by the choice of $y_{i}$ we see that $\hat{y}_{i}^{2}$ leaves invariant no nondegenerate subspace of odd dimension. This establishes the claim and the result follows.

We are left with the cases $L=P S O^{\epsilon}(8, q)^{\prime}$, excluding $L \cong P S O^{+}(8,2)$. First assume $q>5$ for $\epsilon=1$ and $q>3$ for $\epsilon=-1$.

Let $x$ be a regular unipotent element. Choose $y_{1}$ to be the image in $L$ of an element of order $\left(q^{4}-\epsilon\right) / d$ in $S O^{\epsilon}(8, q)^{\prime}$ for $d=1$ or 2 . Write $q=p^{a}$.

We first claim that $C_{A}\left(y_{1}\right)$ is a maximal torus of $G_{\sigma}$ and that $N_{A}\left(\left\langle y_{1}\right\rangle\right) / C_{A}\left(\left\langle y_{1}\right\rangle\right)$ has order dividing $16 a$.

Using the description of $\left\langle y_{1}\right\rangle$ as a maximal torus, we see that it is normalized by a group of field automorphisms of $L$ of order $a$. Next argue that for $\epsilon=1$ no element in the coset of a nontrivial triality graph automorphism can normalize $\left\langle y_{1}\right\rangle$. Indeed, triality morphisms transitively permute the 8-dimensional orthogonal representations, whereas if $v \in\left\langle y_{1}\right\rangle$ has order a primitive prime divisor of $\left|y_{1}\right|$, then $\hat{v} \in S L_{4}(q)$ has no fixed points on two of these modules, but a 2-dimensional fixed point space on the third.

The usual arguments show that $C_{A}\left(y_{1}\right)$ is a maximal torus of $G_{\sigma}$ and $N_{G_{\sigma}}\left(\left\langle y_{1}\right\rangle\right)$ induces a group of order 8 (the centralizer of an element of order 4 or 8 in the Weyl group, according to whether $\epsilon=1$ or -1 ). The claim now follows from the structure of $A$.

At this point we argue as in 7.2.14 that we can choose $y_{2}, \ldots, y_{5}$ as nonconjugate generators of $\left\langle y_{1}\right\rangle$. For $q>11$ this follows easily from numerical estimates, and in smaller cases one uses precise information on the number of generators. The result follows.

This leaves us with several small cases. If $L \cong P S O^{-}(8,2)$, let $x \in L$ be an element of order 17 . Then $C_{A}(x)=\langle x\rangle$ and so we can take $y_{1}, \ldots, y_{5}$ as any classes corresponding to elements of $L$ having order other than 17 .

If $L \cong P S O^{-}(8,3)$, let $x \in L$ be an element of order 41 . Then $C_{A}(x)=\langle x, t\rangle$, for $t$ an involution ( $t$ is in a maximal torus of the full orthogonal group). The only possibility is that $C_{L}(t)=L_{2}(81)$. This centralizer is diagonal in a group $A_{1}^{4}<G=$ $D_{4}$. Let $y_{1}, \ldots, y_{5}$ be unipotent elements of type $A_{1}, A_{1} A_{1}, A_{1} A_{1} A_{1}, A_{3}, D_{4}$ (in the 
Bala-Carter notation), respectively. From the action on the orthogonal module we see that none of these is represented in $C_{L}(t)$.

Assume $L=P S O^{+}(8,5)$. Let $x$ (a regular unipotent element) and $y_{1}$ be as before. Here $\left\langle y_{1}\right\rangle$ has 48 generators; thus by the above claim there are at least three orbits on generators so we take additional orbit representatives $y_{2}, y_{3}$. Now set $y_{4}=a_{4} b_{4}$, for $a_{4} \in S O^{-}(6,5)$ of order $5^{3}+1$ and $b_{4} \in S O^{-}(2,5)$ of order $5+1$, and set $y_{5}=a_{5} b_{5}$, for $a_{5}=a_{4}^{6}$ and $b_{5}=a_{4}^{2}$. The earlier arguments show that $C_{A}\left(y_{i}\right)$ is a torus for each $i$ and the result follows.

Finally, consider $L=\mathrm{PSO}^{+}(8,3)^{\prime}$. Here we take $x$ to be an element of order 20 , realized as an element of order 5 in $S O^{-}(4,3)$ times an element of order 4 in a commuting $S O^{-}(4,3)$. Write $x=x_{5} x_{4}$, a product of commuting elements of order 5,4 , respectively. Then $C_{A}(x)=C_{A}\left(x_{5}\right) \cap C_{A}\left(x_{4}\right)$. Each of $C_{A}\left(x_{5}\right)$ and $C_{A}\left(x_{4}\right)$ contains a unique subgroup $S O^{-}(4,3)^{\prime} \cong P S L(2,9)$. Hence $C_{A}(x)$ normalizes each factor of $S O^{-}(4,3)^{\prime} \times S O^{-}(4,3)^{\prime}$. We now argue that $\left|C_{A}(x)\right|=80$ and that if $1 \neq r \in C_{A}(x)$ has prime order, then either $r$ has $O^{+}(8,3)$-centralizer contained in $O^{-}(4,3) \cdot O^{-}(4,3)$ or $r$ is an involution with $C_{L}(r)^{\prime}=S O^{ \pm}(6,3)^{\prime}$. Let $y_{1}, y_{2}, y_{3}$ be unipotent elements of types $A_{1} A_{1} A_{1}, D_{4}, D_{4}\left(a_{1}\right)$, respectively. None of these elements can lie in a subgroup $S O^{ \pm}(6,3)$ or $O^{-}(4,3) \cdot O^{-}(4,3)$. There is a subgroup $A_{1} A_{1} A_{1} A_{1}$ in $G$, hence a subgroup $S L(2,3)$ of $L$ centralizing $y_{1}$. So take $y_{4}=y_{1} a, y_{5}=y_{1} b$, where $a, b \in S L_{2}(3)$ are elements of order 2 and 4 , respectively. The result follows.

7.3. The nongeneric cases. The purpose of this subsection is to show that when $L$ is one of the groups

$$
P S L(2, q), \quad q=5,7,8,9,11,16,27, \quad P S L(3,4) \text { or } P S O^{+}(8,2),
$$

then $G$ satisfies Property $\left(3 \frac{1}{2}\right)$.

Lemma 7.3.1. Assume $L \cong P S L(2,8)$. Then $G$ has Property $\left(3 \frac{1}{2}\right)$.

Proof. Let $B_{1}, B_{2}, B_{3} \subseteq L$ be the $\operatorname{Aut}(L)$ conjugacy classes of elements of order $3,7,9$, respectively. Let $C_{1}, \ldots, C_{4} \subseteq L$ be the Aut $(L)$ conjugacy classes of elements of order $3,7,9,2$, respectively.

We show that the hypotheses (c) of Proposition 7.1 .8 hold; to show hypothesis (c2), we prove that the assumptions in part (a) or (b) of Lemma 7.1.6 are satisfied. We first claim that:

$\left(^{*}\right)$ For all $u, v \in \bigcup_{i=1}^{3} B_{i}$ such that $\langle u\rangle \cap\langle v\rangle=1, d_{\mathrm{Aut}(L)}(u, v)>3$.

Indeed, assume $u \in B_{1}$ is of order 3 . Then it is easy to check that every element $w \in \Delta_{\operatorname{Aut}(L)}^{\leq 3}(u)$ must either centralize $u$ or one of the nine involutions in $N_{L}(\langle u\rangle) \cong$ $D_{18}$. Thus if in addition $w \in L$, then either $w \in C_{L}(u) \cong \mathbb{Z}_{9}$ or $w$ is an involution. Also since $\langle u\rangle \cap\langle v\rangle=1, v$ cannot belong to $C_{L}(u)$. If $u \in B_{2}$, then $\left(^{*}\right)$ is obvious, and if $u \in B_{3}$, then $u^{3} \in B_{1}$ and we saw that $d_{\operatorname{Aut}(L)}\left(u^{3}, v\right)>3$, and hence also $d_{\text {Aut }(L)}(u, v)>3$. This shows $(*)$, and hence hypothesis (a) of Lemma 7.1.6, for $\mathcal{B} \in\left\{B_{1}, B_{2}, B_{3}\right\}$ and $\mathcal{C} \in\left\{C_{1}, C_{2}, C_{3}\right\}$, holds. It remains to show that:

(**) Let $\mathcal{C}$ be the class of involutions of $L$. Then for each $j \in\{1,2,3\}$, if we set $\mathcal{B}=B_{j}$, then the pair $\mathcal{B}, \mathcal{C}$ satisfies hypothesis (b) of Lemma 7.1.6

Indeed let $v \in \mathcal{C}$ be an involution and $1 \neq t \in C_{\mathrm{Aut}(L)}(v)$. We will find $u^{\prime} \in \mathcal{B}$ such that

$$
d_{\mathrm{Aut}(L)}\left(u^{\prime}, v\right) \geq 3 \leq d_{\operatorname{Aut}(L)}\left(u^{\prime}, t\right)
$$


note that we may assume that the order of $t$ is a prime. Assume $t$ is an involution. The reader may easily verify that we can choose $u^{\prime} \in \mathcal{B}$ not inverted by $t$ or $v$ and that such a $u^{\prime}$ satisfies (i). Assume that $t$ has order 3 ( $t$ is an outer automorphism). Note that $C_{L}(t) \cong S_{3}$ and so any $u^{\prime} \in B_{1}$ such that $u^{\prime} \notin C_{L}(t)$ is at distance $\geq 3$ from $t$ in $\Delta_{\text {Aut }(L)}$. Thus any $u^{\prime} \in B_{1}$ not inverted by $v$ satisfies (ii). A similar argument shows that any element $u^{\prime} \in B_{2} \cup B_{3}$, not inverted by $v$, satisfies (ii).

Lemma 7.3.2. Assume $L \cong P S L(2,7)$. Then $G$ has Property $\left(3 \frac{1}{2}\right)$.

Proof. The proof here is similar to the proof of Lemma 7.3.1. Let $B_{1}, B_{2}, B_{3} \subseteq$ $L$ be the $\operatorname{Aut}(L)$ conjugacy classes of elements of order 3,4,7, respectively. Let $C_{1}, \ldots, C_{4} \subseteq L$ be the Aut $(L)$ conjugacy classes of elements of order $3,4,7,2$, respectively.

We show that the hypotheses in (c) of Proposition 7.1.8 hold; again, to show hypothesis (c2), we prove that the assumptions in part (a) or (b) of Lemma 7.1.6 are satisfied. We claim that:

(*) (1) For $u \in B_{3}$ and $1 \neq v \in L$, with $\langle u\rangle \neq\langle v\rangle$, we have $d_{\operatorname{Aut}(L)}(u, v)>3$.

(2) For $u \in B_{1}$, we have $\left|B_{1} \cap \Delta_{\operatorname{Aut}(L)}^{\leq 3}(u)\right|=8$ and $\left|B_{2} \cap \Delta_{\operatorname{Aut}(L)}^{\leq 3}(u)\right|=6$.

(3) For $u \in B_{2}$, we have $\left|B_{1} \cap \Delta_{\operatorname{Aut}(L)}^{\leq 3}(u)\right|=8$ and $\left|B_{2} \cap \Delta_{\operatorname{Aut}(L)}^{\leq 3}(u)\right|=10$.

Part (1) of (*) is obvious. For part (2) of (*) assume $u \in B_{1}$ is of order 3 . Let $t \in C_{\mathrm{Aut}(L)}(u)$ be the unique involution. Then every element at distance $\leq 3$ from $u$ in $\Delta_{\operatorname{Aut}(L)}$ centralizes one of the seven involutions in $C_{\text {Aut }(L)}(t) \cong \mathrm{D}_{12}$. Since there are four outer such involutions (including $t$ ) and three inner such involutions, part $(2)$ of $(*)$ follows.

Assume next that $u \in B_{2}$. Then every element at distance $\leq 3$ from $u$ in $\Delta_{\operatorname{Aut}(L)}$, centralizes one of the nine involutions in $C_{\mathrm{Aut}(L)}\left(u^{2}\right) \cong \mathrm{D}_{16}$. Since there are four outer such involutions and five inner such involutions, part (3) of $(*)$ follows. Now (*) implies that hypothesis (a) of Lemma [7.1.6 holds for all $\mathcal{B} \in\left\{B_{1}, B_{2}, B_{3}\right\}$ and $\mathcal{C} \in\left\{C_{1}, C_{2}, C_{3}\right\}$. It remains to show:

(**) Let $\mathcal{C}$ be the class of involutions of $L$. Then for each $j \in\{1,2,3\}$, if we set $\mathcal{B}=B_{j}$, then the pair $\mathcal{B}, \mathcal{C}$ satisfies hypothesis (b) of Lemma 7.1.6.

Indeed let $v \in \mathcal{C}$ be an involution and $1 \neq t \in C_{\text {Aut }(L)}(v)$. We will find $u^{\prime} \in \mathcal{B}$ such that (ii) holds ((i) appears in the proof of 7.3.1). Note again that we may assume that $t$ has prime order, so we may assume that $t$ is an involution. Since $C_{\text {Aut }(L)}(v) \cong \mathrm{D}_{16}$ has four outer involutions and five inner involutions, it follows that $\left|B_{1} \cap \Delta_{\operatorname{Aut}(L)}^{\leq 2}(v)\right|=8$ and that $\left|B_{2} \cap \Delta_{\operatorname{Aut}(L)}^{\leq 2}(v)\right|=10$. Also, if $t \notin L$, then $\left|B_{1} \cap \Delta_{\text {Aut }(L)}^{\leq 2}(t)\right|=8$ and $\left|B_{2} \cap \Delta_{\text {Aut }(L)}^{\leq 2}(t)\right|=6$. An easy counting argument now shows $(* *)$.

Lemma 7.3.3. Assume $L \cong \mathrm{A}_{6}$. Then $G$ has Property $\left(3 \frac{1}{2}\right)$.

Proof. Let $B_{1}, B_{2}, B_{3} \subseteq L$ be the $\operatorname{Aut}(L)$ conjugacy classes of elements of order $3,4,5$ and let $C_{1}, C_{2}, C_{3}, C_{4} \subseteq L$ be the $\operatorname{Aut}(L)$ conjugacy classes of elements of order $2,3,4,5$, respectively. We show that given $\mathcal{B} \in\left\{B_{1}, B_{2}, B_{3}\right\}$ and $\mathcal{C} \in\left\{C_{1}, \ldots, C_{4}\right\}$, hypothesis (b) or hypothesis (c) of Lemma 7.1.6 holds for $\mathcal{B}, \mathcal{C}$. Then, by Proposition 7.1.8(c), $G$ has Property $\left(3 \frac{1}{2}\right)$.

Write $B_{1}=B_{1}^{1} \cup B_{1}^{2}$, where $B_{1}^{1}$ is the $L$-class of 3 -cycles and $B_{1}^{2}$ is the other $L$-class of elements of order 3. Given $1 \neq v \in L$ and $t \in C_{\text {Aut }(L)}(v)$, we will find $u_{1}^{1} \in B_{1}^{1}, u_{1}^{2} \in B_{1}^{2}, u_{2} \in B_{2}$ and $u_{3} \in B_{3}$ (depending on $t$ ), such that each 
$u^{\prime} \in\left\{u_{1}^{1}, u_{1}^{2}, u_{2}, u_{3}\right\}$ satisfies the requirements in hypothesis (b) or (c) of Lemma 7.1.6. If $u^{\prime}$ satisfies the requirements in hypothesis (c) of Lemma 7.1.6 we will write $\pi\left(u^{\prime}, t\right)$ for the unique path of hypothesis (c), and we will indicate an $h^{\prime} \in L$ as required in hypothesis (c). We note now that given $1 \neq v \in L$, if we can show that for all $t \in C_{\operatorname{Aut}(L)}(v)$, there exists $u_{1}^{1}$ as above, and if, in addition, $C_{\operatorname{Aut}(L)}(v)$ contains an element interchanging $B_{1}^{1}$ and $B_{1}^{2}$, then we can also find $u_{1}^{2}$ as above. Note further that to establish hypothesis (b) or (c) of Lemma 7.1.6 we may assume without loss that the order of $t$ is a prime. Thus we distinguish the following cases.

CASE 1: $v=(12)(34)$.

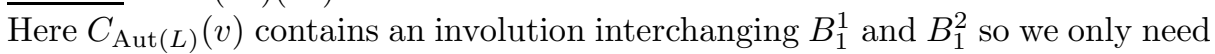
to establish the existence of $u_{1}^{1}, u_{2}$ and $u_{3}$. To simplify we denote $u_{1}=u_{1}^{1}$. Since $C_{\operatorname{Aut}(L)}(v)$ is a 2-group, we may assume that $t$ is an involution.

SUBCASE 1: $t \in L$.

We will show that hypothesis (b) holds in this case. If $t=(13)(24)$, take $u_{1}=$ (145), $u_{2}=(1346)(25)$ and $u_{3} \in B_{3}$ not inverted by $v$ or $t$. The case $t=(14)(23)$ is a conjugate case. If $t=(12)(56)$, take $u_{1}=(136), u_{2}=(1236)(45)$ and $u_{3} \in B_{3}$ not inverted by $v$ or $t$. The case $t=(34)(56)$ is a conjugate case.

SUBCASE 2: $t$ is a transposition or a product of three transpositions.

Suppose $t$ is a transposition. Then $t=(12),(34)$, or (56). Let $u_{1}=(135)$. We now list the path $\pi\left(u_{1}, t\right)$ and the element $h^{\prime}$, for the three possibilities of $t$.

$$
\begin{aligned}
& t=(12) \quad \pi\left(u_{1}, t\right)=(135), \quad(46), \quad(12) ; \quad h^{\prime}=(124) . \\
& t=(34) \quad \pi\left(u_{1}, t\right)=(135), \quad(26), \quad(34) ; \quad h^{\prime}=(124) . \\
& t=(56) \quad \pi\left(u_{1}, t\right)=(135), \quad(24), \quad(56) ; \quad h^{\prime}=(456) .
\end{aligned}
$$

We have $u_{1}^{h^{\prime}}=(135)^{(124)}\left(\right.$ resp. $\left.(135)^{(124)},(135)^{(456)}\right)$, so $u_{1}^{h^{\prime}}=(235)($ resp. $(235)$, (136)) and in all cases $u_{1}^{h^{\prime}} \in \Delta_{\operatorname{Aut}(L)}^{\geq 3}(v)$. Also, (46) $)^{(124)}=(16) \notin \Delta_{\operatorname{Aut}(L)}((12))$, $(26)^{(124)}=(46) \notin \Delta_{\operatorname{Aut}(L)}((34))$ and $(24)^{(456)}=(25) \notin \Delta_{\operatorname{Aut}(L)}((56))$. We get hypothesis (c).

Next let $u_{2}=(1235)(46)$ if $t=(34)$ or (56), and let $u_{2}=(1345)(26)$ if $t=(12)$. Finally, let $u_{3} \in B_{3}$ be an element not inverted by $v$. We get hypothesis (b).

Suppose now that $t$ is a product of three transpositions. If $t=(1 i)(2 j)(56)$, let $u_{1}=(1 i 5)$. Then $\pi\left(u_{1}, t\right)=u_{1},(2 j), t$. Then for $h^{\prime}=(2 j 6)$, we have $u_{1}^{h^{\prime}}=u_{1}$, $(2 j)^{h^{\prime}}=(2 j)^{(2 j 6)}=(j 6) \notin \Delta_{\text {Aut }(L)}(t)$. We get hypothesis (c) in this case. Next taking $u_{2}=(1 i 25)(j 6)$ and $u_{3} \in B_{3}$, we get hypothesis (b).

Suppose $t=(12)(34)(56)$. Let $u_{1}=(135)$. Then $\pi\left(u_{1}, t\right)=u_{1},(135)(246), t$. Then for $h^{\prime}=(124)$, we have $u_{1}^{h^{\prime}}=(135)^{(124)}=(235)$ and $((135)(246))^{(124)}=$ $(164)(235) \notin \Delta_{\operatorname{Aut}(L)}(t)$. We get hypothesis (c) in this case. Next taking $u_{2}=$ $(1235)(46)$ and $u_{3} \in B_{3}$, we get hypothesis (b).

SUBCASE $3: L\langle t\rangle \cong P G L(2,9)$.

We note that each element in $\Delta_{\text {Aut }(L)}^{\leq 2}(t)$ centralizes one of the 11 involutions in $C_{\operatorname{Aut}(L)}(t) \cong D_{20}$ and hence $B_{1} \cap \Delta_{\operatorname{Aut}(L)}^{\leq 2}(t)=\emptyset,\left|B_{2} \cap \Delta_{\operatorname{Aut}(L)}^{\leq 2}(t)\right|=10$ and $\left|B_{3} \cap \Delta_{\operatorname{Aut}(L)}^{\leq 2}(t)\right|=24$. Thus any $u_{1} \in B_{1}$ such that $d_{\text {Aut }(L)}\left(u_{1}, v\right) \geq 3$ will do. Next note that any two distinct elements $r, s \in B_{2} \cap \Delta_{\operatorname{Aut}(L)}^{\leq 2}(t)$ satisfy $\left\langle r^{2}, s^{2}\right\rangle \cong D_{10}$. So one of $u_{2}=(1235)(46)$ or $u_{2}=(1236)(45)$ is at distance $\geq 3$ from both $v$ and $t$ 
in $\Delta_{\text {Aut }(L)}$. Finally, any element $u_{3} \in B_{3} \backslash \Delta_{\operatorname{Aut}(L)}^{\leq 2}(t)$ which is not inverted by $v$ is at distance $\geq 3$ from both $v$ and $t$ in $\Delta_{\text {Aut }(L)}$. Hypothesis (b) holds in this case. CASE 2: $v=(123)$.

If $t=(456)$ (or $(465))$, let $u_{1}^{1}=(145)$. Then

$$
\pi\left(u_{1}^{1}, t\right)=(145), \quad(23), \quad(456) .
$$

We take $h^{\prime}=(126)$. Then $\left(u_{1}^{1}\right)^{h^{\prime}}=(145)^{(126)}=(245) \in \Delta_{\operatorname{Aut}(L)}^{\geq 3}(v)$ and $(23)^{h^{\prime}}=$ $(23)^{(126)}=(36) \notin \Delta_{\text {Aut }(L)}(456)$. We get hypothesis $(\mathrm{c})$.

Next we let $u_{1}^{2}=(124)(356), u_{2}=(2345)(16)$ and we take $u_{3} \in B_{3}$ to get hypothesis (b).

If $t=(123)(456)$, take $u_{1}^{1}=(145), u_{1}^{2}=(124)(365), u_{2}=(3456)(12)$ and take $u_{3} \in B_{3}$. We get hypothesis (b). The case when $t=(123)(465),(132)(456)$ or $(132)(465)$ are handled similarly.

If $t=(45)$, let $u_{1}^{1}=(346)$. Then

$$
\pi\left(u_{1}^{1}, t\right)=(346), \quad(12), \quad(45) .
$$

We take $h^{\prime}=(125)$. Then $\left(u_{1}^{1}\right)^{h^{\prime}}=u_{1}^{1}$ and $(12)^{h^{\prime}}=(12)^{(125)}=(25) \notin \Delta_{\text {Aut }(L)}((45))$. We get hypothesis (c).

Next we let $u_{1}^{2}=(124)(356), u_{2}=(2346)(15)$ and $u_{3} \in B_{3}$ to get hypothesis (b). The cases $t=(46)$ or $(56)$ are conjugate cases.

In cases 3 and 4 below, $C_{\operatorname{Aut}(L)}(v)$ contains an element interchanging $B_{1}^{1}$ and $B_{1}^{2}$ so we only need to establish the existence of $u_{1}^{1}, u_{2}$ and $u_{3}$. To simplify we denote $u_{1}=u_{1}^{1}$.

CASE 3: $v=(1234)(56)$

Assume that $t=(56)$. Let $u_{1}=(125)$. Then

$$
\pi\left(u_{1}, t\right)=(125), \quad(34), \quad(56) .
$$

We take $h^{\prime}=(356)$. Then $u_{1}^{h^{\prime}}=(125)^{(356)}=(126) \in \Delta_{\operatorname{Aut}(L)}^{\geq 3}(v)$ and $(34)^{h^{\prime}}=$ $(34)^{(356)}=(45) \notin \Delta_{\text {Aut }(L)}((56))$. We get hypothesis (c).

Next for $u_{2}=(2346)(15)$ and $u_{3} \in B_{3}$ we get hypothesis (b).

Assume that $t=(13)(24)(56)$ and let $u_{1}=(125)$. Then $\pi\left(u_{1}, t\right)=(125)$, $(125)(346), t$. We take $h^{\prime}=(123)$. Then $u_{1}^{h^{\prime}}=(125)^{(123)}=(235) \in \Delta_{\text {Aut }(L)}^{\geq 3}(v)$, and $((125)(346))^{(123)}=(146)(235) \notin \Delta_{\text {Aut }(L)}(t)$. We get hypothesis $(c)$.

Next for $u_{2}=(1325)(46)$ and $u_{3} \in B_{3}$ we get hypothesis (b).

Assume that $t=(13)(24)$. Then any $u_{i}$ at distance $\geq 3$ from $t$ in $\Delta_{\operatorname{Aut}(L)}$, $1 \leq i \leq 3$, will give us hypothesis (b).

CASE 4: $v=(12345)$.

Here $t$ is the unique (outer) involution in $C_{\text {Aut }(L)}(u)$. Take $u_{1} \in B_{1}$ (any such $\left.u_{1}\right), u_{2} \in B_{2}$ such that $u_{2}$ is not centralized by one of the five involutions in $C_{L}(t) \cong \mathrm{D}_{10}$. Finally, take $u_{3} \in B_{3}$ such that $u_{3}$ is not centralized by one of the six outer involutions in $C_{\operatorname{Aut}(L)}(t)$. Hypothesis (b) holds in this case.

Lemma 7.3.4. Let $L \cong P S L(3,4)$, and let $B$ be the conjugacy class of elements of $\operatorname{Aut}(L)$ of order 7 . Let $1 \neq w \in \operatorname{Aut}(L)$. Then

$$
\delta(w):=\left|\left\{u \in B \mid d_{\operatorname{Aut}(L)}(u, w) \leq 2\right\}\right|<\frac{1}{2}|B| .
$$

It follows that $G$ has Property $\left(3 \frac{1}{2}\right)$. 
Proof. By Corollary [7.1.9, to show that $G$ has Property $\left(3 \frac{1}{2}\right)$, it suffices to show that $\delta(w)<\frac{1}{2}|B|$, for all $1 \neq w \in \operatorname{Aut}(L)$, and that $L$ contains at least five distinct $\operatorname{Aut}(L)$ classes. Since $L$ contains the five $\operatorname{Aut}(L)$ classes $C_{1}, \ldots, C_{5}$ of elements of order $2,3,4,5,7$, respectively, it remains to show that $\delta(w)<\frac{1}{2}|B|$, for all $1 \neq w \in \operatorname{Aut}(L)$.

Note that we may assume that the order of $w$ is a prime number. Let $C$ be the conjugacy class of $w$ in $\operatorname{Aut}(L)$. Let $u \in B$, and recall that $C_{\operatorname{Aut}(L)}(u)=\langle u\rangle \times S_{u}$, with $S_{u} \cong S_{3}$. Let $t \in S_{u}$ be an involution and let $r \in S_{u}$ be an element of order 3 . Then $C_{\mathrm{Aut}(L)}(r) \cong 7: 6 \times 3$ and $C_{\operatorname{Aut}(L)}(r) \cap C_{\mathrm{Aut}(L)}(t) \cong 7: 6$. Since any element in $\Delta_{\operatorname{Aut}(L)}^{\leq 2}(u)$ centralizes some element of prime order in $C_{\operatorname{Aut}(L)}(u)$, we see that any element in $\Delta_{\operatorname{Aut}(L)}^{\leq 2}(u)$ whose order is a prime number either centralizes one of the three involutions in $S_{u}$ or is an outer automorphism of $L$ of order 3 and centralizes $r$. We use the notation of the ATLAS, pg. 24, for the conjugacy classes of elements in $\operatorname{Aut}(L)$.

If $C=5 A$, then $\delta(w)=0$, so assume that $C \neq 5 A$. The computations of $\delta(w)$ for $C=3 B$ or $3 C$ are slightly different and will be postponed to the end of the proof. To compute $\delta(w)$ we count the number of pairs

$$
\delta(C, B):=\left\{(u, w) \mid u \in B, w \in C \text { and } d_{\operatorname{Aut}(L)}(u, w) \leq 2\right\}
$$

in two ways. Let $t \in S_{u}$ be an involution and set $M=C_{\text {Aut }(L)}(t) \cong L_{3}(2): 2 \times 2$. As we noted, every $w \in C$ with $d_{\operatorname{Aut}(L)}(u, w) \leq 2$ centralizes one of the three involutions in $S_{u}$, hence we get that $\delta(C, B)$ is at most $3 \cdot|B| \cdot|M \cap C|$. On the other hand $\delta(C, B)=|C| \cdot \delta(w)$, so we see that

$$
\delta(w) \leq 3 \cdot|B| \cdot \frac{|M \cap C|}{|C|} .
$$

Hence we must show that

$$
\frac{|M \cap C|}{|C|}<\frac{1}{6}
$$

Since the number $\frac{|M|}{|\operatorname{Aut}(L)|}$ appears many times in our calculations, we note that $\frac{|M|}{\operatorname{Aut}(L) \mid}=\frac{1}{360}$. Table 1 summarizes our computations. We now explain our computations briefly, only in case we feel an explanation is needed. We note that the second column of the table gives the number of $M$-classes in $C \cap M$ and if it is more than one the third column gives the order of the possible centralizers.

TABLE 1.

\begin{tabular}{|c|c|c|c|c|}
\hline$C$ & & $\left|C_{M}(w)\right|$ & $\left|C_{\mathrm{Aut}(L)}(w)\right|$ & $\frac{|M \cap C|}{|C|}$ \\
\hline $2 A$ & 1 & $2^{5}$ & $2^{8} \cdot 3$ & $\frac{24}{360}$ \\
\hline $3 A$ & 1 & $2^{2} \cdot 3$ & $2^{2} \cdot 3^{3}$ & $\frac{9}{360}$ \\
\hline $7 A$ & 1 & 14 & 42 & $\frac{3}{360}$ \\
\hline $2 B$ & 1 & $2^{3} \cdot 3$ & $2^{5} \cdot 3^{3}$ & $\frac{36}{360}$ \\
\hline $2 C$ & 2 & $|M|, 2^{5}$ & $2^{5} \cdot 3 \cdot 7$ & $\frac{1}{360}+\frac{21}{360}=\frac{22}{360}$ \\
\hline $2 D$ & 1 & 24 & 240 & $\frac{10}{360}$ \\
\hline
\end{tabular}


Notice that in the cases $C=2 B, 2 D, C \cap M$ is contained in a subgroup of $M$ isomorphic to $L_{3}(2): 2$ and for $w \in C \cap M, w \notin L$. Hence $\left|C_{M}(w)\right|=\left|C_{M \cap L}(w)\right|$. $4=6 \cdot 4=24$. Also, for $C=2 C$, we have that $(C \cap M) \backslash\{t\}$ is contained in the subgroup $(M \cap L) \times\langle t\rangle$, so for $w \in(C \cap M) \backslash\{t\}$, we have $\left|C_{M}(w)\right|=2^{5}$.

Next assume that $C=3 B$ or $3 C$. We now must count the number of pairs $\delta(C, B)$ in a slightly different way. Given $u \in B$, we already saw that every $w \in C$ with $d_{\mathrm{Aut}(L)}(u, w) \leq 2$ centralizes the unique subgroup $\langle r\rangle$ of order 3 in $C_{\mathrm{Aut}(L)}(u)$. Now $C_{\text {Aut }(L)}(r) \cong 7: 6 \times 3$, so there are $7 \cdot 6+2=44$ elements of order 3 in $C_{\text {Aut }(L)}(r)$. Hence $\delta(C, B) \leq 44 \cdot|B|$ and also $\delta(C, B)=|C| \cdot \delta(w)$. So $\delta(w) \leq \frac{44}{|C|}|B|$ and we need to show that $\frac{44}{|C|}<\frac{1}{2}$. But $|C| \geq \frac{|\operatorname{Aut}(L)|}{60 \cdot 6}=\frac{2^{8} \cdot 3^{3} \cdot 5 \cdot 7}{2^{3} \cdot 3^{2} \cdot 5}=2^{5} \cdot 3 \cdot 7$, and hence $\frac{44}{|C|}<\frac{1}{2}$.

Lemma 7.3.5. Let $L \cong P S O^{+}(8,2)$, and let $B$ be the conjugacy class of elements of $\operatorname{Aut}(L)$ of order 7 . Let $C$ be any nonidentity conjugacy class of elements in $\operatorname{Aut}(L)$, and let $w \in C$. Then $\delta(w):=\left|\left\{u \in B \mid d_{\operatorname{Aut}(L)}(u, w) \leq 2\right\}\right| \leq \frac{3}{10}|B|$, unless either $C$ is the class of central involutions of $\operatorname{Aut}(L)$, or $C$ is a class of outer automorphisms, in which case $\delta(w) \leq \frac{3}{5}|B|$. It follows that $G$ has Property $\left(3 \frac{1}{2}\right)$.

Proof. Let $C_{1}, \ldots, C_{5} \subseteq L$ be any five distinct $\operatorname{Aut}(L)$ conjugacy classes such that $C_{i}$ is not the class of central involutions, for all $1 \leq i \leq 5$. Notice that once we prove the numerical bounds on $\delta(w)$, all the hypotheses of Corollary 7.1 .9 will be satisfied and so by Corollary 7.1.9 it will follow that $G$ has Property $\left(3 \frac{1}{2}\right)$.

The calculations here are very similar to those in the proof of Lemma 7.3.4. Here also for $u \in B$, we have $C_{\operatorname{Aut}(L)}(u)=\langle u\rangle \times S_{u}$, with $S_{u} \cong S_{3}$. The same assertions made in 7.3.4 hold here so we may consider only conjugacy classes $C$ such that the order of the elements in $C$ is a prime number. Let $t \in S_{u}$ be an involution and let $r \in S_{w}$ be an element of order 3 . Recall that $C_{\mathrm{Aut}(L)}(r) \cong G_{2}(2) \times 3$ and that $C_{\operatorname{Aut}(L)}(r) \cap C_{\operatorname{Aut}(L)}(t) \cong G_{2}(2)$. Again, as in the proof of Lemma 7.3.4, any element in $\Delta_{\operatorname{Aut}(L)}^{\leq 2}(u)$ whose order is a prime number either centralizes one of the three involutions in $S_{u}$ or is an outer automorphism of $L$ of order 3 and centralizes $r$. As in the proof of Lemma 7.3.4, this fact will be used in the calculations below.

We use the notation of the ATLAS, pg. 86, for the conjugacy classes of elements in $\operatorname{Aut}(L)$. We start by dealing with classes $C$ which are not outer automorphisms of order 3. Let $t \in S_{u}$ be an involution, and set $M=C_{\mathrm{Aut}(L)}(t) \cong P S p(6,2) \times 2$. As in the proof of Lemma 7.3.4 for $w \in C$, we have

$$
\delta(w) \leq \frac{3 \cdot|M \cap C|}{|C|} \cdot|B|,
$$

so we must compute $\frac{|M \cap C|}{|C|}$. As in the case of $P S L(3,4)$ we have $\frac{|M|}{|\operatorname{Aut}(L)|}=\frac{1}{360}$.

Table 2 summarizes our computations. The 5 -th column of Table 2 indicates to which class (or classes) of $P S p(6,2), C \cap M \cap L$ corresponds to. In the case of the outer automorphisms $\{2 F, 2 G\}$, the 5 -th column indicates the class of the projection of the involution to $P S p(6,2)$, in $P S p(6,2) \times 2$.

The case $C=2 B$ is the same as $C=2 C$ or $C=2 D$.

The case $C=3 A$ is the same as $C=3 B$ or $C=3 C$.

The case $C=5 A$ is the same as $C=5 B$ or $C=5 C$.

Next assume that $C=3 F$ or $3 G$. We now must count the number of pairs $\delta(C, B)$ in a slightly different way. Given $u \in B$, we already saw that every $w \in C$ 
TABLE 2.

\begin{tabular}{|c|c|c|c|c|c|}
\hline$C$ & & $\left|C_{M}(u)\right|$ & $\left|C_{\mathrm{Aut}(L)}(u)\right|$ & $C \cap M \cap L$ & $\frac{|M \cap C|}{|C|}$ \\
\hline $2 A$ & 1 & $2^{10} \cdot 3^{2}$ & $2^{13} \cdot 3^{4}$ & $2 B$ & $\frac{72}{360}$ \\
\hline $2 B$ & 1 & $2^{10} \cdot 3^{2} \cdot 5$ & $2^{11} \cdot 3^{2} \cdot 5$ & $2 A$ & $\frac{2}{360}$ \\
\hline $2 E$ & 2 & $2^{10} \cdot 3,2^{8} \cdot 3$ & $2^{11} \cdot 3^{2}$ & $2 C, 2 D$ & $\frac{6}{360}+\frac{24}{360}=\frac{30}{360}$ \\
\hline $3 A$ & 1 & $2^{5} \cdot 3^{3} \cdot 5$ & $2^{7} \cdot 3^{5} \cdot 5$ & $3 A$ & $\frac{36}{360}$ \\
\hline $3 D$ & 1 & $2^{4} \cdot 3^{4}$ & $2^{4} \cdot 3^{6}$ & $3 B$ & $\frac{9}{360}$ \\
\hline $3 E$ & 1 & $2^{3} \cdot 3^{3}$ & $2^{4} \cdot 3^{5}$ & $3 C$ & $\frac{18}{360}$ \\
\hline $5 A$ & 1 & $2^{2} \cdot 3 \cdot 5$ & $2^{3} \cdot 3 \cdot 5^{2}$ & $5 A$ & $\frac{10}{360}$ \\
\hline $7 A$ & 1 & 14 & 42 & $7 A$ & $\frac{3}{360}$ \\
\hline $2 F$ & 2 & $|M|, 2^{10} \cdot 3^{2} \cdot 5$ & $2 \cdot|P S p(6,2)|$ & $1 A, 2 A$ & $\frac{1}{360}+\frac{63}{360}=\frac{64}{360}$ \\
\hline $2 G$ & 3 & $2^{10} \cdot 3^{2}, 2^{10} \cdot 3,2^{8} \cdot 3$ & $2^{10} \cdot 3^{2}$ & $2 B, 2 C, 2 D$ & $\frac{1}{360}+\frac{3}{360}+\frac{12}{360}=\frac{16}{360}$ \\
\hline
\end{tabular}

with $d_{\operatorname{Aut}(L)}(u, w) \leq 2$ centralizes the unique subgroup $\langle r\rangle$ of order 3 in $C_{\operatorname{Aut}(L)}(u)$. Now $C_{\text {Aut }(L)}(r) \cong G_{2}(2) \times 3$. We first count the outer 3 -elements in this group. These have the form $r$ or $r^{2}$ times an element of order 1 or 3 in $G_{2}(2)^{\prime} \cong P S U(3,3)$. This group has 28 Sylow 3 -subgroups, each of order 27 . So $\delta(C, B) \leq 28 \cdot 27 \cdot 2 \cdot|B|$. Also $\delta(C, B)=|C| \cdot \delta(w)$, hence $\delta(w) \leq \frac{28 \cdot 27 \cdot 2}{|C|} \cdot|B|$. It suffices to show $\frac{28 \cdot 27 \cdot 2}{|C|}<\frac{3}{5}$, which is easy.

Lemma 7.3.6. Let $L \cong P S L(2,27)$. Then $G$ has Property $\left(3 \frac{1}{2}\right)$.

Proof. Let $B$ be the $\operatorname{Aut}(L)$ class of elements of order 13. We show that $\delta(w):=$ $\left|\left\{u \in B \mid d_{\operatorname{Aut}(L)}(u, w) \leq 2\right\}\right|<\frac{1}{2}|B|$, for all $1 \neq w \in \operatorname{Aut}(L)$. Since $L$ contains the five distinct $\operatorname{Aut}(L)$ conjugacy classes $C_{1}, \ldots, C_{5}$ of elements order $2,3,7,13$ and 14, respectively, Corollary 7.1.9 completes the proof.

Now given $u \in B$, we have $C_{\operatorname{Aut}(L)}(u)=\langle u\rangle\langle t\rangle$, where $t$ is an outer involution in $\operatorname{Aut}(L)$. It follows that $\Delta_{\operatorname{Aut}(L)}^{\leq 2}(u)=C_{\operatorname{Aut}(L)}(t) \backslash\{1\}$. Next we have that $M:=C_{\text {Aut }(L)}(t) \cong 26: 6$. Let $1 \neq w \in \operatorname{Aut}(L)$. The counting argument of Lemma 7.3 .4 shows that to prove that $\delta(w)<\frac{1}{2}|B|$, it suffices to show that $\frac{|M \cap C|}{|C|}<\frac{1}{2}$, where $C$ is the conjugacy class of $w$ in $\operatorname{Aut}(L)$. This is an easy calculation using the ATLAS (where, of course, we may assume that the order of $w$ is a prime number).

Lemma 7.3.7. Let $L \cong P S L(2,11)$ or $P S L(2,16)$. Then $G$ has Property $\left(3 \frac{1}{2}\right)$.

Proof. Again, as in the proof of Lemma 7.3.6 it suffices to show that there exists a conjugacy class $B$ of $L$ such that $\delta(w):=\left|\left\{u \in B \mid d_{\operatorname{Aut}(L)}(u, w) \leq 2\right\}\right|<\frac{1}{2}|B|$, for all $1 \neq w \in \operatorname{Aut}(L)$; and that $L$ contains at least five $\operatorname{Aut}(L)$ classes. For $P S L(2,11)$, let $B$ be the class of elements of order 11 , and for $P S L(2,16)$, let $B$ be the class of elements of order 17 . Since in both cases, given $u \in B$, we have $C_{\text {Aut }(L)}(u)=\langle u\rangle$, it is immediate that $\delta(w)<\frac{1}{2}|B|$, for all $1 \neq w \in \operatorname{Aut}(L)$. Also 
$L \cong P S L(2,11)$ has the $\operatorname{Aut}(L)$ classes of elements of order $2,3,5,6$ and 11 and $L \cong P S L(2,16)$ has the $\operatorname{Aut}(L)$ classes of elements of order 2, 3, 5, 15 and 17 , so the proof of the lemma is complete.

Lemma 7.3.8. Assume $L \cong \mathrm{A}_{5}$. Then $G$ has Property $\left(3 \frac{1}{2}\right)$.

Proof. Let $C_{1}, C_{2}, C_{3} \subseteq L$ be the $\operatorname{Aut}(L)$ conjugacy classes of elements of order $3,5,2$, respectively. We show that $L$ together with the classes $C_{1}, C_{2}, C_{3}$ satisfy all the hypotheses (a), (b) and (c) of Lemma 7.1.13.

Hypothesis (c): We must show that given $\mathcal{B}, \mathcal{C} \in\left\{C_{1}, C_{2}, C_{3}\right\}$, the pair $\mathcal{B}, \mathcal{C}$ satisfies hypothesis (c) of Lemma 7.1.13. For that we use Lemma 7.1.6. Given $\mathcal{C} \in\left\{C_{1}, \ldots, C_{3}\right\}, 1 \neq v \in \mathcal{C}$ and $t \in C_{\operatorname{Aut}(L)}(v)$, we will find $u_{1} \in C_{1}, u_{2} \in C_{2}$ and $u_{3} \in C_{3}$, such that each $u^{\prime} \in\left\{u_{1}, u_{2}, u_{3}\right\}$ satisfies the requirements in hypothesis (a), (b) or (c) of Lemma 7.1.6 Then, by Lemma [7.1.6, this will show that hypothesis (c) of Lemma 7.1.13 holds. If $u^{\prime}$ satisfies the requirements in hypothesis (c) of Lemma 7.1.6 we will write $\pi\left(u^{\prime}, t\right)$ for the unique path in hypothesis (c), and we will indicate an $h^{\prime} \in L$ as required in hypothesis (c2). Note that we may assume without loss that the order of $t$ is a prime.

When $\mathcal{C}=C_{3}$, we may assume without loss that $v=(12)(34)$. If

$$
v=(12)(34) \text { and } t=(13)(24) \text {, }
$$

take $u_{1}=(145), u_{2} \in C_{2}$ and $u_{3}=(14)(25)$. Here 7.1.6)(b) holds. The case $t=(14)(23)$ is a conjugate case. If

$$
v=(12)(34) \text { and } t=(12) \text {, }
$$

take $u_{1}=(235)$ and $u_{2} \in C_{2}$. Again $7.1 .6(\mathrm{~b})$ holds. For $u_{3}=(13)(25)$, we have

$$
\pi\left(u_{3}, t\right)=(13)(25), \quad(12)(35), \quad(12) .
$$

Let $h^{\prime}=(13)(24)$. Then $u_{3}^{h^{\prime}}=((13)(25))^{(13)(24)}=(13)(45) \in \Delta_{\operatorname{Aut}(L)}^{\geq 3}(v)$ and $(12)(35)^{(13)(24)}=(15)(34) \notin \Delta_{\text {Aut }(L)}((12))$. We get 7.1.6)(c). The case $t=(34)$ is a conjugate case.

When $\mathcal{C}=C_{1}$, we may assume that

$$
v=(123) \text { and } t=(45) \text {. }
$$

We take $u_{1}=(234)$ and $u_{2} \in C_{2}$. Hypothesis 7.1.6 b) holds. For $u_{3}=(14)(25)$, we have

$$
\pi\left(u_{3}, t\right)=(14)(25), \quad(12)(45), \quad(45) .
$$

Let $h^{\prime}=(245)$. Then $u_{3}^{h^{\prime}}=((14)(25))^{(245)}=(15)(24) \in \Delta_{\operatorname{Aut}(L)}^{\geq 3}(v)$ and $((12)(45))^{h^{\prime}}$ $=((12)(45))^{(245)}=(14)(25) \notin \Delta_{\operatorname{Aut}(L)}((45))$. We get 7.1.6(c). Finally hypothesis (a) of Lemma 7.1.6 clearly holds when $\mathcal{C}=C_{2}$, for any $\mathcal{B} \in\left\{C_{1}, C_{2}, C_{3}\right\}$.

Hypotheses (a) and (b): First we note that to show hypotheses (a) and (b) of Lemma 7.1.13, it is enough to show that they hold for some $v_{1} \in C_{1}$ and some $v_{2} \in C_{2}$. We thus start by picking

$$
v_{1}=(123) \quad \text { and } \quad v_{2}=(12345) .
$$

We now list the orbit representatives of $C_{\operatorname{Aut}(L)}\left(v_{l}\right)$ on $C_{j} \cap \Delta_{\operatorname{Aut}(L)}^{\geq 3}\left(v_{l}\right), l=1,2$ and $1 \leq j \leq 3$. We have $C_{\operatorname{Aut}(L)}\left(v_{1}\right)=\langle(123)\rangle \times\langle(45)\rangle$, and the orbit representatives are

$\{(124),(142),(145)\}, \quad\{(12345),(12435),(13245),(13425)\}, \quad\{(12)(34),(14)(25)\}$. 
We have $C_{\mathrm{Aut}(L)}\left(v_{2}\right)=\langle(12345)\rangle$, and the orbit representatives are

$$
\begin{gathered}
\{(123),(132),(124),(142)\}, \quad\{(12354),(12453),(12543),(13254)\}, \\
\{(12)(34),(13)(24),(14)(23)\} .
\end{gathered}
$$

We now show that hypothesis (b) of Lemma 7.1.13 holds for all possible choices of $j$ and $u$.

We start with $v=v_{1}$. For $j=1$, let $u \in C_{1}$ and let $h_{1}, h_{2} \in L$ such that $u^{h_{1}}=(124)$ and $u^{h_{2}}=(142)$. Then $d_{\operatorname{Aut}(L)}\left(u^{h_{i}}, v_{1}\right)>3$, for $i=1,2$, so for any orbit $\mathcal{O}$ of $C_{\operatorname{Aut}(L)}\left(v_{1}\right)$ on $C_{1} \cap \Delta_{\operatorname{Aut}(L)}^{\geq 3}\left(v_{1}\right)$, either $h=h_{1}$ or $h=h_{2}$ will satisfy all the requirements of hypothesis (b). Similarly, for $j=2$, it is enough to note that $d_{\text {Aut }(L)}\left(w, v_{1}\right)=\infty$, for all $w \in C_{2}$, and that for $u \in C_{2}, u^{L}$ meets at least two of the orbits of $C_{\mathrm{Aut}(L)}\left(v_{1}\right)$ on $C_{2} \cap \Delta_{\operatorname{Aut}(L)}^{\geq 3}\left(v_{1}\right)$.

Suppose $j=3$. Let $\mathcal{O}$ be an orbit of $C_{\operatorname{Aut}(L)}\left(v_{1}\right)$ on $C_{3} \cap \Delta_{\mathrm{Aut}(L)}^{\geq 3}\left(v_{1}\right)$. Let $u \in C_{3}$ be an involution. Let $s \in \Delta_{\operatorname{Aut}(L)}(u)$ and let $t \in \Delta_{\operatorname{Aut}(L)}\left(v_{1}\right)$. Suppose $(12)(34) \in \mathcal{O}$ and let $q \in L$, with $u^{q}=(14)(25)$. If $t \neq(45)$, or $s^{q} \neq(12)(45)$, then $\left[s^{q}, t\right] \neq 1$, so taking $h=q$, we see that hypothesis (b) holds. Thus $t=(45)$ and $s^{q}=(12)(45)$. Let $h=q(245)$. Then $u^{h}=((14)(25))^{(245)}=(15)(24)=((14)(25))^{(45)} \notin \mathcal{O}$ and $s^{h}=((12)(45))^{(245)}=(14)(25)$, so $\left[s^{h}, t\right] \neq 1$ and again hypothesis (b) holds. Next suppose that (14)(25) $\in \mathcal{O}$. Let $q \in L$, with $u^{q}=(12)(34)$. If $t \neq(45)$, or $s^{q} \neq(12)$, then $\left[s^{q}, t\right] \neq 1$, so taking $h=q$, we see that hypothesis (b) holds. Thus $t=(45)$ and $s^{q}=(12)$. Let $h=q(13)(24)$. Then $u^{h}=(12)(34) \notin \mathcal{O}$ and $s^{h}=(34)$, so $\left[s^{h}, t\right] \neq 1$ and again hypothesis (b) holds.

Suppose now that $v=v_{2}$. Note that for all $1 \leq j \leq 3$ and all $w \in \Delta^{>1}\left(v_{2}\right)$, $d_{\operatorname{Aut}(L)}\left(w, v_{2}\right)=\infty$. It follows that given $u \in C_{1} \cup C_{2} \cup C_{3}$, to show that hypothesis (b) holds it suffices to show that $u^{L}$ meets at least two orbits of $C_{\mathrm{Aut}(L)}\left(v_{2}\right)$ on $C_{j} \cap \Delta_{\operatorname{Aut}(L)}^{\geq 3}\left(v_{2}\right)$ and this is easy.

\section{REFERENCES}

[1] S. Amitsur, Finite subgroups of division rings, Trans. AMS 80(1955), 361-386. MR 17:577c

[2] M. Aschbacher, G.M. Seitz, Involutions in Chevalley groups over fields of even order, Nagoya Math. J. 63(1976), 1-91. MR 54:10391]

[3] J.H. Conway et al., ATLAS of finite groups, Clarendon Press, Oxford, 1985. MR 88g:20025

[4] H. Behr, Arithmetic groups over function fields. I. A complete characterization of finitely generated and finitely presented arithmetic subgroups of reductive algebraic groups, J. Reine Angew. Math. 495(1998), 79-118. MR 99g:20088

[5] N. Bourbaki, Algèbre commutative, Ch. V-VI, Masson, Paris, 1985. MR 86k:13001b

[6] V. Bergelson, D.B. Shapiro, Multiplicative subgroups of finite index in a ring, Proc. AMS 116(1992), 885-896. MR 93b:16001

[7] R.W. Carter, Conjugacy classes in the Weyl group, Compositio Math. 25(1972), 1-59. MR 47:6884

[8] - Finite Groups of Lie Type: Conjugacy Classes and Complex Characters, WileyInterscience, 1985. MR 87d:20060

[9] D. Gorenstein, R. Lyons, The local structure of finite groups of characteristic 2 type, Memoirs AMS 276 (1983), 1-731. MR 84g:20025

[10] S. Lang, Algebra, Addison-Wesley, 1965. MR 33:5416

[11] R. Lawther, M.W. Liebeck, G.M. Seitz, Fixed point ratios in actions of finite exceptional groups of Lie type, to appear, Pacific J. Math.

[12] M.W. Liebeck, G.M. Seitz, Reductive Subgroups of Exceptional Algebraic Groups, Memoirs AMS 580(1996), 1-111. MR 96i:20059

[13] G.A. Margulis, Finiteness of quotients of discrete groups, Funct. Analysis and Appl. 13(1979), 178-187. MR 80k:22006 
[14] R. Pierce, Associative Algebras, GTM 88, Springer, 1982. MR 84c:16001

[15] V.P. Platonov, A.S. Rapinchuk, Algebraic Groups and Number Theory, "Pure and Applied Mathematics" series, N 139, Academic Press, 1993. MR 95b:11039

[16] - The multiplicative structure of division algebras over number fields and the Hasse norm principle, Proc. Steklov Inst. Math. 165(1985), 187-205. MR 85j:11162

[17] G. Prasad, Strong approximation for semi-simple groups over function fields, Ann. Math. 105(1977), 553-572. MR 56:2921

[18] M.S. Raghunathan, On the group of norm 1 elements in a division algebra, Math. Ann. 279(1988), 457-484. MR 89g:11111

[19] A.S. Rapinchuk, A. Potapchik, Normal subgroups of $S L_{1, D}$ and the classification of finite simple groups, Proc. Indian Acad. Sci. 106(1996), 329-368. MR 98i:20049

[20] A.S. Rapinchuk, Y. Segev, Valuation-like maps and the congruence subgroup property, Invent. Math. 144(2001), 571-607. MR 2002e:16027

[21] L. Rowen, Y. Segev, The finite quotients of the multiplicative group of a division algebra of degree 3 are solvable, Israel J. of Math. 111(1999), 373-380. MR 2000g:16029

[22] - The multiplicative group of a division algebra of degree 5 and Wedderburn's Factorization Theorem, Contemp. Math. 259(2000), 475-486. MR 2001g:16037

[23] Y. Segev, On finite homomorphic images of the multiplicative group of a division algebra, Ann. Math. 149(1999), 219-251. MR 2000e:16022

[24] - Some applications of Wedderburn's factorization theorem, Bull. Austral. Math. Soc. 59(1999), 105-110. MR 99k:16068

[25] _ , The commuting graph of minimal nonsolvable groups, Geom. Ded. 88(2001), 5566.

[26] Y. Segev, G.M. Seitz, Anisotropic groups of type $A_{n}$ and the commuting graph of finite simple groups, Pacific J. Math. 202(2002), 125-226. CMP 2002:08

[27] A. Seress, The minimal base size of primitive solvable permutation groups, J. LMS (2) 53(1996), no. 2, 243-255. MR 96k:20003

[28] T.A. Springer, R. Steinberg, Conjugacy Classes, Springer Lecture Notes 131 (eds: A. Borel, et al.), Springer, Berlin, 1970. MR 42:3091

[29] R. Steinberg, Lectures on Chevalley Groups, Yale University Lecture Notes, 1967. MR 57:6215

[30] M. Suzuki, On a class of doubly transitive groups, Ann. Math. 75 (1962), 105-145. MR 25:112

[31] J. Tits, Algebraic and abstract simple groups, Ann. Math. 80(1964), no. 2, 313-329. MR 29:2259

[32] - Groupes de Whitehead de groupes algebriques simples sur un corps (d'apres V.P. Platonov et al.), Sem. Bourbaki, 1977, exp. 505. Lecture Notes in Math. 677(1978), 218-236. MR 80d:12008

[33] G. Turnwald, Multiplicative subgroups of finite index in rings, Proc. AMS 120(1994), 377381. MR 94e: 12002

[34] A.R. Wadsworth, Extending valuations to finite-dimensional division algebras, Proc. AMS 98(1986), 20-22. MR 87i:16025

Department of Mathematics, University of Virginia, Charlottesville, Virginia 22904

E-mail address: asr3x@weyl.math.virginia.edu

Department of Mathematics, Ben-Gurion University, Beer-Sheva 84105, Israel

E-mail address: yoavs@math.bgu.ac.il

Department of Mathematics, University of Oregon, Eugene, Oregon 97403-1226

E-mail address: seitz@math.uoregon.edu 